\title{
Ruthenium Catalyzed C-H Selenylations of Aryl Acetic Amides and Esters via Weak Coordination
}

Zhengyun Weng, Xinyue Fang, Meicui He, Linghui Gu, Jiafu Lin, Zheyu Li and Wenbo Ma* Antibiotics Research and Re-evaluation Key Laboratory of Sichuan Province, Sichuan Industrial Institute of Antibiotics, Chengdu University, P.R China, 610052. wenboma@ hotmail.com

General Remarks .52

Optimization of Catalytic Reaction Conditions...............................S3

Representative Procedure..................................................S4

Preparation and Characterization Data of Products.........................S5

Scale-up experiment of phenylacetamide 1i and diphenyl diselenide 2a...S26

Intermolecular Competition Experiment..................................S26

Ruthenium(II)-Catalyzed H/D Exchange Experiment......................S27

Control Experiment in the Presence of TEMPO..............................S28

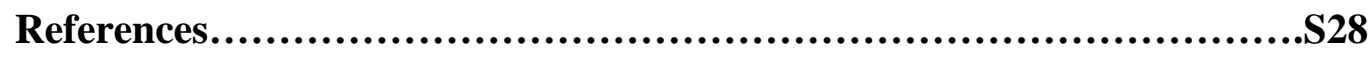

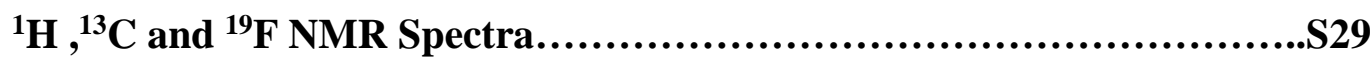




\section{General Remarks}

Catalytic reactions were carried out under argon. THF was distilled over Na. The starting materials were prepared according to previously described methods: ${ }^{[1]}$ Other chemicals were obtained from commercial sources, and were used without further purification. Yields refer to isolated compounds, estimated to be $>95 \%$ pure as determined by ${ }^{1}$ H-NMR. TLC: Macherey-Nagel, TLC plates Alugram R Sil G/UV254. Detection under UV light at $254 \mathrm{~nm}$. Chromatography: Separations were carried out on Merck Silica 60 (0.040-0.063 mm, 70-230 mesh ASTM). GC analysis was performed on Agilent 7890A GC-System. EI-MS: Finnigan MAT 95, 70eV; ESI-MS: Finnigan LCQ. High resolution mass spectrometry (HRMS): APEX IV 7T FTICR, Bruker Daltonic. M. p. Stuart R Melting Point Apparatus SMP3 melting point apparatus, values are uncorrected. ${ }^{1} \mathrm{H},{ }^{13} \mathrm{C},{ }^{19} \mathrm{~F}$-spectra were recorded at $600\left({ }^{1} \mathrm{H}\right), 150\left({ }^{13} \mathrm{C}\right)$ and ${ }^{19} \mathrm{~F}(565$ $\mathrm{MHz}$ ) respectively, on BRUKER instruments in $\mathrm{CDCl}_{3}$ solutions. If not otherwise specified, chemical shifts $(\delta)$ are given in ppm. 


\section{Optimization of Catalytic Reaction Conditions ${ }^{\mathrm{a}}$}

Table S-1:

\begin{tabular}{|c|c|c|c|c|}
\hline & & $\begin{array}{c}\text { PhSeSePh (2a) } \\
{\left[\mathrm{RuCl}_{2}(p \text {-cymene })\right]_{2}(5 \mathrm{~mol} \%} \\
\text { Additive }(20 \mathrm{~mol} \%) \\
\text { AgOPiv }(2.0 \text { equiv })^{\longrightarrow} \\
\mathrm{CF}_{3} \mathrm{SO}_{3} \mathrm{H}(1.0 \text { equiv }) \\
\text { Carboxylic acid (1.0 equiv) } \\
\text { TFE, } 100{ }^{\circ} \mathrm{C}, 16 \mathrm{~h}\end{array}$ & & \\
\hline Entry & Additive & Carboxylic Acid & Oxidant & Yield \\
\hline 1 & $\mathrm{AgBF}_{4}$ & --- & AgOPiv & 29 \\
\hline 2 & $\mathrm{AgBF}_{4}$ & HOAc & AgOPiv & 20 \\
\hline 3 & $\mathrm{AgBF}_{4}$ & $\mathrm{MesCO}_{2} \mathrm{H}$ & AgOPiv & trace \\
\hline 4 & $\mathrm{AgBF}_{4}$ & PivOH (10 mg) & AgOPiv & 29 \\
\hline 5 & $\mathrm{AgBF}_{4}$ & PivOH (25 mg) & AgOPiv & 38 \\
\hline 6 & $\mathrm{AgBF}_{4}$ & PivOH (40 mg) & AgOPiv & 32 \\
\hline 7 & $\mathrm{AgBF}_{4}$ & $\mathrm{AdmCO}_{2} \mathrm{H}(1.0 \mathrm{eq})$ & AgOPiv & 44 \\
\hline 8 & AgOTf & $\mathrm{AdmCO}_{2} \mathrm{H}(1.0 \mathrm{eq})$ & AgOPiv & 82 \\
\hline 9 & AgOTf & $\mathrm{AdmCO}_{2} \mathrm{H}(1.0 \mathrm{eq})$ & AgOPiv & $\operatorname{trace}^{\mathrm{b}}$ \\
\hline
\end{tabular}

${ }^{a}$ Reaction conditions: 1a (0.25 mmol), 2a (0.50 mmol), $\left[\mathrm{RuCl}_{2}(p \text {-cymene) }]_{2}(5 \mathrm{~mol} \%)\right.$, Additive (20 mol\%), $\mathrm{CF}_{3} \mathrm{SO}_{3} \mathrm{H}$ (1.0 equiv), solvent (2.0 mL), $100{ }^{\circ} \mathrm{C}, 16 \mathrm{~h}$, under Ar. ${ }^{b}$ without $\mathrm{CF}_{3} \mathrm{SO}_{3} \mathrm{H}$. 


\section{Representative Procedure}

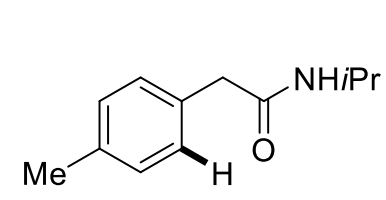

1

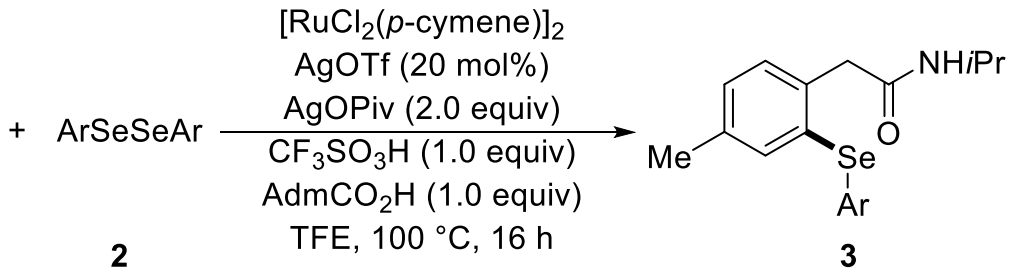

General Procedure A: Ruthenium-Catalyzed C-H Selenylation with $\mathrm{N}$-isopropyl2-(p-tolyl)acetamide A suspension of $N$-isopropyl-2-( $p$-tolyl) acetamide (1a) (1.0 equiv, $0.25 \mathrm{mmol}$ ), diaryl diselenide (2) (2.0 equiv, $0.50 \mathrm{mmol}),\left[\mathrm{RuCl}_{2}(p \text {-cymene })\right]_{2}$ (7.6 mg, $5.0 \mathrm{~mol} \%$ ), $\mathrm{CF}_{3} \mathrm{SO}_{3} \mathrm{H}$ (20 ul, $0.25 \mathrm{mmol}$ ), AgOTf (13 mg, $\left.20 \mathrm{~mol} \%\right)$, $\mathrm{AdmCO}_{2} \mathrm{H}(37.5 \mathrm{mg}, 0.25 \mathrm{mmol})$ and $\mathrm{AgOPiv}$ (103 mg 2.0 equiv) in $\mathrm{CF}_{3} \mathrm{CH}_{2} \mathrm{OH}$ (2.0 $\mathrm{mL}$ ) was stirred under argon at $100{ }^{\circ} \mathrm{C}$ for $16 \mathrm{~h}$. At ambient temperature, the reaction mixture was quench with $\mathrm{H}_{2} \mathrm{O}(10 \mathrm{~mL})$ and extracted with EtOAc $(3 \times 25 \mathrm{~mL})$. The combined organic layers were dried over anhydrous $\mathrm{Na}_{2} \mathrm{SO}_{4}$. After filtration and evaporation of the solvents in vacuo, the crude product was purified by column chromatography on silica gel (Petroleum/DCE: 2:1 $\rightarrow$ Petroleum/EtOAc: $4 / 1$ ) to afford the desired product $\mathbf{3}$.
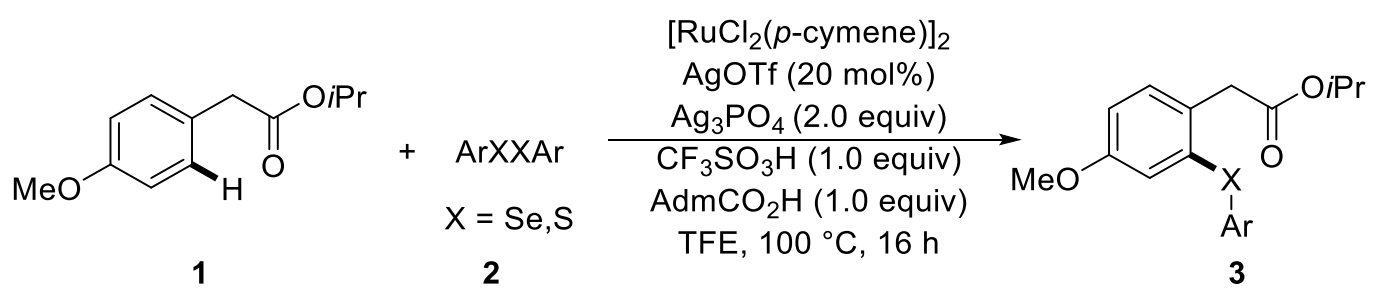

General Procedure B: Ruthenium-Catalyzed C-H Chalcogenation with Isopropyl

2-(4-methoxyphenyl)acetate A suspension of isopropyl 2-(4-methoxyphenyl)acetate (1) (1.0 equiv, $0.25 \mathrm{mmol}$ ), diaryl disulfide or diaryl diselenide (2) (2.0 equiv, 0.50 mmol), [RuCl${ }_{2}(p \text {-cymene) }]_{2}(7.6 \mathrm{mg}, 5.0 \mathrm{~mol} \%), \mathrm{CF}_{3} \mathrm{SO}_{3} \mathrm{H}$ (20 ul, $\left.0.25 \mathrm{mmol}\right)$, AgOTf (13 mg, $20 \mathrm{~mol} \%$ ), AdmCO ${ }_{2} \mathrm{H}\left(37.5 \mathrm{mg}, 0.25 \mathrm{mmol}\right.$ ) and $\mathrm{Ag}_{3} \mathrm{PO}_{4}$ (209 mg, 2.0 equiv) in $\mathrm{CF}_{3} \mathrm{CH}_{2} \mathrm{OH}(2.0 \mathrm{~mL})$ was stirred under argon at $100{ }^{\circ} \mathrm{C}$ for $16 \mathrm{~h}$. At ambient temperature, the reaction mixture was quench with $\mathrm{H}_{2} \mathrm{O}(10 \mathrm{~mL})$ and extracted with EtOAc ( 3 x $25 \mathrm{~mL}$ ). The combined organic layers were dried over anhydrous $\mathrm{Na}_{2} \mathrm{SO}_{4}$. After filtration and evaporation of the solvents in vacuo, the crude product was purified by column chromatography on silica gel (Petroleum /EtOAc: 10/1) to afford the desired product 3 . 
<smiles>Cc1ccc(CC(=O)NC(C)C)c([Se]c2ccccc2)c1</smiles>

$N$-isopropyl-2-(4-methyl-2-(phenylselanyl)phenyl)acetamide (3aa): The general procedure A was followed using $N$-isopropyl-2-( $p$-tolyl)acetamide (1a) (48 mg, 0.25 mmol) and 1,2-diphenyldiselane (2a) (156.0 mg, $0.5 \mathrm{mmol})$. Purification by column chromatography (Petroleum/DCE: $2 / 1 \rightarrow$ Petroleum/EtOAc: $4 / 1, \mathrm{Rf}=0.43$ ) yielded 3aa $(71 \mathrm{mg}, 82 \%)$ as a white solid. M.p $=90-92{ }^{\circ} \mathrm{C} .{ }^{1} \mathrm{H}$ NMR $(600 \mathrm{MHz}) \delta=7.35(\mathrm{~s}, 1 \mathrm{H})$, $7.30(\mathrm{~m}, 2 \mathrm{H}), 7.27(\mathrm{~d}, J=7.7 \mathrm{~Hz}, 1 \mathrm{H}), 7.24-7.21(\mathrm{~m}, 3 \mathrm{H}), 7.12(\mathrm{~d}, J=7.2 \mathrm{~Hz}, 1 \mathrm{H})$, $5.20(\mathrm{~s}, 1 \mathrm{H}), 4.09-3.86(\mathrm{~m}, 1 \mathrm{H}), 3.66(\mathrm{~s}, 2 \mathrm{H}), 2.27$ (s, 3H), 0.99 (d, J = 6.5 Hz, 6H). ${ }^{13} \mathrm{C} \mathrm{NMR}(150 \mathrm{MHz}) \delta=169.7,138.3,136.8,135.1,131.7,131.6,131.1,130.8,130.0$, $129.5,127.1,43.9,41.5,22.6,21.0$. HR-MS(ESI) $m / z$ calcd for: $\mathrm{C}_{18} \mathrm{H}_{22} \mathrm{NO}^{80} \mathrm{Se}^{+}\left[\mathrm{M}+\mathrm{H}^{+}\right]$ 348.0861 , found 348.0858 .<smiles>CCNC(=O)Cc1ccc(C)cc1[Se]c1ccccc1</smiles>

3ba

$N$-ethyl-2-(4-methyl-2-(phenylselanyl)phenyl)acetamide (3ba): The general procedure A was followed using $N$-ethyl-2-( $p$-tolyl)acetamide (1b) (45 mg, $0.25 \mathrm{mmol}$ ) and 1,2-diphenyldiselane (2a) (156.0 mg, $0.5 \mathrm{mmol})$. Purification by column chromatography (Petroleum/DCE: $2 / 1 \rightarrow$ Petroleum/EtOAc: $4 / 1, \mathrm{Rf}=0.43$ ) yielded $3 \mathrm{ba}$ $(70 \mathrm{mg}, 84 \%)$ as a white solid. M.p $=113-115{ }^{\circ} \mathrm{C} .{ }^{1} \mathrm{H}$ NMR $(600 \mathrm{MHz}) \delta=7.37$ (s, 1H), 7.31-7.28 (m, 2H), $7.28(\mathrm{~d}, J=7.7 \mathrm{~Hz}, 1 \mathrm{H}), 7.24-7.21(\mathrm{~m}, 3 \mathrm{H}), 7.14-7.12(\mathrm{~d}$, $J=8.0 \mathrm{~Hz}, 1 \mathrm{H}), 5.30(\mathrm{~s}, 1 \mathrm{H}), 3.69(\mathrm{~s}, 2 \mathrm{H}), 3.14-3.08(\mathrm{~m}, 2 \mathrm{H}), 2.28(\mathrm{~s}, 3 \mathrm{H}), 0.96(\mathrm{t}, J$ $=7.3 \mathrm{~Hz}, 3 \mathrm{H}) .{ }^{13} \mathrm{C}$ NMR $(151 \mathrm{MHz}) \delta=170.4,138.4,136.9,135.1,131.7,131.6,131.1$, 130.9, 130.1, 129.5, 127.1, 43.8, 34.6, 20.9, 14.7. HR-MS(ESI) $\mathrm{m} / z$ calcd for: $\mathrm{C}_{17} \mathrm{H}_{20} \mathrm{NO}^{80} \mathrm{Se}^{+}\left[\mathrm{M}+\mathrm{H}^{+}\right]$334.0705, found 334.0704. 
<smiles>Cc1ccc(CC(=O)NC2CCCCC2)c([Se]c2ccccc2)c1</smiles>

$N$-cyclohexyl-2-(4-methyl-2-(phenylselanyl)phenyl)acetamide (3ca): The general procedure A was followed using $N$-cyclohexyl-2-( $p$-tolyl)acetamide (1c) (57.8 mg, 0.25 mmol) and 1,2-diphenyldiselane (2a) (156.0 mg, $0.5 \mathrm{mmol})$. Purification by column chromatography (Petroleum/DCE: $2 / 1 \rightarrow$ Petroleum/EtOAc: $4 / 1, \mathrm{Rf}=0.30$ ) yielded 3ca $(75 \mathrm{mg}, 78 \%)$ as a white solid. M.p $=112-113{ }^{\circ} \mathrm{C} .{ }^{1} \mathrm{H}$ NMR $(600 \mathrm{MHz}) \delta=7.34$ (s, $1 \mathrm{H}), 7.30-7.27(\mathrm{~m}, 2 \mathrm{H}), 7.27(\mathrm{~d}, J=7.7 \mathrm{~Hz}, 1 \mathrm{H}), 7.25-7.21(\mathrm{~m}, 3 \mathrm{H}), 7.12(\mathrm{~d}, J=7.7$ $\mathrm{Hz}, 1 \mathrm{H}), 5.26(\mathrm{~s}, 1 \mathrm{H}), 3.72-3.67(\mathrm{~m}, 1 \mathrm{H}), 3.66(\mathrm{~s}, 2 \mathrm{H}), 2.27(\mathrm{~s}, 3 \mathrm{H}), 1.76-(\mathrm{m}, 3 \mathrm{H})$, $1.63-1.43(\mathrm{~m}, 2 \mathrm{H}), 1.32-1.22(\mathrm{~m}, 3), 1.13-1.02(\mathrm{~m}, 1 \mathrm{H}), 1.00-0.87(\mathrm{~m}, 2 \mathrm{H}) .{ }^{13} \mathrm{C}$ NMR $(150 \mathrm{MHz}) \delta=169.6,138.3,136.7,135.1,131.7,131.6,131.1,130.8,129.9$, 129.5, 127.1, 48.2, 43.9, 32.9, 25.6, 24.8, 21.0. HR-MS(ESI) $\mathrm{m} / \mathrm{z}$ calcd for: $\mathrm{C}_{21} \mathrm{H}_{26} \mathrm{NO}^{80} \mathrm{Se}^{+}\left[\mathrm{M}+\mathrm{H}^{+}\right]$388.1174, found 388.1174<smiles>Cc1ccc(CC(=O)NCC(C)C)c([Se]c2ccccc2)c1</smiles>

$3 d a$

$N$-isobutyl-2-(4-methyl-2-(phenylselanyl)phenyl)acetamide (3da) : The general procedure A was followed using $N$-isobutyl-2-( $p$-tolyl)acetamide (1d) (51.3 mg, 0.25 mmol) and 1,2-diphenyldiselane (2a) (156.0 mg, $0.5 \mathrm{mmol})$. Purification by column chromatography (Petroleum/DCE: $2 / 1 \rightarrow$ Petroleum/EtOAc: $4 / 1, \mathrm{Rf}=0.33$ ) yielded 3da $(65 \mathrm{mg}, 72 \%)$ as a white solid. M.p $=86-87{ }^{\circ} \mathrm{C} .{ }^{1} \mathrm{H}$ NMR $(600 \mathrm{MHz}) \delta=7.35$ (s, $1 \mathrm{H}), 7.31-7.29(\mathrm{~m}, 2 \mathrm{H}), 7.28(\mathrm{~d}, J=7.7 \mathrm{~Hz}, 1 \mathrm{H}), 7.24-7.22(\mathrm{~m}, 3 \mathrm{H}), 7.13$ (d, $J=$ $7.4 \mathrm{~Hz}, 1 \mathrm{H}), 5.40(\mathrm{~s}, 1 \mathrm{H}), 3.70(\mathrm{~s}, 2 \mathrm{H}), 2.91(\mathrm{~d}, J=6.5 \mathrm{~Hz}, 2 \mathrm{H}), 2.27(\mathrm{~s}, 3 \mathrm{H}), 1.60-$ $1.58(\mathrm{~m}, 1 \mathrm{H}), 0.78(\mathrm{~d}, J=6.7 \mathrm{~Hz}, 6 \mathrm{H}) .{ }^{13} \mathrm{C} \mathrm{NMR}(150 \mathrm{MHz}) \delta=170.6,138.4,136.7$, 135.0, 131.8, 131.4, 131.2, 130.9, 130.0, 129.5, 127.2, 47.0, 43.8, 28.5, 21.0, 20.1. HR-MS(ESI) $m / z$ calcd for: $\mathrm{C}_{19} \mathrm{H}_{24} \mathrm{NO}^{80} \mathrm{Se}^{+}\left[\mathrm{M}+\mathrm{H}^{+}\right] 362.1018$, found 362.1014 
<smiles>Cc1ccc(CC(=O)NC(C)C(=O)OC(C)C)c([Se]c2ccccc2)c1</smiles>

2-(4-methyl-2-(phenylselanyl)phenyl)-N'-(2-oxoethyl)acetohydrazide (3ea): The general procedure A was followed using $N^{\prime}$-(2-oxoethyl)-2-(p-tolyl)acetohydrazide (1e) (65.7 mg, $0.25 \mathrm{mmol}$ ) and 1,2-diphenyldiselane (2a) (156.0 mg, $0.5 \mathrm{mmol})$. Purification by column chromatography (Petroleum/DCE: $2 / 1 \rightarrow$ Petroleum/EtOAc: $4 / 1, \mathrm{Rf}=0.32$ ) yielded 3ea (75 mg, 72\%) as a white solid. M.p $=151-153{ }^{\circ} \mathrm{C} .{ }^{1} \mathrm{H}$ NMR $(600 \mathrm{MHz}) \delta$ $=7.38(\mathrm{~s}, 1 \mathrm{H}), 7.30-7.28(\mathrm{~m}, 2 \mathrm{H}), 7.28(\mathrm{~d}, J=7.4 \mathrm{~Hz}, 1 \mathrm{H}), 7.23-7.19(\mathrm{~m}, 3 \mathrm{H}), 7.14$ $(\mathrm{d}, J=7.5 \mathrm{~Hz}, 1 \mathrm{H}), 6.08(\mathrm{~s}, 1 \mathrm{H}), 5.02-4.94(\mathrm{~m}, 1 \mathrm{H}), 4.46-4.37(\mathrm{~m}, 1 \mathrm{H}), 3.72(\mathrm{~d}, J=$ $3.5 \mathrm{~Hz}, 2 \mathrm{H}), 2.27(\mathrm{~s}, 3 \mathrm{H}), 1.25(\mathrm{~d}, J=7.1 \mathrm{~Hz}, 3 \mathrm{H}), 1.21(\mathrm{~d}, J=6.2 \mathrm{~Hz}, 3 \mathrm{H}), 1.18(\mathrm{~d}, J$ $=6.2 \mathrm{~Hz}, 3 \mathrm{H}) .{ }^{13} \mathrm{C}$ NMR $(150 \mathrm{MHz}) \delta=172.4,170.1138 .5137 .0,134.8131 .8131 .6$ 131.1, 130.9130 .1129 .5127 .1 69.1, 48.4, 43.5, 21.8, 21.7 20.9, 18.4. HR-MS(ESI) $m / z$ calcd for: $\mathrm{C}_{21} \mathrm{H}_{26} \mathrm{NO}_{3}{ }^{80} \mathrm{Se}^{+}\left[\mathrm{M}+\mathrm{H}^{+}\right] 420.1072$, found 420.1071 .<smiles>Cc1ccc(CC(=O)N2CCCCC2)c([Se]c2ccc(Br)cc2)c1</smiles>

2-(4-methyl-2-(phenylselanyl)phenyl)-1-(piperidin-1-yl)ethan-1-one (3fa): The general procedure $\mathbf{A}$ was followed using 1-(piperidin-1-yl)-2-(p-tolyl)ethan-1-one (1f) (54.3mg, $0.25 \mathrm{mmol}$ ) and 1,2-diphenyldiselane (2a) (156.0 mg, $0.5 \mathrm{mmol})$. Purification by column chromatography (Petroleum/DCE: $2 / 1 \rightarrow$ Petroleum/EtOAc: $4 / 1, \mathrm{Rf}=0.30$ ) yielded 3fa (61 mg, 66\%) as a white solid. M.p $=101-103{ }^{\circ} \mathrm{C} .{ }^{1} \mathrm{H}$ NMR $(600 \mathrm{MHz}) \delta$ $=7.39(\mathrm{~s}, 1 \mathrm{H}), 7.30-7.28(\mathrm{~m}, 2 \mathrm{H}), 7.23-7.17(\mathrm{~m}, 4 \mathrm{H}), 7.11(\mathrm{~d}, J=7.4 \mathrm{~Hz}, 1 \mathrm{H}), 3.79$ $(\mathrm{s}, 2 \mathrm{H}), 3.55-3.50(\mathrm{~m}, 2 \mathrm{H}), 3.27-3.21(\mathrm{~m}, 2 \mathrm{H}), 2.27(\mathrm{~s}, 3 \mathrm{H}), 1.55-1.52(\mathrm{~m}, 2 \mathrm{H})$, 
$1.51-1.46(\mathrm{~m}, 2 \mathrm{H}), 1.33-1.31(\mathrm{~m}, 2 \mathrm{H}) .{ }^{13} \mathrm{C} \mathrm{NMR}(150 \mathrm{MHz}) \delta=169.4,137.6,136.7$, 135.7, 132.0, 131.3, 130.4, 129.8, 129.6, 129.4, 126.8, 47.1, 43.0, 40.6, 26.3, 25.6, 24.6, 20.9. HR-MS(ESI) $\mathrm{m} / z$ calcd for: $\mathrm{C}_{20} \mathrm{H}_{24} \mathrm{NO}^{80} \mathrm{Se}^{+}\left[\mathrm{M}+\mathrm{H}^{+}\right] 374.1018$, found 374.1015<smiles>NC(=O)Cc1ccccc1[Se]c1ccccc1</smiles>

3ga

2-(2-(phenylselanyl)phenyl)acetamide (3ga): The general procedure A was followed using 2-phenylacetamide (1g) (33.8 $\mathrm{mg}, 0.25 \mathrm{mmol})$ and 1,2-diphenyldiselane (2a) (156.0 mg, $0.5 \mathrm{mmol}$ ). Purification by column chromatography (Petroleum/DCE: $2 / 1 \rightarrow$ Petroleum/EtOAc: $1 / 1, \mathrm{Rf}=0.30)$ yielded $3 \mathrm{ga}(39 \mathrm{mg}, 54 \%)$ as a white solid. M.p $=109-110{ }^{\circ} \mathrm{C} .{ }^{1} \mathrm{H}$ NMR $(600 \mathrm{MHz}) \delta=7.49(\mathrm{~d}, J=8.1 \mathrm{~Hz}, 1 \mathrm{H}), 7.40(\mathrm{~d}, J=8.1$ $\mathrm{Hz}, 1 \mathrm{H}), 7.36-7.32(\mathrm{~m}, 2 \mathrm{H}), 7.33-7.29(\mathrm{~m}, 1 \mathrm{H}), 7.26-7.24(\mathrm{~m}, 3 \mathrm{H}), 7.19-7.17$ (m, $1 \mathrm{H}), 5.41(\mathrm{~s}, 2 \mathrm{H}), 3.76(\mathrm{~s}, 2 \mathrm{H}) .{ }^{13} \mathrm{C}$ NMR $(150 \mathrm{MHz}) \delta=172.7,137.5,135.8,132.4$, 132.1, 131.0, 129.6, 128.9, 128.7, 127.5, 43.5, 29.8. HR-MS(ESI) $\mathrm{m} / \mathrm{z}$ calcd for: $\mathrm{C}_{14} \mathrm{H}_{14} \mathrm{NO}^{80} \mathrm{Se}^{+}\left[\mathrm{M}+\mathrm{H}^{+}\right]$292.0235, found 292.0233.<smiles>CC(C)NC(=O)CCc1ccccc1[Se]c1ccccc1</smiles>

3ha

$N$-isopropyl-3-(2-(phenylselanyl)phenyl)propanamide(3ha): The general procedure A was followed using $N$-isopropyl-3-phenylpropanamide (1h) (48 $\mathrm{mg}, 0.25 \mathrm{mmol}$ ) and 1,2-diphenyldiselane (2a) (156.0 $\mathrm{mg}, \quad 0.5 \mathrm{mmol})$. Purification by column chromatography (Petroleum/DCE: $2 / 1 \rightarrow$ Petroleum/EtOAc: $4 / 1, \mathrm{Rf}=0.26$ ) yielded 3ha $(22 \mathrm{mg}, 25 \%)$ as a white solid. M.p $=143-145^{\circ} \mathrm{C} .{ }^{1} \mathrm{H}$ NMR $(600 \mathrm{MHz},) \delta=7.40(\mathrm{~d}, J$ $=7.8 \mathrm{~Hz}, 1 \mathrm{H}), 7.36-7.33(\mathrm{~m}, 2 \mathrm{H}), 7.28(\mathrm{dd}, J=7.6,1.1 \mathrm{~Hz}, 1 \mathrm{H}), 7.26-7.25(\mathrm{~m}, 3 \mathrm{H})$, 
$7.23(\mathrm{~d}, J=7.6 \mathrm{~Hz}, 1 \mathrm{H}), 7.10-7.08(\mathrm{~m}, 1 \mathrm{H}), 4.99(\mathrm{~s}, 1 \mathrm{H}), 3.99-3.97(\mathrm{~m}, 1 \mathrm{H}), 3.10$ (t, $J=7.6 \mathrm{~Hz}, 2 \mathrm{H}), 2.34(\mathrm{t}, J=8.1 \mathrm{~Hz}, 2 \mathrm{H}), 1.04(\mathrm{~d}, J=6.6 \mathrm{~Hz}, 6 \mathrm{H}) .{ }^{13} \mathrm{C} \mathrm{NMR}(151 \mathrm{MHz})$ $\delta=171.0,143.0,135.4,132.3,131.6,130.8,130.3,129.6,128.6,127.5,127.2$, 41.3, 38.0, 32.5, 22.8. HR-MS(ESI) $\mathrm{m} / z$ calcd for: $\mathrm{C}_{18} \mathrm{H}_{22} \mathrm{NO}^{80} \mathrm{Se}^{+}\left[\mathrm{M}+\mathrm{H}^{+}\right] 348.0861$, found 348.0854 .<smiles>Cc1ccc([Se]c2ccccc2)c(CC(=O)NC(C)C)c1</smiles>

$\mathrm{N}$-isopropyl-2-(5-methyl-2-(phenylselanyl)phenyl)acetamide (3ia): The general procedure A was followed using $N$-isopropyl-2-( $m$-tolyl)acetamide (1i) (48 mg, 0.25 mmol) and 1,2-diphenyldiselane (2a) $(156.0 \mathrm{mg}, 0.5 \mathrm{mmol})$. Purification by column chromatography (Petroleum/DCE: $2 / 1 \rightarrow$ Petroleum/EtOAc: $4 / 1, \mathrm{Rf}=0.41$ ) yielded 3ia (64 mg, 74\%) as a white solid. M.p $=96-97{ }^{\circ} \mathrm{C} .{ }^{1} \mathrm{H}$ NMR $(600 \mathrm{MHz}) \delta=7.45(\mathrm{~d}, J=$ $7.8 \mathrm{~Hz}, 1 \mathrm{H}), 7.29-7.26(\mathrm{~m}, 2 \mathrm{H}), 7.23(\mathrm{~s}, 1 \mathrm{H}), 7.22-7.18(\mathrm{~m}, 3 \mathrm{H}), 7.02(\mathrm{~d}, J=7.5 \mathrm{~Hz}$ 1H), $5.47(\mathrm{~s}, 1 \mathrm{H}), 4.00-3.98(\mathrm{~m}, 1 \mathrm{H}), 3.66(\mathrm{~s}, 2 \mathrm{H}), 2.33(\mathrm{~s}, 3 \mathrm{H}), 0.98(\mathrm{~d}, J=6.7 \mathrm{~Hz}$, $6 \mathrm{H}) .{ }^{13} \mathrm{C} \mathrm{NMR}(150 \mathrm{MHz}) \delta=169.6,139.5,138.4,136.8,132.2,131.9,131.1,129.5$, 129.4, 127.4, 126.9, 44.3, 41.6, 22.6, 21.2. HR-MS(ESI) $\mathrm{m} / \mathrm{z}$ calcd for: $\mathrm{C}_{18} \mathrm{H}_{21} \mathrm{NONa}^{80} \mathrm{Se}^{+}\left[\mathrm{M}+\mathrm{Na}^{+}\right] 370.0681$, found 370.0680 .<smiles></smiles>

$\mathrm{N}$-isopropyl-2-(5-methoxy-2-(phenylselanyl)phenyl)acetamide (3ja): The general procedure A was followed using $N$-isopropyl-2-(3-methoxyphenyl)acetamide (1j) (51.8 $\mathrm{mg}, 0.25 \mathrm{mmol}$ ) and 1,2-diphenyldiselane (2a) (156.0 mg, $0.5 \mathrm{mmol})$. Purification by column chromatography (Petroleum/DCE: $2 / 1 \rightarrow$ Petroleum/EtOAc: $4 / 1, \mathrm{Rf}=0.43$ ) yielded 3ja (65 mg, 72\%) as a white solid. M.p $=94-96{ }^{\circ} \mathrm{C} .{ }^{1} \mathrm{H}$ NMR $(600 \mathrm{MHz}) \delta=$ 
$7.61(\mathrm{~d}, J=8.3 \mathrm{~Hz} 1 \mathrm{H}), 7.19-7.16(\mathrm{~m}, 5 \mathrm{H}), 7.00(\mathrm{~d}, J=2.8 \mathrm{~Hz}, 1 \mathrm{H}), 6.78(\mathrm{dd}, J=8.3$, $2.8 \mathrm{~Hz}, 1 \mathrm{H}), 5.22(\mathrm{~s}, 1 \mathrm{H}), 4.04-3.87(\mathrm{~m}, 1 \mathrm{H}), 3.81(\mathrm{~s}, 3 \mathrm{H}), 3.68(\mathrm{~s}, 2 \mathrm{H}), 0.95$ (d, $J=$ $6.4 \mathrm{~Hz}, 6 \mathrm{H}) .{ }^{13} \mathrm{C}$ NMR $(150 \mathrm{MHz}) \delta=169.5,160.7,140.9,139.3,133.1,130.0,129.5$, 126.5, 120.6, 116.3, 114.6, 55.5, 44.7, 41.6, 22.5. HR-MS(ESI) $\mathrm{m} / \mathrm{z}$ calcd for: $\mathrm{C}_{18} \mathrm{H}_{21} \mathrm{NO}_{2} \mathrm{Na}^{80} \mathrm{Se}^{+}\left[\mathrm{M}+\mathrm{Na}^{+}\right]$386.0630, found 386.0632.<smiles>Cc1cc(CC(=O)NC(C)C)c([Se]c2ccccc2)cc1C</smiles>

$3 \mathrm{ka}$

2-(4,5-dimethyl-2-(phenylselanyl)phenyl)- $N$-isopropylacetamide $\quad$ (3ka): The general procedure A was followed using 2-(3,4-dimethylphenyl)-isopropylacetamide (1k) (51.3 mg, $0.25 \mathrm{mmol})$ and 1,2-diphenyldiselane (2a) (156.0 mg, $0.5 \mathrm{mmol})$. Purification by column chromatography (Petroleum/DCE: 2/1 $\rightarrow$ Petroleum/EtOAc: 4/1, $\mathrm{Rf}=0.36)$ yielded $3 \mathbf{k a}(59 \mathrm{mg}, 66 \%)$ as a white solid. M.p $=124-126{ }^{\circ} \mathrm{C} .{ }^{1} \mathrm{H}$ NMR $(600 \mathrm{MHz}) \delta=7.37(\mathrm{~s}, 1 \mathrm{H}), 7.25(\mathrm{~s}, 1 \mathrm{H}), 7.24-7.22(\mathrm{~m}, 1 \mathrm{H}), 7.22-7.17(\mathrm{~m}, 4 \mathrm{H}), 5.41$ (s, $1 \mathrm{H}), 4.00-3.89(\mathrm{~m}, 1 \mathrm{H}), 3.63$ (s, 2H), 2.24 (s, 3H), 2.19 (s, 3H), 0.97 (d, J = 6.5 $\mathrm{Hz}, 6 \mathrm{H}) .{ }^{13} \mathrm{C}$ NMR $(150 \mathrm{MHz}) \delta=169.9,138.4,138.2,137.1,136.1,132.5,132.2$, 130.7, 129.5, 127.1, 126.7, 43.9, 41.5, 22.6, 19.6, 19.3. HR-MS(ESI) $\mathrm{m} / z$ calcd for: $\mathrm{C}_{19} \mathrm{H}_{24} \mathrm{NO}^{80} \mathrm{Se}^{+}\left[\mathrm{M}+\mathrm{H}^{+}\right] 362.1018$, found 362.1017<smiles>COc1cc(CC(=O)NC(C)C)c([Se]c2ccccc2)cc1OC</smiles>

2-(4,5-dimethoxy-2-(phenylselanyl)phenyl)- $N$-isopropylacetamide (3la): The general procedure A was followed using 2-(3,4-dimethoxyphenyl)- $N$ isopropylacetamide (11) (59.3 mg, $0.25 \mathrm{mmol}$ ) and 1,2-diphenyldiselane (2a) (156.0 mg, $0.5 \mathrm{mmol}$ ). Purification by column chromatography (Petroleum/DCE: $2 / 1 \rightarrow$ Petroleum/EtOAc: $4 / 1, \mathrm{Rf}=0.35)$ yielded 3la $(77 \mathrm{mg}, 79 \%)$ as a white solid. $\mathrm{M} . \mathrm{p}=$ 128-130 ${ }^{\circ} \mathrm{C} .{ }^{1} \mathrm{H}$ NMR $(600 \mathrm{MHz}) \delta=7.22-7.15(\mathrm{~m}, 5 \mathrm{H}), 7.13(\mathrm{~s}, 1 \mathrm{H}), 6.97(\mathrm{~s}, 1 \mathrm{H})$, $5.19(\mathrm{~s}, 1 \mathrm{H}), 3.96-3.90(\mathrm{~m}, 1 \mathrm{H}), 3.89(\mathrm{~s}, 3 \mathrm{H}), 3.81(\mathrm{~s}, 3 \mathrm{H}), 3.65(\mathrm{~s}, 2 \mathrm{H}), 0.94(\mathrm{~d}, J=$ 
$6.7 \mathrm{~Hz}, 6 \mathrm{H}) .{ }^{13} \mathrm{C}$ NMR $(150 \mathrm{MHz}) \delta=169.9,150.1,148.4,133.0,132.3,129.9,129.5$, 126.6, 120.1, 120.0, 113.3, 56.2, 56.1, 44.1, 41.5, 22.5. HR-MS(ESI) $\mathrm{m} / \mathrm{z}$ calcd for: $\mathrm{C}_{19} \mathrm{H}_{23} \mathrm{NO}_{3} \mathrm{Na}^{80} \mathrm{Se}^{+}\left[\mathrm{M}+\mathrm{Na}^{+}\right] 416.0735$, found 416.0737<smiles>CCCNC(=O)Cc1cc2c(cc1[Se]c1ccccc1)OCO2</smiles>

$N$-isopropyl-2-(6-(phenylselanyl)benzo[d][1,3]dioxol-5-yl)acetamide (3ma): The general procedure $\mathbf{A}$ was followed using 2-(benzo[ $d][1,3]$ dioxol-5-yl)- $N$ isopropylacetamide (1 $\mathbf{m})(55.3 \mathrm{mg}, 0.25 \mathrm{mmol})$ and 1,2-diphenyldiselane (2a) (156.0 $\mathrm{mg}, 0.5 \mathrm{mmol}$ ). Purification by column chromatography (Petroleum/DCE: $2 / 1 \rightarrow$ Petroleum/EtOAc: $4 / 1, \mathrm{Rf}=0.35)$ yielded $3 \mathrm{ma}(72 \mathrm{mg}, 77 \%)$ as a white solid. M.p = 133-134 ${ }^{\circ} \mathrm{C} .{ }^{1} \mathrm{H}$ NMR $(600 \mathrm{MHz}) \delta=7.25-7.23(\mathrm{~m}, 2 \mathrm{H}), 7.23-7.18(\mathrm{~m}, 3 \mathrm{H}), 7.04$ $(\mathrm{s}, 1 \mathrm{H}), 6.92(\mathrm{~s}, 1 \mathrm{H}), 5.97(\mathrm{~s}, 2 \mathrm{H}), 5.23(\mathrm{~s}, 1 \mathrm{H}), 4.00-3.91(\mathrm{~m}, 1 \mathrm{H}), 3.64(\mathrm{~s}, 2 \mathrm{H}), 0.98$ $(\mathrm{d}, J=6.4 \mathrm{~Hz}, 6 \mathrm{H}) .{ }^{13} \mathrm{C}$ NMR $(150 \mathrm{MHz}) \delta=169.6,148.9,147.5,132.7,132.5,130.8$, 129.6, 126.9, 121.9, 116.5, 110.8, 101.7, 44.2, 41.6, 22.6. HR-MS(ESI) $m / z$ calcd for: $\mathrm{C}_{18} \mathrm{H}_{19} \mathrm{NO}_{3} \mathrm{Na}^{80} \mathrm{Se}^{+}\left[\mathrm{M}+\mathrm{Na}^{+}\right] 400.0422$, found 400.0426 .<smiles>CC(C)NC(=O)Cc1ccccc1Oc1ccccc1</smiles>

$N$-isopropyl-2-(2-(phenylselanyl)phenyl)acetamide (3na): The general procedure A was followed using $N$-isopropyl-2-phenylacetamide (1n) $(44.5 \mathrm{mg}, 0.25 \mathrm{mmol})$ and 1,2-diphenyldiselane (2a) (156.0 $\mathrm{mg}, \quad 0.5 \mathrm{mmol})$. Purification by column chromatography (Petroleum/DCE: $2 / 1 \rightarrow$ Petroleum/EtOAc: $4 / 1, \mathrm{Rf}=0.33$ ) yielded 3na (36 mg, 44\%) as a white solid. M.p = 109-111 ${ }^{\circ} \mathrm{C} .{ }^{1} \mathrm{H}$ NMR $(600 \mathrm{MHz}) \delta=7.48$ $(\mathrm{d}, J=7.9 \mathrm{~Hz}, 1 \mathrm{H}), 7.38(\mathrm{~d}, J=7.6 \mathrm{~Hz}, 1 \mathrm{H}), 7.33-7.30(\mathrm{~m}, 2 \mathrm{H}), 7.30(\mathrm{~m}, 1 \mathrm{H}), 7.26-$ $7.22(\mathrm{~m}, 3 \mathrm{H}), 7.17-7.15(\mathrm{~m}, 1 \mathrm{H}), 5.26(\mathrm{~s}, 1 \mathrm{H}), 4.04-3.94(\mathrm{~m}, 1 \mathrm{H}), 3.70(\mathrm{~s}, 2 \mathrm{H}), 1.00$ 
$(\mathrm{d}, J=6.7 \mathrm{~Hz}, 6 \mathrm{H}) .{ }^{13} \mathrm{C} \mathrm{NMR}(150 \mathrm{MHz}) \delta=169.4,137.9,135.8,132.1,131.9,131.3$, 131.0, 129.6, 128.9, 128.4, 127.4, 44.3, 41.6, 22.6. HR-MS(ESI) $\mathrm{m} / z$ calcd for: $\mathrm{C}_{17} \mathrm{H}_{20} \mathrm{NO}^{80} \mathrm{Se}^{+}\left[\mathrm{M}+\mathrm{H}^{+}\right] 334.0705$, found 334.0701<smiles>CC(C)NC(=O)Cc1ccc(C(C)(C)C)cc1[Se]c1ccccc1</smiles>

3oa

2-(4-(tert-butyl)-2-(phenylselanyl)phenyl)- $N$-isopropylacetamide $\quad$ (3oa): $\quad$ The general procedure $\mathbf{A}$ was followed using 2-(4-(tert-butyl)phenyl)- $N$ isopropylacetamide (1o) $(58.3 \mathrm{mg}, 0.25 \mathrm{mmol})$ and 1,2-diphenyldiselane (2a) (156.0 $\mathrm{mg}, 0.5 \mathrm{mmol}$ ). Purification by column chromatography (Petroleum/DCE: $2 / 1 \rightarrow$ Petroleum/EtOAc: $4 / 1, \mathrm{Rf}=0.36)$ yielded 3oa $(40 \mathrm{mg}, 41 \%)$ as a white solid. M.p = $112-114{ }^{\circ} \mathrm{C} . \quad{ }^{1} \mathrm{H}$ NMR $(600 \mathrm{MHz}) \delta=7.54(\mathrm{~d}, J=1.0 \mathrm{~Hz}, 1 \mathrm{H}), 7.33-7.30(\mathrm{~m}, 2 \mathrm{H})$, $7.29-7.27(\mathrm{~m}, 2 \mathrm{H}), 7.22-7.18(\mathrm{~m}, 3 \mathrm{H}), 5.25(\mathrm{~s}, 1 \mathrm{H}), 4.03-3.98(\mathrm{~m}, 1 \mathrm{H}), 3.65(\mathrm{~s}$, $2 \mathrm{H}), 1.24(\mathrm{~s}, 9 \mathrm{H}), 0.99(\mathrm{~d}, J=6.5 \mathrm{~Hz}, 6 \mathrm{H}) .{ }^{13} \mathrm{C} \mathrm{NMR}(150 \mathrm{MHz}) \delta=169.7,151.5$, 135.2, 133.5, 131.9, 131.4, 130.7, 130.6, 129.5, 127.0, 126.3, 43.8, 41.6, 34.7, 31.2, 22.6. HR-MS(ESI) $\mathrm{m} / z$ calcd for: $\mathrm{C}_{21} \mathrm{H}_{27} \mathrm{NONa}^{80} \mathrm{Se}^{+}\left[\mathrm{M}+\mathrm{Na}^{+}\right] 412.1150$, found 412.1153<smiles>CCCNC(=O)Cc1ccc(Br)cc1[Se]c1ccc(Br)cc1</smiles>

2-(4-bromo-2-(phenylselanyl)phenyl)- $N$-isopropylacetamide (3pa): The general procedure A was followed using 2-(4-bromophenyl)- $N$-isopropylacetamide (1p) (63.8 $\mathrm{mg}, 0.25 \mathrm{mmol}$ ) and 1,2-diphenyldiselane (2a) (156.0 $\mathrm{mg}, 0.5 \mathrm{mmol})$. Purification by column chromatography (Petroleum/DCE: $2 / 1 \rightarrow$ Petroleum/EtOAc: $4 / 1, \mathrm{Rf}=0.32$ ) yielded 3pa (41 mg, 40\%) as a white solid. M.p = 112-114 ${ }^{\circ} \mathrm{C} .{ }^{1} \mathrm{H}$ NMR $(600 \mathrm{MHz}) \delta$ $=7.53(\mathrm{~d}, J=2.0 \mathrm{~Hz}, 1 \mathrm{H}), 7.40(\mathrm{dd}, J=8.2,2.0 \mathrm{~Hz}, 1 \mathrm{H}), 7.38-7.36(\mathrm{~m}, 2 \mathrm{H}), 7.31-$ $7.27(\mathrm{~m}, 3 \mathrm{H}), 7.24(\mathrm{~d}, J=8.2 \mathrm{~Hz}, 1 \mathrm{H}), 5.21(\mathrm{~s}, 1 \mathrm{H}), 4.08-3.92(\mathrm{~m}, 1 \mathrm{H}), 3.63(\mathrm{~s}, 2 \mathrm{H})$, $1.03(\mathrm{~d}, J=6.7 \mathrm{~Hz}, 6 \mathrm{H}) .{ }^{13} \mathrm{C} \mathrm{NMR}(150 \mathrm{MHz}) \delta=168.8,137.1,136.2,134.3,132.8$, 
132.3, 131.6, 130.2, 129.9, 128.0, 121.9, 43.5, 41.7, 22.6. HR-MS(ESI) $m / z$ calcd for: $\mathrm{C}_{17} \mathrm{H}_{19} \mathrm{BrNO}^{80} \mathrm{Se}^{+}\left[\mathrm{M}+\mathrm{H}^{+}\right]$411.9810, found 411.9803<smiles>CCCNC(=O)Cc1cc(F)cc(F)c1[Se]c1ccccc1</smiles>

2-(3,5-difluoro-2-(phenylselanyl)phenyl)- $N$-isopropylacetamide (3qa): The general procedure A was followed using 2-(3,5-difluorophenyl)- $N$-isopropylacetamide (1q) (53.3 $\mathrm{mg}, 0.25 \mathrm{mmol}$ ) and 1,2-diphenyldiselane (2a) (156.0 mg, $0.5 \mathrm{mmol})$. Purification by column chromatography (Petroleum/DCE: $2 / 1 \rightarrow$ Petroleum/EtOAc: $4 / 1, \mathrm{Rf}=0.36$ ) yielded 3qa (51 mg, 55\%) as a white solid. M.p $=92-93{ }^{\circ} \mathrm{C} .{ }^{1} \mathrm{H}$ NMR $(600 \mathrm{MHz}) \delta=$ $7.24-7.17(\mathrm{~m}, 5 \mathrm{H}), 7.07-7.02(\mathrm{~m}, 1 \mathrm{H}), 6.83-6.81(\mathrm{~m}, 1 \mathrm{H}), 5.26(\mathrm{~s}, 1 \mathrm{H}), 3.98-3.88$ $(\mathrm{m}, 1 \mathrm{H}), 3.77(\mathrm{~s}, 2 \mathrm{H}), 0.99(\mathrm{~d}, J=6.6 \mathrm{~Hz}, 6 \mathrm{H}) .{ }^{13} \mathrm{C} \mathrm{NMR}(150 \mathrm{MHz}) \delta=168.4,163.8$ $(\mathrm{dd}, J=252.1,11.9 \mathrm{~Hz}), 163.7(\mathrm{dd}, J=246.3,8.6 \mathrm{~Hz}), 143.7(\mathrm{~d}, J=9.1 \mathrm{~Hz}), 131.6$, 129.9, 129.3, 127.0, $113.9(\mathrm{dd}, J=21.9,3.2 \mathrm{~Hz}), 113.0(\mathrm{dd}, J=22.4,3.7 \mathrm{~Hz}), 103.5$ (dd, $J=29.2,25.4 \mathrm{~Hz}$ ), 44.2, 41.8, 22.5. HR-MS(ESI) $m / z$ calcd for: $\mathrm{C}_{17} \mathrm{H}_{18} \mathrm{~F}_{2} \mathrm{NO}^{80} \mathrm{Se}^{+}$ $\left[\mathrm{M}+\mathrm{H}^{+}\right]$370.0516, found 370.0511<smiles>COc1c(OC)c([Se]c2ccccc2)c(CC(=O)NC(C)C)c([Se]c2ccccc2)c1OC</smiles>

$N$-isopropyl-2-(3,4,5-trimethoxy-2,6-bis(phenylselanyl)phenyl)acetamide(3ra):

The general procedure A was followed using $N$-isopropyl-2-(3,4,5-trimethoxyphenyl) acetamide (1r) (66.8mg, $0.25 \mathrm{mmol})$ and 1,2-diphenyldiselane (2a) (156.0 mg, 0.5 mmol). Purification by column chromatography (Petroleum/DCE: $2 / 1 \rightarrow$ Petroleum/EtOAc: $4 / 1, \mathrm{Rf}=0.45)$ yielded 3ra $(80 \mathrm{mg}, 55 \%)$ as a yellow oil. M.p = $112-114{ }^{\circ} \mathrm{C} .{ }^{1} \mathrm{H}$ NMR $(600 \mathrm{MHz}) \delta=7.22(\mathrm{~m}, 4 \mathrm{H}), 7.19-7.16(\mathrm{~m}, 4 \mathrm{H}), 7.15-7.11$ 
(m, 2H), $5.16(\mathrm{~s}, 1 \mathrm{H}), 4.28(\mathrm{~s}, 2 \mathrm{H}), 3.94-3.86(\mathrm{~m}, 1 \mathrm{H}), 3.85(\mathrm{~s}, 3 \mathrm{H}), 3.71(\mathrm{~s}, 6 \mathrm{H}), 0.93$ $(\mathrm{d}, J=6.4 \mathrm{~Hz}, 6 \mathrm{H}) .{ }^{13} \mathrm{C} \mathrm{NMR}(150 \mathrm{MHz}) \delta=169.2,157.0,146.0,140.4,133.5,129.6$, 129.2, 126.26, 121.9, 61.2, 60.9, 45.8, 41.6, 22.5. HR-MS(ESI) $\mathrm{m} / z$ calcd for: $\mathrm{C}_{26} \mathrm{H}_{30} \mathrm{NO}_{4}{ }^{80} \mathrm{Se}_{2}{ }^{+}\left[\mathrm{M}+\mathrm{H}^{+}\right] 580.0050$, found 580.0048 .<smiles>Cc1ccc([Se]c2ccc(F)cc2)c(CC(=O)NC(C)C)c1</smiles>

2-(2-((4-bromophenyl)selanyl)-5-methylphenyl)- $N$-isopropylacetamide (3ib): The general procedure A was followed using $N$-isopropyl-2-(m-tolyl)acetamide (1i) (48 mg, $0.25 \mathrm{mmol}$ ) and 1,2-bis(4-fluorophenyl)diselane (2b) (175.0 $\mathrm{mg}, 0.5 \mathrm{mmol}$ ). Purification by column chromatography (Petroleum/DCE: 2/1 $\rightarrow$ Petroleum/EtOAc: 4/1, $\mathrm{Rf}=0.48)$ yielded 3ib $(69.4 \mathrm{mg}, 76 \%)$ as a white solid. M.p $=136-137{ }^{\circ} \mathrm{C} .{ }^{1} \mathrm{H}$ NMR $(600 \mathrm{MHz}) \delta=7.35(\mathrm{~d}, J=7.6 \mathrm{~Hz}, 1 \mathrm{H}), 7.31-7.30(\mathrm{~m}, 2 \mathrm{H}), 7.19(\mathrm{~s}, 1 \mathrm{H}), 6.98(\mathrm{~d}, J=$ $7.5 \mathrm{~Hz}, 1 \mathrm{H}), 6.93(\mathrm{~m}, 2 \mathrm{H}), 5.30(\mathrm{~s}, 1 \mathrm{H}), 4.09-3.92(\mathrm{~m}, 1 \mathrm{H}), 3.65(\mathrm{~s}, 2 \mathrm{H}), 2.32(\mathrm{~s}, 3 \mathrm{H})$, $1.01(\mathrm{~d}, J=6.6 \mathrm{~Hz}, 6 \mathrm{H}) .{ }^{13} \mathrm{C} \mathrm{NMR}(150 \mathrm{MHz}) \delta=169.5,162.3(\mathrm{~d}, J=263.9 \mathrm{~Hz}), 139.2$, $137.8,135.9,133.8$ (d, $J=7.9 \mathrm{~Hz}), 131.9,129.4,128.3,126.3,116.7$ (d, $J=21.7 \mathrm{~Hz})$, 44.2, 41.6, 22.6, 21.2. ${ }^{19} \mathrm{~F}$ NMR (565 MHz) $\delta=-114.4--114.7(\mathrm{~m})$. HR-MS(ESI) $\mathrm{m} / \mathrm{z}$ calcd for: $\mathrm{C}_{18} \mathrm{H}_{20} \mathrm{FNONa}^{80} \mathrm{Se}^{+}\left[\mathrm{M}+\mathrm{Na}^{+}\right]$388.0586, found 388.0585.<smiles>COc1cc(CC(=O)NC(C)C)c([Se]c2ccc([18F])cc2)cc1OC</smiles>

2-(2-((4-fluorophenyl)selanyl)-4,5-dimethoxyphenyl)- $N$-isopropylacetamide (3lb): The general procedure A was followed using 2-(3,4-dimethoxyphenyl)- $N$ isopropylacetamide (11) (59.3 mg, $0.25 \mathrm{mmol}$ ) and 1,2-bis(4-fluorophenyl)diselane (2b) 
(175.0 mg, $0.5 \mathrm{mmol}$ ). Purification by column chromatography (Petroleum/DCE: 2/1 $\rightarrow$ Petroleum/EtOAc: $4 / 1, \mathrm{Rf}=0.29)$ yielded $\mathbf{3 l b}(77 \mathrm{mg}, 75 \%)$ as a white solid. M.p $=145-146{ }^{\circ} \mathrm{C} .{ }^{1} \mathrm{H}$ NMR $(600 \mathrm{MHz}) \delta=7.23-7.20(\mathrm{~m}, 2 \mathrm{H}), 7.07$ (s, 1H), $6.96-6.91$ (m, 3H), $5.18(\mathrm{~s}, 1 \mathrm{H}), 3.95-3.92(\mathrm{~m}, 1 \mathrm{H}), 3.89(\mathrm{~s}, 3 \mathrm{H}), 3.80(\mathrm{~s}, 3 \mathrm{H}), 3.65(\mathrm{~s}, 2 \mathrm{H}), 0.98$ $(\mathrm{d}, J=6.4 \mathrm{~Hz}, 6 \mathrm{H}) .{ }^{13} \mathrm{C} \mathrm{NMR}(150 \mathrm{MHz}) \delta=169.8,162.1(\mathrm{~d}, J=241.3 \mathrm{~Hz}), 150.1$, 148.5, 132.4 (d, $J=7.4 \mathrm{~Hz}), 131.8,127.1,120.8,119.5,116.7$ (d, $J=21.5 \mathrm{~Hz}), 113.4$, 56.2, 56.1, 44.0, 41.6, 22.6. ${ }^{19} \mathrm{~F}$ NMR (565 MHz) $\delta=-115.0--115.3(\mathrm{~m})$. HR-MS(ESI) $m / z$ calcd for: $\mathrm{C}_{19} \mathrm{H}_{23} \mathrm{FNO}_{3}{ }^{80} \mathrm{Se}^{+}\left[\mathrm{M}+\mathrm{H}^{+}\right] 412.0822$, found 412.0819 .<smiles>CCCNC(=O)Cc1cc2c(cc1Oc1ccc([18OH])cc1)OCO2</smiles>

\section{2-(6-((4-fluorophenyl)selanyl)benzo $[d][1,3]$ dioxol-5-yl)- $N$-isopropylacetamide}

(3mb): The general procedure $\mathbf{A}$ was followed using 2-(benzo[d] [1,3]dioxol-5-yl) $-N$ isopropylacetamide (1m) $(55.3 \mathrm{mg}, 0.25 \mathrm{mmol}$ ) and 1,2-bis(4-fluorophenyl)diselane (2b) (175.0 mg, $0.5 \mathrm{mmol})$. Purification by column chromatography (Petroleum/DCE: $2 / 1 \rightarrow$ Petroleum/EtOAc: $4 / 1, \mathrm{Rf}=0.31)$ yielded $\mathbf{3 m b}(80 \mathrm{mg}, 81 \%)$ as a white solid. M.p $=163-164{ }^{\circ} \mathrm{C} .{ }^{1} \mathrm{H}$ NMR $(600 \mathrm{MHz}) \delta=7.30-7.26(\mathrm{~m}, 2 \mathrm{H}), 6.98-6.92(\mathrm{~m}, 3 \mathrm{H})$, $6.88(\mathrm{~s}, 1 \mathrm{H}), 5.96(\mathrm{~s}, 2 \mathrm{H}), 5.22(\mathrm{~s}, 1 \mathrm{H}), 4.05-3.89(\mathrm{~m}, 1 \mathrm{H}), 3.63(\mathrm{~s}, 2 \mathrm{H}), 1.01(\mathrm{~d}, J=$ $6.4 \mathrm{~Hz}, 6 \mathrm{H}) .{ }^{13} \mathrm{C}$ NMR $(150 \mathrm{MHz}) \delta=169.5,162.3(\mathrm{~d}, J=252.1 \mathrm{~Hz}), 148.8,147.6$, $133.5(\mathrm{~d}, J=7.6 \mathrm{~Hz}), 132.1,126.5,122.7,116.7$ (d, $J=21.6 \mathrm{~Hz}), 115.8,110.9,101.7$, 44.1, 41.6, 22.7. ${ }^{19} \mathrm{~F}$ NMR (565 MHz) $\delta=-117.4--114.7(\mathrm{~m})$. HR-MS(ESI) $\mathrm{m} / z$ calcd for: $\mathrm{C}_{18} \mathrm{H}_{19} \mathrm{FNO}_{3}{ }^{80} \mathrm{Se}^{+}\left[\mathrm{M}+\mathrm{H}^{+}\right]$396.0509, found 396.0505.<smiles>Cc1ccc([Se]c2ccc(Cl)cc2)c(CC(=O)NC(C)C)c1</smiles> 
2-(2-((4-chlorophenyl)selanyl)-5-methylphenyl)- $N$-isopropylacetamide (3ic): The general procedure A was followed using $N$-isopropyl-2-( $m$-tolyl)acetamide (1i) (48 mg, $0.25 \mathrm{mmol}$ ) and 1,2-bis(4-chlorophenyl)diselane (2c) (191.0 $\mathrm{mg}, 0.5 \mathrm{mmol})$. Purification by column chromatography (Petroleum/DCE: $2 / 1 \rightarrow$ Petroleum/EtOAc: 4/1, $\mathrm{Rf}=0.46)$ yielded 3ic $(55 \mathrm{mg}, 58 \%)$ as a white solid. M.p $=121-123{ }^{\circ} \mathrm{C} .{ }^{1} \mathrm{H}$ NMR $(600$ MHz) $\delta=7.42(\mathrm{~d}, J=7.8 \mathrm{~Hz}, 1 \mathrm{H}), 7.22(\mathrm{~s}, 1 \mathrm{H}), 7.20-7.15(\mathrm{~m}, 4 \mathrm{H}), 7.02(\mathrm{~d}, J=7.9$ Hz, 1H), 5.37 (s, 1H), $4.06-3.87(\mathrm{~m}, 1 \mathrm{H}), 3.64(\mathrm{~s}, 2 \mathrm{H}), 2.34$ (s, 3H), 1.00 (d, J = 6.7 $\mathrm{Hz}, 6 \mathrm{H}) .{ }^{13} \mathrm{C}$ NMR $(150 \mathrm{MHz}) \delta=169.4,139.8,138.4,136.8,133.0,132.4,132.0$, 130.5, 129.6, 129.5, 127.2, 44.3, 41.6, 22.6, 21.2. HR-MS(ESI) $\mathrm{m} / z$ calcd for: $\mathrm{C}_{18} \mathrm{H}_{21} \mathrm{ClNO}^{80} \mathrm{Se}^{+}\left[\mathrm{M}+\mathrm{H}^{+}\right] 404.0291$, found 404.0287<smiles>Cc1ccc([Se]c2ccc(C(F)(F)F)cc2)c(CC(=O)NC(C)C)c1</smiles>

$N$-isopropyl-2-(5-methyl-2-((4-(trifluoromethyl)phenyl)selanyl)phenyl) acetamide (3id): The general procedure A was followed using $N$-isopropyl-2-( $m$-tolyl)acetamide (1i) (48 mg, $0.25 \mathrm{mmol}$ ) and 1,2-bis(4-(trifluoromethyl)phenyl)diselane (2d) (225.0 mg, $0.5 \mathrm{mmol}$ ). Purification by column chromatography (Petroleum/DCE: 2/1 $\rightarrow$ Petroleum/EtOAc: $4 / 1, \mathrm{Rf}=0.47)$ yielded 3id $(83 \mathrm{mg}, 80 \%)$ as a white solid. M.p = $131-133{ }^{\circ} \mathrm{C} .{ }^{1} \mathrm{H}$ NMR $(600 \mathrm{MHz}) \delta=7.52(\mathrm{~d}, J=7.8 \mathrm{~Hz}, 1 \mathrm{H}), 7.40(\mathrm{~d}, J=8.3 \mathrm{~Hz}, 2 \mathrm{H})$, $7.28(\mathrm{~s}, 1 \mathrm{H}), 7.24(\mathrm{~d}, J=8.3 \mathrm{~Hz}, 2 \mathrm{H}), 7.07(\mathrm{~d}, J=8.0 \mathrm{~Hz}, 1 \mathrm{H}), 5.24(\mathrm{~s}, 1 \mathrm{H}), 3.99-3.94$ $(\mathrm{m}, 1 \mathrm{H}), 3.64(\mathrm{~s}, 2 \mathrm{H}), 2.37(\mathrm{~s}, 3 \mathrm{H}), 0.97(\mathrm{~d}, J=6.5 \mathrm{~Hz}, 6 \mathrm{H}) \cdot{ }^{13} \mathrm{C} \mathrm{NMR}(150 \mathrm{MHz}) \delta=$ $169.3,140.6,139.3,138.5,138.0,132.2,129.7$ (q, $J=11.0 \mathrm{~Hz}), 129.6,128.5$ (q, $J=$ $33.4 \mathrm{~Hz}), 126.8,126.0$ (q, $J=3.8 \mathrm{~Hz}), 124.1(\mathrm{q}, J=272.4 \mathrm{~Hz}), 44.3,41.6,22.6,21.3$. ${ }^{19} \mathrm{~F}$ NMR (565 MHz) $\delta=-62.49$ (s). HR-MS(ESI) $m / z$ calcd for: $\mathrm{C}_{19} \mathrm{H}_{20} \mathrm{~F}_{3} \mathrm{NONa}^{80} \mathrm{Se}^{+}$ $\left[\mathrm{M}+\mathrm{Na}^{+}\right]$438.0554, found 438.0551. 
<smiles>Cc1ccc([Se]c2ccc(C)cc2CC(=O)NC(C)C)c(C)c1</smiles>

$\mathrm{N}$-isopropyl-2-(5-methyl-2-(p-tolylselanyl)phenyl)acetamide (3ie): The general procedure A was followed using $N$-isopropyl-2-( $m$-tolyl)acetamide (1i) (48 $\mathrm{mg}, 0.25$ $\mathrm{mmol})$ and 1,2-di-p-tolyldiselane (2e) $(176.0 \mathrm{mg}, 0.5 \mathrm{mmol})$. Purification by column chromatography (Petroleum/DCE: $2 / 1 \rightarrow$ Petroleum/EtOAc: $4 / 1, \mathrm{Rf}=0.45$ ) yielded 3ie $(41 \mathrm{mg}, 46 \%)$ as a white solid. M.p $=105-106{ }^{\circ} \mathrm{C} .{ }^{1} \mathrm{H} \mathrm{NMR}(600 \mathrm{MHz}) \delta=7.37(\mathrm{~d}, J$ $=7.8 \mathrm{~Hz}, 1 \mathrm{H}), 7.21(\mathrm{~d}, J=8.0 \mathrm{~Hz}, 2 \mathrm{H}), 7.20(\mathrm{~d}, J=1.2 \mathrm{~Hz}, 1 \mathrm{H}), 7.04(\mathrm{~d}, J=8.0 \mathrm{~Hz}$, $2 \mathrm{H}), 6.98(\mathrm{dd}, J=8.0,1.2 \mathrm{~Hz}, 1 \mathrm{H}), 5.41-5.07(\mathrm{~s}, 1 \mathrm{H}), 4.06-3.92(\mathrm{~m}, 1 \mathrm{H}), 3.66(\mathrm{~s}$, 2H), $2.31(\mathrm{~s}, 3 \mathrm{H}), 2.29(\mathrm{~s}, 3 \mathrm{H}), 0.99(\mathrm{~d}, J=6.7 \mathrm{~Hz}, 6 \mathrm{H}) .{ }^{13} \mathrm{C} \mathrm{NMR}(151 \mathrm{MHz}) \delta=169.7$, 138.9, 137.8, 137.2, 135.9, 132.1, 131.7, 130.4, 129.3, 128.4, 127.9, 44.229, 41.549, 22.7 22.6, 21.2. HR-MS(ESI) $m / z$ calcd for: $\mathrm{C}_{19} \mathrm{H}_{23} \mathrm{NONa}^{80} \mathrm{Se}^{+}\left[\mathrm{M}+\mathrm{Na}^{+}\right]$384.0837, found 384.0839<smiles>Cc1ccc([Se]c2cccc(Cl)c2)c(CC(=O)NC(C)C)c1</smiles>

2-(2-((3-chlorophenyl)selanyl)-5-methylphenyl)- $N$-isopropylacetamide (3if): The general procedure A was followed using $N$-isopropyl-2-( $m$-tolyl)acetamide (1i) (48 mg, $0.25 \mathrm{mmol}$ ) and 1,2-bis(3-chlorophenyl)diselane (2f) $(191.0 \mathrm{mg}, 0.5 \mathrm{mmol})$. Purification by column chromatography (Petroleum/DCE: $2 / 1 \rightarrow$ Petroleum/EtOAc: $4 / 1, \mathrm{Rf}=0.47)$ yielded 3if $(57 \mathrm{mg}, 60 \%)$ as a white solid. M.p $=91-92{ }^{\circ} \mathrm{C} .{ }^{1} \mathrm{H}$ NMR $(600 \mathrm{MHz}) \delta=7.48(\mathrm{~d}, J=7.8 \mathrm{~Hz}, 1 \mathrm{H}), 7.25(\mathrm{~s}, 1 \mathrm{H}), 7.19(\mathrm{~s}, 1 \mathrm{H}), 7.16-7.12(\mathrm{~m}, 1 \mathrm{H})$, $7.12-7.08(\mathrm{~m}, 2 \mathrm{H}), 7.06-7.03(\mathrm{~d}, J=8.0 \mathrm{~Hz}, 1 \mathrm{H}), 5.52-5.12(\mathrm{~s}, 1 \mathrm{H}), 4.03-3.91$ $(\mathrm{m}, 1 \mathrm{H}), 3.65(\mathrm{~s}, 2 \mathrm{H}), 2.35(\mathrm{~s}, 3 \mathrm{H}), 0.99(\mathrm{~d}, J=6.7 \mathrm{~Hz}, 6 \mathrm{H}) \cdot{ }^{13} \mathrm{C} \mathrm{NMR}(150 \mathrm{MHz}) \delta=$ 
169.5, 140.1, 138.8, 137.4, 135.2, 134.4, 1321, 130.4, 130.0, 129.6, 128.6, 126.9, 126.4, 44.4, 41.6, 22.6, 21.3. HR-MS(ESI) $m / z$ calcd for: $\mathrm{C}_{18} \mathrm{H}_{21} \mathrm{ClNO}^{80} \mathrm{Se}^{+}\left[\mathrm{M}+\mathrm{H}^{+}\right]$382.0471, found 382.0468 .<smiles>Cc1ccc(OC(=O)Cc2cccc(C(F)(F)F)c2)c(C(=O)NC(C)C)c1</smiles>

$N$-isopropyl-2-(5-methyl-2-((3-(trifluoromethyl)phenyl)selanyl)phenyl)acetamide

(3ig): The general procedure A was followed using $N$-isopropyl-2-( $m$-tolyl)acetamide (1i) (48 mg, $0.25 \mathrm{mmol})$ and 1,2-bis(3-(trifluoromethyl)phenyl)diselane (2g) (225.0 $\mathrm{mg}, 0.5 \mathrm{mmol}$ ). Purification by column chromatography (Petroleum/DCE: $2 / 1 \rightarrow$ Petroleum/EtOAc: $4 / 1, \mathrm{Rf}=0.46)$ yielded 3ig $(79 \mathrm{mg}, 76 \%)$ as a white solid. M.p = 131-133 ${ }^{\circ} \mathrm{C} .{ }^{1} \mathrm{H}$ NMR $(600 \mathrm{MHz}) \delta=7.51(\mathrm{~s}, 1 \mathrm{H}), 7.47(\mathrm{~d}, J=7.8 \mathrm{~Hz}, 1 \mathrm{H}), 7.42(\mathrm{~d}, J$ $=7.5 \mathrm{~Hz}, 1 \mathrm{H}), 7.33(\mathrm{~d}, J=7.7 \mathrm{~Hz}, 1 \mathrm{H}), 7.29-7.27(\mathrm{~m}, 1 \mathrm{H}), 7.26(\mathrm{~s}, 1 \mathrm{H}), 7.05(\mathrm{~d}, J=$ $7.6 \mathrm{~Hz}, 1 \mathrm{H}), 5.24(\mathrm{~s}, 1 \mathrm{H}), 4.00-3.91(\mathrm{~m}, 1 \mathrm{H}), 3.65(\mathrm{~s}, 2 \mathrm{H}), 2.36(\mathrm{~s}, 3 \mathrm{H}), 0.99$ (d, $J=$ $5.6 \mathrm{~Hz}, 6 \mathrm{H}) .{ }^{13} \mathrm{C}$ NMR $(150 \mathrm{MHz}) \delta=169.4,140.2,138.8,137.3,133.9,133.6,132.2$, $131.6(\mathrm{q}, J=31.6 \mathrm{~Hz}), 129.7(\mathrm{q}, J=5.7 \mathrm{~Hz}), 127.6,126.9(\mathrm{q}, J=3.6 \mathrm{~Hz}), 126.2,123.7$ $(\mathrm{q}, J=272.7 \mathrm{~Hz}), 123.4(\mathrm{q}, J=3.2 \mathrm{~Hz}), 44.3,41.6,22.6,21.3 .{ }^{19} \mathrm{~F}$ NMR $(565 \mathrm{MHz}) \delta$ $=-62.70(\mathrm{~s}) . \mathrm{HR}-\mathrm{MS}(\mathrm{ESI}) \mathrm{m} / \mathrm{z}$ calcd for: $\mathrm{C}_{19} \mathrm{H}_{21} \mathrm{~F}_{3} \mathrm{NO}^{80} \mathrm{Se}^{+}\left[\mathrm{M}+\mathrm{H}^{+}\right] 416.0735$, found 416.0726<smiles>Cc1ccc([Se]c2ccc([AsH2])cc2F)c(CC(=O)NC(C)C)c1</smiles>

2-(2-((2-fluorophenyl)selanyl)-5-methylphenyl)- $N$-isopropylacetamide (3ih): The general procedure A was followed using $N$-isopropyl-2-( $m$-tolyl)acetamide (1i) (48 mg, $0.25 \mathrm{mmol}$ ) and 1,2-bis(2-fluorophenyl)diselane (2h) (175.0mg, $0.5 \mathrm{mmol}$ ). 
Purification by column chromatography (Petroleum/DCE: $2 / 1 \rightarrow$ Petroleum/EtOAc: $4 / 1$, $\mathrm{Rf}=0.43)$ yielded $3 \mathrm{ih}(59 \mathrm{mg}, 65 \%)$ as a white solid. M.p $=112-114{ }^{\circ} \mathrm{C} .{ }^{1} \mathrm{H}$ NMR $(600$ MHz) $\delta=7.45(\mathrm{~d}, J=7.8 \mathrm{~Hz}, 1 \mathrm{H}), 7.25(\mathrm{~d}, J=1.0 \mathrm{~Hz}, 1 \mathrm{H}), 7.21-7.15(\mathrm{~m}, 1 \mathrm{H}), 7.05$ - $7.00(\mathrm{~m}, 2 \mathrm{H}), 6.95-6.94(\mathrm{~m}, 2 \mathrm{H}), 5.37(\mathrm{~s}, 1 \mathrm{H}), 4.01-3.88(\mathrm{~m}, 1 \mathrm{H}), 3.66(\mathrm{~s}, 2 \mathrm{H})$, $2.34(\mathrm{~s}, 3 \mathrm{H}), 1.00(\mathrm{~d}, J=6.4 \mathrm{~Hz}, 6 \mathrm{H}) .{ }^{13} \mathrm{C} \mathrm{NMR}(151 \mathrm{MHz}) \delta=169.5,161.3(\mathrm{~d}, J=$ $243.2 \mathrm{~Hz}$ ), 139.9, 138.9, 137.3, 132.3, 132.0, 129.5, 128.7 (d, J= 7.6 Hz), 125.2, 125.1 (d, $J=3.2 \mathrm{~Hz}), 119.3(\mathrm{~d}, J=20.5 \mathrm{~Hz}), 115.6(\mathrm{~d}, J=22.6 \mathrm{~Hz}), 44.2,41.6,22.5,21.3$. ${ }^{19} \mathrm{~F}$ NMR (565 MHz) $\delta=-104.6--104.8$ (m). HR-MS(ESI) $\mathrm{m} / z$ calcd for: $\mathrm{C}_{18} \mathrm{H}_{21} \mathrm{FNO}^{80} \mathrm{Se}^{+}\left[\mathrm{M}+\mathrm{Na}^{+}\right]$366.0767, found 366.0759.<smiles>Cc1ccc([Se]c2ccccc2Br)c(CC(=O)NC(C)C)c1</smiles>

3ii

2-(2-((2-bromophenyl)selanyl)-5-methylphenyl)- $N$-isopropylacetamide (3ii): The general procedure A was followed using $N$-isopropyl-2-( $m$-tolyl)acetamide (1i) (48 mg, $0.25 \mathrm{mmol}$ ) and 1,2-bis(2-bromophenyl)diselane (2i) $(235.0 \mathrm{mg}, 0.5 \mathrm{mmol})$. Purification by column chromatography (Petroleum/DCE: 2/1 $\rightarrow$ Petroleum/EtOAc: 4/1, $\mathrm{Rf}=0.42)$ yielded 3ii $(77 \mathrm{mg}, 72 \%)$ as a white solid. M.p $=112-114{ }^{\circ} \mathrm{C} .{ }^{1} \mathrm{H}$ NMR $(600$ MHz) $\delta=7.56(\mathrm{~d}, J=7.9 \mathrm{~Hz}, 1 \mathrm{H}), 7.47(\mathrm{dd}, J=7.8,1.3 \mathrm{~Hz}, 1 \mathrm{H}), 7.35$ (s, 1H), 7.09 $(\mathrm{dd}, J=6.7,1.0 \mathrm{~Hz}, 1 \mathrm{H}), 7.01-7.00(\mathrm{~m}, 2 \mathrm{H}), 6.62(\mathrm{dd}, J=7.7,1.7 \mathrm{~Hz}, 1 \mathrm{H}), 5.39$ (s, $1 \mathrm{H}), 3.96-3.85(\mathrm{~m}, 1 \mathrm{H}), 3.62(\mathrm{~s}, 2 \mathrm{H}), 2.38(\mathrm{~s}, 3 \mathrm{H}), 0.97(\mathrm{~d}, J=6.4 \mathrm{~Hz}, 6 \mathrm{H}) .{ }^{13} \mathrm{C} \mathrm{NMR}$ (150 MHz) $\delta=169.4,140.9,140.1,138.7,136.5,132.8,132.4,129.7,129.6,128.1$, 127.3, 125.7, 122.9, 44.3, 41.6, 22.5, 21.4. HR-MS(ESI) $\mathrm{m} / \mathrm{z}$ calcd for: $\mathrm{C}_{18} \mathrm{H}_{21} \mathrm{BrNO}^{80} \mathrm{Se}^{+}\left[\mathrm{M}+\mathrm{H}^{+}\right]$425.9966, found 425.9963 .<smiles>CCCOC(=O)Cc1ccc(OC)cc1[Se]c1ccccc1</smiles>

$3 s a$ 
Isopropyl 2-(4-methoxy-2-(phenylselanyl)phenyl)acetate (3sa): The general procedure B was followed using isopropyl 2-(4-methoxyphenyl)acetate (1s) (52 mg, $0.25 \mathrm{mmol}$ ) and 1,2-diphenyldiselane (2a) $(156.0 \mathrm{mg}, 0.5 \mathrm{mmol})$. Purification by column chromatography (Petroleum/EtOAc: $10 / 1, \mathrm{Rf}=0.50)$ yielded 3sa $(66 \mathrm{mg}, 73 \%)$ as a colorless oil. ${ }^{1} \mathrm{H}$ NMR $(600 \mathrm{MHz}) \delta=7.56-7.54(\mathrm{~m}, 2 \mathrm{H}), 7.35-7.29(\mathrm{~m}, 3 \mathrm{H})$, $7.10(\mathrm{dd}, J=8.3,2.1 \mathrm{~Hz}, 1 \mathrm{H}), 6.88(\mathrm{~d}, J=2.1 \mathrm{~Hz}, 1 \mathrm{H}), 6.80(\mathrm{~d}, J=8.3 \mathrm{~Hz}, 1 \mathrm{H}), 4.92$ (hept, $J=6.3 \mathrm{~Hz}, 1 \mathrm{H}), 3.86(\mathrm{~s}, 3 \mathrm{H}), 3.36(\mathrm{~s}, 2 \mathrm{H}), 1.14(\mathrm{~d}, J=6.3 \mathrm{~Hz}, 6 \mathrm{H}) .{ }^{13} \mathrm{C} \mathrm{NMR}$ (150 MHz) $\delta=171.2,155.9,135.2,131.9,129.5,128.7,128.1,127.6,126.6,121.7$, 110.5, 68.2, 56.1, 40.8, 21.8. HR-MS(ESI) $\mathrm{m} / z$ calcd for: $\mathrm{C}_{18} \mathrm{H}_{21} \mathrm{NO}_{3}{ }^{80} \mathrm{Se}^{+}\left[\mathrm{M}+\mathrm{H}^{+}\right]$ 365.0650 , found 365.0649 .<smiles>CCCOC(=O)Cc1ccc(OC)cc1[Se]c1ccc(C(C)(C)C)cc1</smiles>

Isopropyl 2-(4-methoxy-2-(p-tolylselanyl)phenyl)acetate (3se): The general procedure B was followed using isopropyl 2-(4-methoxyphenyl)acetate (1s) (52 mg, $0.25 \mathrm{mmol}$ ) and 1,2-di-p-tolyldiselane (2e) $(171.0 \mathrm{mg}, 0.5 \mathrm{mmol})$ and. Purification by column chromatography (Petroleum/EtOAc: 10/1, Rf=0.55) yielded 3se (70 mg, $74 \%$ ) as a colorless oil. ${ }^{1} \mathrm{H}$ NMR $(600 \mathrm{MHz}) \delta=7.42(\mathrm{~d}, J=8.3 \mathrm{~Hz}, 2 \mathrm{H}), 7.38(\mathrm{~d}, J=8.3 \mathrm{~Hz}$, $2 \mathrm{H}), 7.13(\mathrm{dd}, J=8.3,2.0 \mathrm{~Hz}, 1 \mathrm{H}), 6.94(\mathrm{~d}, J=2.0 \mathrm{~Hz}, 1 \mathrm{H}), 6.81(\mathrm{~d}, J=8.3 \mathrm{~Hz}, 1 \mathrm{H})$, 4.97 (hept, $J=6.4 \mathrm{~Hz}, 1 \mathrm{H}), 3.84(\mathrm{~s}, 3 \mathrm{H}), 3.40$ (s, 2H), 2.32 (s, 3H), 1.16 (d, $J=6.4 \mathrm{~Hz}$, $6 \mathrm{H}) .{ }^{13} \mathrm{C}$ NMR $(150 \mathrm{MHz}) \delta=171.2,155.5,138.4,135.9,130.8,130.4,128.2,127.5$, 124.0, 122.6, 110.3, 68.1, 56.0, 40.8, 21.7, 21.3. HR-MS(ESI) $\mathrm{m} / \mathrm{z}$ calcd for: $\mathrm{C}_{19} \mathrm{H}_{23} \mathrm{NO}_{3}{ }^{80} \mathrm{Se}^{+}\left[\mathrm{M}+\mathrm{H}^{+}\right] 379.0807$, found 379.0805 . 
<smiles>CCCOC(=O)Cc1ccc(OC)cc1[Se]c1ccc(Br)cc1</smiles>

Isopropyl 2-(2-((4-bromophenyl)selanyl)-4-methoxyphenyl)acetate (3sj): The general procedure $\mathbf{B}$ was followed using isopropyl 2-(4-methoxyphenyl)acetate (1s) (52 $\mathrm{mg}, 0.25 \mathrm{mmol}$ ) and 1,2-bis(4-bromophenyl)diselane (2j) (235.0 mg, $0.5 \mathrm{mmol})$ and. Purification by column chromatography (Petroleum/EtOAc: $10 / 1, \mathrm{Rf}=0.53$ ) yielded 3sj $(87 \mathrm{mg}, 80 \%)$ as a colorless oil. ${ }^{1} \mathrm{H}$ NMR $(600 \mathrm{MHz}) \delta=7.42(\mathrm{~d}, J=8.3 \mathrm{~Hz}, 2 \mathrm{H})$, $7.38(\mathrm{~d}, J=8.3 \mathrm{~Hz}, 2 \mathrm{H}), 7.13(\mathrm{dd}, J=8.3,2.0 \mathrm{~Hz}, 1 \mathrm{H}), 6.94(\mathrm{~d}, J=2.0 \mathrm{~Hz}, 1 \mathrm{H}), 6.81$ (d, $J=8.3 \mathrm{~Hz}, 1 \mathrm{H}), 4.97$ (hept, $J=6.4 \mathrm{~Hz}, 1 \mathrm{H}), 3.84$ (s, 3H), 3.40 (s, 2H), 1.16 (d, $J=$ $6.4 \mathrm{~Hz}, 6 \mathrm{H}) .{ }^{13} \mathrm{C}$ NMR $(150 \mathrm{MHz}) \delta=171.2,156.2,136.2,132.6,132.5,129.3,127.9$, $127.7,122.4,120.8,110.7,68.3,56.1,40.7,21.8$. HR-MS(ESI) $\mathrm{m} / z$ calcd for: $\mathrm{C}_{18} \mathrm{H}_{20} \mathrm{BrNO}_{3}{ }^{80} \mathrm{Se}^{+}\left[\mathrm{M}+\mathrm{H}^{+}\right] 442.9755$, found 442.9756<smiles>CCCOC(=O)Cc1ccc(OC)cc1[Se]c1ccccc1F</smiles>

Isopropyl 2-(2-((2-fluorophenyl)selanyl)-4-methoxyphenyl)acetate (3sh): The general procedure B was followed using isopropyl 2-(4-methoxyphenyl)acetate (1s) (52 $\mathrm{mg}, 0.25 \mathrm{mmol}$ ) and 1,2-bis(2-fluorophenyl)diselane (2h) (175.0mg, $0.5 \mathrm{mmol}$ ). Purification by column chromatography (Petroleum/EtOAc: $10 / 1, \mathrm{Rf}=0.53$ ) yielded 3sh $(73 \mathrm{mg}, 77 \%)$ as a colorless oil. ${ }^{1} \mathrm{H}$ NMR $(600 \mathrm{MHz}) \delta=7.40-7.36(\mathrm{~m}, 1 \mathrm{H}), 7.34$ $-7.29(\mathrm{~m}, 1 \mathrm{H}), 7.14(\mathrm{dd}, J=8.3,1.8 \mathrm{~Hz}, 1 \mathrm{H}), 7.11-7.10(\mathrm{~m}, 1 \mathrm{H}), 7.06-7.04(\mathrm{~m}, 1 \mathrm{H})$, $6.94(\mathrm{~d}, J=1.8 \mathrm{~Hz}, 1 \mathrm{H}), 6.82(\mathrm{~d}, J=8.3 \mathrm{~Hz}, 1 \mathrm{H}), 4.93$ (hept, $J=6.3 \mathrm{~Hz}, 1 \mathrm{H}), 3.85$ (s, $3 \mathrm{H}), 3.39(\mathrm{~s}, 2 \mathrm{H}), 1.15(\mathrm{~d}, J=6.3 \mathrm{~Hz}, 6 \mathrm{H}) .{ }^{13} \mathrm{C} \mathrm{NMR}(151 \mathrm{MHz}) \delta=171.1,162.7(\mathrm{~d}, J$ 
$=246.2 \mathrm{~Hz}), 156.5,136.2(\mathrm{~d}, J=2.5 \mathrm{~Hz}), 132.7,130.2(\mathrm{~d}, J=7.7 \mathrm{~Hz}), 129.4,127.7$, $125.1(\mathrm{~d}, J=3.2 \mathrm{~Hz}), 119.3,115.9(\mathrm{~d}, J=22.3 \mathrm{~Hz}), 115.8(\mathrm{~d}, J=23.7 \mathrm{~Hz}), 110.7,68.2$, 56.1, 40.8, 21.8. ${ }^{19} \mathrm{~F}$ NMR (565 MHz) $\delta=-102.34$ (s). HR-MS(ESI) $\mathrm{m} / \mathrm{z}$ calcd for: $\mathrm{C}_{18} \mathrm{H}_{18} \mathrm{FNO}_{3}{ }^{80} \mathrm{Se}^{+}\left[\mathrm{M}-\mathrm{H}^{+}\right] 381.0410$, found 382.0411<smiles>CCCOC(=O)Cc1ccc(OC)cc1[Se]c1ccccc1Br</smiles>

3si

Isopropyl 2-(2-((2-bromophenyl)selanyl)-4-methoxyphenyl)acetate (3si): The general procedure $\mathbf{B}$ was followed using isopropyl 2-(4-methoxyphenyl)acetate (1s) (52 $\mathrm{mg}, \quad 0.25 \mathrm{mmol}$ ) and 1,2-bis(2-borophenyl)diselane (2i) (235.0mg, $0.5 \mathrm{mmol}$ ). Purification by column chromatography (Petroleum/EtOAc: $10 / 1, \mathrm{Rf}=0.47$ ) yielded 3si $(70 \mathrm{mg}, 63 \%)$ as a colorless oil. ${ }^{1} \mathrm{H}$ NMR $(600 \mathrm{MHz}) \delta=7.40-7.36(\mathrm{~m}, 1 \mathrm{H}), 7.34$ $-7.29(\mathrm{~m}, 1 \mathrm{H}), 7.14(\mathrm{dd}, J=8.3,1.8 \mathrm{~Hz}, 1 \mathrm{H}), 7.11-7.10(\mathrm{~m}, 1 \mathrm{H}), 7.06-7.04(\mathrm{~m}, 1 \mathrm{H})$, $6.94(\mathrm{~d}, J=1.8 \mathrm{~Hz}, 1 \mathrm{H}), 6.82(\mathrm{~d}, J=8.3 \mathrm{~Hz}, 1 \mathrm{H}), 4.93$ (hept, $J=6.3 \mathrm{~Hz}, 1 \mathrm{H}), 3.85$ (s, $3 \mathrm{H}), 3.39(\mathrm{~s}, 2 \mathrm{H}), 1.15(\mathrm{~d}, J=6.3 \mathrm{~Hz}, 6 \mathrm{H}) .{ }^{13} \mathrm{C} \mathrm{NMR}(150 \mathrm{MHz}) \delta=171.1,157.9,136.8$, 134.4, 132.8, 131.9, 131.2, 127.8, 127.8, 127.7, 124.9, 118.0, 111.1, 68.3, 56.1, 40.6, 21.8. HR-MS(ESI) $\mathrm{m} / z$ calcd for: $\mathrm{C}_{18} \mathrm{H}_{20} \mathrm{BrNO}_{3}{ }^{80} \mathrm{Se}^{+}\left[\mathrm{M}+\mathrm{H}^{+}\right]$442.9756, found 442.9758<smiles>CCCOC(=O)Cc1c([Se]c2ccccc2)csc1[Se]c1ccccc1</smiles>

3ta

Isopropyl 2-(2,4-bis(phenylselanyl)thiophen-3-yl)acetate (3ta): The general procedure B was followed using isopropyl 2-(thiophen-3-yl)acetate (1t) (46 mg, 0.25 mmol) and 1,2-diphenyldiselane (2a) (156.0mg, $0.5 \mathrm{mmol})$. Purification by column chromatography (Petroleum/EtOAc: $10 / 1, \mathrm{Rf}=0.69)$ yielded 3ta $(66 \mathrm{mg}, 53 \%)$ as a colorless oil. ${ }^{1} \mathrm{H}$ NMR $(600 \mathrm{MHz}) \delta=7.41-7.39(\mathrm{~m}, 2 \mathrm{H}), 7.31(\mathrm{~s}, 1 \mathrm{H}), 7.31-7.28$ 
(m, 2H), $7.27-7.23(\mathrm{~m}, 3 \mathrm{H}), 7.23-7.20(\mathrm{~m}, 3 \mathrm{H}), 4.94$ (hept, $J=6.3 \mathrm{~Hz}, 1 \mathrm{H}), 3.73(\mathrm{~s}$, $2 \mathrm{H}), 1.19(\mathrm{~d}, J=6.3 \mathrm{~Hz}, 6 \mathrm{H}) .{ }^{13} \mathrm{C} \mathrm{NMR}(151 \mathrm{MHz}) \delta=170.1,141.8,137.9,132.7$, $132.5,130.9,130.1,129.5,129.3,129.1,127.4,127.0,126.7,68.6,36.1,21.9$. HR$\mathrm{MS}(\mathrm{ESI}) \mathrm{m} / \mathrm{z}$ calcd for: $\mathrm{C}_{21} \mathrm{H}_{21} \mathrm{O}_{2} \mathrm{~S}^{80} \mathrm{Se}_{2}{ }^{+}\left[\mathrm{M}+\mathrm{H}^{+}\right]$496.9587, found 496.9585 .<smiles>CCCOC(=O)Cc1ccc(OC)cc1Sc1ccccc1</smiles>

Isopropyl 2-(4-methoxy-2-(phenylthio)phenyl)acetate (3sk): The general procedure B was followed using isopropyl 2-(4-methoxyphenyl)acetate (1s) (52 mg, $0.25 \mathrm{mmol})$ and 1,2-diphenyldisulfane (2k) (109.0 mg, $0.5 \mathrm{mmol})$. Purification by column chromatography (Petroleum/EtOAc: $10 / 1, \mathrm{Rf}=0.51)$ yielded 3sk $(63 \mathrm{mg}, 80 \%)$ as a colorless oil. ${ }^{1} \mathrm{H}$ NMR $(600 \mathrm{MHz}) \delta=7.34-7.31(\mathrm{~m}, 4 \mathrm{H}), 7.25-7.23(\mathrm{~m}, 1 \mathrm{H}), 7.15$ $(\mathrm{dd}, J=8.3,2.1 \mathrm{~Hz}, 1 \mathrm{H}), 7.01(\mathrm{~d}, J=2.1 \mathrm{~Hz}, 1 \mathrm{H}), 6.85(\mathrm{~d}, J=8.3 \mathrm{~Hz}, 1 \mathrm{H}), 4.92$ (hept, $J=6.2 \mathrm{~Hz}, 1 \mathrm{H}), 3.84(\mathrm{~s}, 3 \mathrm{H}), 3.41(\mathrm{~s}, 2 \mathrm{H}), 1.16(\mathrm{~d}, J=6.2 \mathrm{~Hz}, 6 \mathrm{H}) .{ }^{13} \mathrm{C} \mathrm{NMR}(150$ $\mathrm{MHz}) \delta=171.2$, 156.6, 136.7, 134.6, 132.6, 131.3, 129.3, 129.2, 127.1, 123.9, 111.0, 68.3, 56.1, 40.8, 21.8. HR-MS(ESI) $m / z$ calcd for: $\mathrm{C}_{18} \mathrm{H}_{21} \mathrm{O}_{3} \mathrm{~S}^{+}\left[\mathrm{M}+\mathrm{H}^{+}\right] 317.1206$, found 317.1205.<smiles>CCCOC(=O)Cc1ccc(OC)cc1Sc1ccc(C)cc1</smiles>

Isopropyl 2-(4-methoxy-2-(p-tolylthio)phenyl)acetate (3sl): The general procedure B was followed using isopropyl 2-(4-methoxyphenyl)acetate (1s) $(52 \mathrm{mg}, 0.25 \mathrm{mmol})$ and 1,2-di-p-tolyldisulfane (2l) $(123.0 \mathrm{mg}, 0.5 \mathrm{mmol})$. Purification by column chromatography (Petroleum/EtOAc: $10 / 1, \mathrm{Rf}=0.50)$ yielded 3sl $(59 \mathrm{mg}, 71 \%)$ as a colorless oil. ${ }^{1} \mathrm{H}$ NMR $(600 \mathrm{MHz}) \delta=7.29(\mathrm{~d}, J=8.1 \mathrm{~Hz}, 2 \mathrm{H}), 7.13(\mathrm{~d}, J=8.0 \mathrm{~Hz}, 2 \mathrm{H})$, 
$7.08(\mathrm{dd}, J=8.4,2.1 \mathrm{~Hz}, 1 \mathrm{H}), 6.86(\mathrm{~d}, J=2.1 \mathrm{~Hz}, 1 \mathrm{H}), 6.82(\mathrm{~d}, J=8.4 \mathrm{~Hz}, 1 \mathrm{H}), 4.91$ (hept, $J=6.3 \mathrm{~Hz}, 1 \mathrm{H}), 3.85$ (s, 3H), $3.38(\mathrm{~s}, 2 \mathrm{H}), 2.34(\mathrm{~s}, 3 \mathrm{H}), 1.15(\mathrm{~d}, J=6.3 \mathrm{~Hz}, 6 \mathrm{H})$. ${ }^{13} \mathrm{C}$ NMR $(150 \mathrm{MHz}) \delta=171.2,155.8,137.7,132.8,130.9,130.2,129.8,128.4,127.2$, 125.6, 110.7, 68.2, 56.1, 40.9, 21.8, 21.3. HR-MS(ESI) $\mathrm{m} / z$ calcd for: $\mathrm{C}_{19} \mathrm{H}_{23} \mathrm{O}_{3} \mathrm{~S}^{+}$ $\left[\mathrm{M}+\mathrm{H}^{+}\right]$331.1362, found 331.1359<smiles>COc1ccc(Sc2cc(OC)ccc2CC(=O)OC(C)C)cc1</smiles>

Isopropyl 2-(4-methoxy-2-((4-methoxyphenyl)thio)phenyl)acetate (3sm): The general procedure $\mathbf{B}$ was followed using isopropyl 2-(4-methoxyphenyl)acetate (1s) (52 $\mathrm{mg}, 0.25 \mathrm{mmol})$ and 1,2-bis(4-methoxyphenyl)disulfane $(\mathbf{2 m})(140.0 \mathrm{mg}, 0.5 \mathrm{mmol})$. Purification by column chromatography (Petroleum/EtOAc: $10 / 1, \mathrm{Rf}=0.56$ ) yielded 3sm (67 mg, 77\%) as a colorless oil. ${ }^{1} \mathrm{H}$ NMR (600 MHz) $\delta=7.42-7.40(\mathrm{~m}, 2 \mathrm{H}), 7.02$ $(\mathrm{dd}, J=8.3,1.8 \mathrm{~Hz}, 1 \mathrm{H}), 6.91-6.87(\mathrm{~m}, 2 \mathrm{H}), 6.79(\mathrm{~d}, J=8.3 \mathrm{~Hz}, 1 \mathrm{H}), 6.68(\mathrm{~d}, J=1.8$ Hz, 1H), 4.91 (hept, $J=6.2 \mathrm{~Hz}, 1 \mathrm{H}), 3.87$ (s, 3H), 3.81 (s, 3H), 3.36 (s, 2H), 1.14 (d, $J$ $=6.2 \mathrm{~Hz}, 6 \mathrm{H}) .{ }^{13} \mathrm{C}$ NMR $(150 \mathrm{MHz}) \delta=171.2,160.0,154.9,136.0,130.5,128.9,127.5$, 127.2, 122.7, 115.1, 110.5, 68.2, 56.0, 55.4, 40.9, 21.8. HR-MS(ESI) $\mathrm{m} / z$ calcd for: $\mathrm{C}_{19} \mathrm{H}_{23} \mathrm{O}_{4} \mathrm{~S}^{+}\left[\mathrm{M}+\mathrm{H}^{+}\right]$347.1312, found 347.1311<smiles>CCCCCCCCCC(=O)OC(C)C</smiles>

Isopropyl 2-(2-((4-chlorophenyl)thio)-4-methoxyphenyl)acetate (3sn): The general procedure B was followed using isopropyl 2-(4-methoxyphenyl)acetate (1s) (52 mg, $0.25 \mathrm{mmol}$ ) and 1,2-bis(4-chlorophenyl)disulfane (2n) (144.0 mg, $0.5 \mathrm{mmol})$. Purification by column chromatography (Petroleum/EtOAc: $10 / 1, \mathrm{Rf}=0.49$ ) yielded 3sn (55 mg, 63\%) as a colorless oil. ${ }^{1} \mathrm{H}$ NMR (600 MHz) $\delta=7.25-7.22(\mathrm{~m}, 2 \mathrm{H}), 7.21$ $-7.19(\mathrm{~m}, 2 \mathrm{H}), 7.18(\mathrm{dd}, J=8.4,2.2 \mathrm{~Hz}, 1 \mathrm{H}), 7.06(\mathrm{~d}, J=2.2 \mathrm{~Hz}, 1 \mathrm{H}), 6.86(\mathrm{~d}, J=8.4$ 
Hz, 1H), 4.96-4.94 (hept, $J=6.3 \mathrm{~Hz}, 1 \mathrm{H}), 3.82$ (s, 3H), 3.44 (s, 2H), 1.18 (d, $J=6.3$ $\mathrm{Hz}, 6 \mathrm{H}) .{ }^{13} \mathrm{C}$ NMR $(151 \mathrm{MHz}) \delta=171.1,156.9,133.7,133.4,132.9,131.9,129.9$, $129.3,127.4,122.9,111.2,68.4,56.1,40.7,21.8$. HR-MS(ESI) $\mathrm{m} / \mathrm{z}$ calcd for: $\mathrm{C}_{18} \mathrm{H}_{20} \mathrm{ClO}_{3} \mathrm{~S}^{+}\left[\mathrm{M}+\mathrm{H}^{+}\right]$351.0816, found 351.0812<smiles>Cc1ccc([Se]c2ccccc2)c(CC(=O)O)c1</smiles>

4

2-(5-methyl-2-(phenylselanyl)phenyl)acetic acid ${ }^{[2]}$ : The $N$-isopropyl-2-(5-methyl2-(phenylselanyl)phenyl)acetamide $(70 \mathrm{mg}, 0.2 \mathrm{mmol})$ dissolved in mixture of 1,4dioxane $(2 \mathrm{~mL})$ and $6 \mathrm{~N}$ aqueous $\mathrm{H}_{2} \mathrm{SO}_{4}(2 \mathrm{~mL})$. The reaction mixture was then heated at $105^{\circ} \mathrm{C}$. After $18 \mathrm{~h}$, heating was stopped and the solution was cooled to $\mathrm{rt} . \mathrm{H}_{2} \mathrm{O}(10$ $\mathrm{mL}$ ) was added to precipitate the product. The solid was isolated by filtration and dried in vacuo, Purification by column chromatography (Petroleum/EtOAc: $3 / 1, \mathrm{Rf}=0.30$ ) yielded 4 (48 mg, 79\%) as a white solid. M.p $=112-114{ }^{\circ} \mathrm{C} .{ }^{1} \mathrm{H}$ NMR $(600 \mathrm{MHz}) \delta=$ $7.49(\mathrm{~d}, J=7.9 \mathrm{~Hz}, 1 \mathrm{H}), 7.27-7.25(\mathrm{~m}, 2 \mathrm{H}), 7.20-7.15(\mathrm{~m}, 3 \mathrm{H}), 7.15(\mathrm{~s}, 1 \mathrm{H}), 7.03$ (d, $J=7.9 \mathrm{~Hz}, 1 \mathrm{H}), 3.85(\mathrm{~s}, 2 \mathrm{H}), 2.31(\mathrm{~s}, 3 \mathrm{H}) .{ }^{13} \mathrm{C} \mathrm{NMR}(150 \mathrm{MHz}) \delta=177.0,139.2,136.9$, $136.9,132.4,131.8,131.3,129.6,129.3,128.04,126.8,41.5,21.2$.<smiles>Cc1ccc([Se](=O)(=O)c2ccccc2)c(CC(=O)NC(C)C)c1</smiles>

5

$N$-isopropyl-2-(5-methyl-2-(phenylselenonyl)phenyl)acetamide ${ }^{[3]}:$ The $N$ isopropyl-2-(5-methyl-2-(phenylselanyl)phenyl) acetamide $(70 \mathrm{mg}, 0.2 \mathrm{mmol}$ ) dissolved in $2 \mathrm{~mL}$ acetic acid followed by $0.5 \mathrm{~mL} 35 \%$ hydrogen peroxide was added slowly. The reaction mixture was then heated at $50{ }^{\circ} \mathrm{C}$. After $1.5 \mathrm{~h}$, heating was stopped and the solution was cooled to $\mathrm{rt}$ and then the mixture poured into $5 \mathrm{~mL}$ ice-water, 
extracted with ethyl acetate $(3 \times 5 \mathrm{ml})$. The combined organic fractions were then washed with $\mathrm{H}_{2} \mathrm{O}(10 \mathrm{ml})$. The organic layer was dried with $\mathrm{Na}_{2} \mathrm{SO}_{4}$. Purification by column chromatography (DCE /MeOH: 10/1, Rf = 0.43) yielded $5(70 \mathrm{mg}, 93 \%)$ as a brown oil. ${ }^{1} \mathrm{H}$ NMR $(600 \mathrm{MHz}) \delta=7.71-7.69(\mathrm{~m}, 2 \mathrm{H}), 7.47(\mathrm{~d}, J=8.0 \mathrm{~Hz}, 1 \mathrm{H}), 7.44$ $-7.42(\mathrm{~m}, 3 \mathrm{H}), 7.20(\mathrm{~s}, 1 \mathrm{H}), 7.13(\mathrm{~d}, J=8.1 \mathrm{~Hz}, 1 \mathrm{H}), 3.95-3.87(\mathrm{~m}, 1 \mathrm{H}), 3.83(\mathrm{~d}, J=$ $11.8 \mathrm{~Hz}, 1 \mathrm{H}), 3.31(\mathrm{~d}, J=13.9 \mathrm{~Hz}, 1 \mathrm{H}), 2.31(\mathrm{~s}, 3 \mathrm{H}), 1.08$ (d, $J=6.4 \mathrm{~Hz}, 3 \mathrm{H}), 1.05$ (d, $J=6.7 \mathrm{~Hz}, 3 \mathrm{H}) .{ }^{13} \mathrm{C} \mathrm{NMR}(151 \mathrm{MHz}) \delta=169.1,142.8,141.4,138.8,136.9,132.6$, 131.2, 129.7, 129.3, 129.1, 127.1, 41.7, 40.7, 22.6, 22.5, 21.4. HR-MS(ESI) $\mathrm{m} / z$ calcd for: $\mathrm{C}_{19} \mathrm{H}_{22} \mathrm{NO}_{3}{ }^{80} \mathrm{Se}^{+}\left[\mathrm{M}+\mathrm{H}^{+}\right] 380.0759$, found 380.0750

\section{Scale-up experiment of phenylacetamide 1i and diphenyl diselenide 2a}

A suspension of $N$-isopropyl-2-( $m$-tolyl) acetamide (1i) (960 mg, $5.0 \mathrm{mmol})$, diphenyl diselenide (2a) (3.12 g, $10.0 \mathrm{mmol}),\left[\mathrm{RuCl}_{2} \text { (p-cymene) }\right]_{2}$ (146 mg, $5.0 \mathrm{~mol} \%$ ), $\mathrm{CF}_{3} \mathrm{SO}_{3} \mathrm{H}$ (440 ul, $5.0 \mathrm{mmol}$ ), AgOTf (256 mg, $20 \mathrm{~mol} \%$ ), AdmCO ${ }_{2} \mathrm{H}$ (900.0 mg, 1.0 equiv) and $\mathrm{AgOPiv}(2.06 \mathrm{~g}, 10.0 \mathrm{mmol})$ in $\mathrm{CF}_{3} \mathrm{CH}_{2} \mathrm{OH}(10.0 \mathrm{~mL})$ was stirred under argon at $100{ }^{\circ} \mathrm{C}$ for $16 \mathrm{~h}$. At ambient temperature, the reaction mixture was quench with $\mathrm{H}_{2} \mathrm{O}(30 \mathrm{~mL})$ and extracted with EtOAc $(3 \times 50 \mathrm{~mL})$. The combined organic layers were dried over anhydrous $\mathrm{Na}_{2} \mathrm{SO}_{4}$. After filtration and evaporation of the solvents in vacuo, the crude product was purified by column chromatography on silica gel (Petroleum/DCE: $2: 1 \rightarrow$ Petroleum/EtOAc: $4 / 1)$ to afford the desired product 3ia (1126 mg, 65\%).

\section{Intermolecular competition between different substituted phenylacetamide 1}


<smiles>Cc1ccc(CC(=O)NC(C)C)cc1</smiles>

$1 \mathrm{a}$

$+$<smiles>CC(C)NC(=O)Cc1ccc(Br)cc1</smiles>

$1 \mathrm{p}$
$\mathrm{PhSeSePh}$ $\left[\mathrm{RuCl}_{2}(p \text {-cymene })\right]_{2}$ $\operatorname{AgOTf}(20 \mathrm{~mol} \%)$ AgOPiv (2.0 equiv) $\mathrm{CF}_{3} \mathrm{SO}_{3} \mathrm{H}$ (1.0 equiv) $\mathrm{AdmCO}_{2} \mathrm{H}$ (1.0 equiv) TFE, $100{ }^{\circ} \mathrm{C}, 16 \mathrm{~h}$ 3aa:3pa $=2.86: 1$

(by LC-MS)<smiles>Cc1ccc(CC(=O)NC(C)C)c([SeH]c2ccccc2)c1</smiles>

$+$<smiles>CC(C)NC(=O)Cc1ccc(Br)cc1[Se]c1ccccc1</smiles>

3 pa

The general procedure A was followed using $N$-isopropyl-2-(p-tolyl)acetamide (1a) (48 mg, $0.25 \mathrm{mmol}$ ), 2-(4-bromophenyl)- $N$-isopropylacetamide (1p) (68.3 mg, $0.25 \mathrm{mmol}$ ), 1,2-diphenyldiselane (2a) $(78.8 \mathrm{mg}, 0.25 \mathrm{mmol}),\left[\mathrm{RuCl}_{2}(p \text {-cymene) }]_{2}(7.9 \mathrm{mg}, 5.0\right.$ mol \%), $\mathrm{CF}_{3} \mathrm{SO}_{3} \mathrm{H}$ (20 ul, $\left.0.25 \mathrm{mmol}\right), \operatorname{AgOTf}(13 \mathrm{mg}, 20 \mathrm{~mol} \%), \mathrm{AdmCO}_{2} \mathrm{H}(41 \mathrm{mg}$, $0.25 \mathrm{mmol}$ ), AgOPiv (103 mg, 2.0 equiv) in TFE ( $2 \mathrm{ml}$ ) was stirred under argon at $100{ }^{\circ} \mathrm{C}$ for $16 \mathrm{~h}$. The ratio of 3aa:3pa $=2.86: 1$ which was estimated by LC-MS.

\section{Ruthenium-Catalyzed H/D Exchange of 1a with $\mathrm{CD}_{3} \mathrm{OD}$ as Cosolvent}<smiles>Cc1ccc(CC(=O)NC(C)C)c(CC(=O)NC(C)C)c1</smiles>

The general procedure A was followed using $N$-isopropyl-2-(p-tolyl)acetamide (1a) (48 $\mathrm{mg}, 0.25 \mathrm{mmol}),\left[\mathrm{RuCl}_{2} \text { (p-cymene) }\right]_{2}(7.9 \mathrm{mg}, 5.0 \mathrm{~mol} \%), \mathrm{CF}_{3} \mathrm{SO}_{3} \mathrm{H}$ (20 ul, 1.0 equiv), AgOTf (13 mg, $20 \mathrm{~mol} \%$ ), AdmCO $2 \mathrm{H}$ (41 mg, $0.25 \mathrm{mmol}$ ), AgOPiv (103 mg, 2.0 equiv) in a solvent mixture of $\mathrm{CF}_{3} \mathrm{CH}_{2} \mathrm{OH}$ and $\mathrm{CD}_{3} \mathrm{OD}(1.8 / 0.2 \mathrm{~mL})$ under argon at $100{ }^{\circ} \mathrm{C}$ for $16 \mathrm{~h}$. Purification by column chromatography (Petroleum/DCE: 2/1 $\rightarrow$ Petroleum/EtOAc: $5 / 1$ ) yielded $[\mathrm{D}]_{\mathrm{n}}-\mathbf{1 a}(45 \mathrm{mg}, 94 \%)$ as pale solid. The Dincorporation in $[D]_{\mathrm{n}}-\mathbf{1 a}$ was estimated by ${ }^{1} \mathrm{H}-\mathrm{NMR}$ spectroscopy 


\section{Control experiment in the presence of TEMPO}

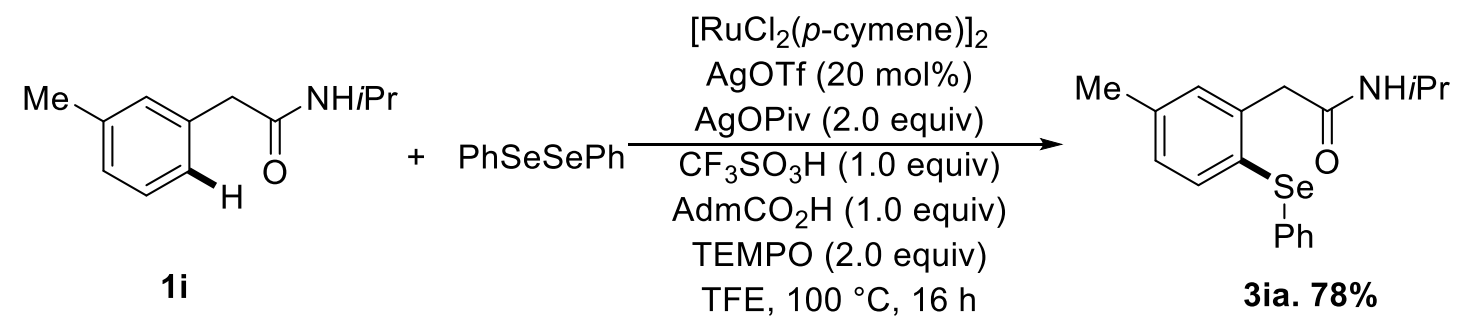

The general procedure A was followed using $N$-isopropyl-2-( $p$-tolyl)acetamide (1i) (48 mg, $0.25 \mathrm{mmol}$ ), 1,2-diphenyldiselane (2a) (78.8 mg, $0.25 \mathrm{mmol}),\left[\mathrm{RuCl}_{2} \text { (p-cymene) }\right]_{2}$ (7.9 mg, $5.0 \mathrm{~mol} \%$ ), $\mathrm{CF}_{3} \mathrm{SO}_{3} \mathrm{H}$ (20ul, $0.25 \mathrm{mmol}$ ), AgOTf (13 mg, $20 \mathrm{~mol} \%$ ), $\mathrm{AdmCO}_{2} \mathrm{H}$ (41mg, $0.25 \mathrm{mmol}$ ), AgOPiv (103 mg, 2.0 equiv), the radical scavenger TEMPO (2.0 equiv. $0.5 \mathrm{mmol}$ ) were added. Purification by column chromatography (Petroleum/DCE:2/1 $\rightarrow$ Petroleum/EtOAc: $4 / 1$ ) yielded 3ia (67 mg, 78\%) as a white solid

\section{Reference:}

(1) Rolfe, A.; Probst, D. A.; Volp, K. A.; Omar, I.; Flynn, D. L.; Hanson, P. R. J. Org. Chem. 2008, 73, 8785-8790.

(2) Sarabu, R.; Bizzarro, F. T.; Corbett, W. L.; Dvorozniak, M. T.; Geng, W.; Grippo, J. F.; Haynes, N.-E.; Hutchings, S.; Garofalo, L.; Guertin, K. R.; Hilliard, D. W.; Kabat, M.; Kester, R. F.; Ka, W.; Liang, Z.; Mahaney, P. E.; Marcus, L.; Matschinsky, F. M.; Moore, D.; Racha, J.; Radinov, R.; Ren, Y.; Qi, L.; Pignatello, M.; Spence, C. L.; Steele, T.; Tengi, J.; Grimsby, J. J. Med. Chem. 2012, 55, 7021-7036.

(3) Wang, X.; Zhao, L.; Shao, S.; Ding, J.; Wang, L.; Jing, X.; Wang, F. Macromolecules. 2014, 47, 2907-2914. 
<smiles>Cc1ccc(CC(=O)NC(C)C)c([Se]c2ccccc2)c1</smiles>

3 aa

(CDCl ${ }_{3}, 600 \mathrm{MHz}$ )

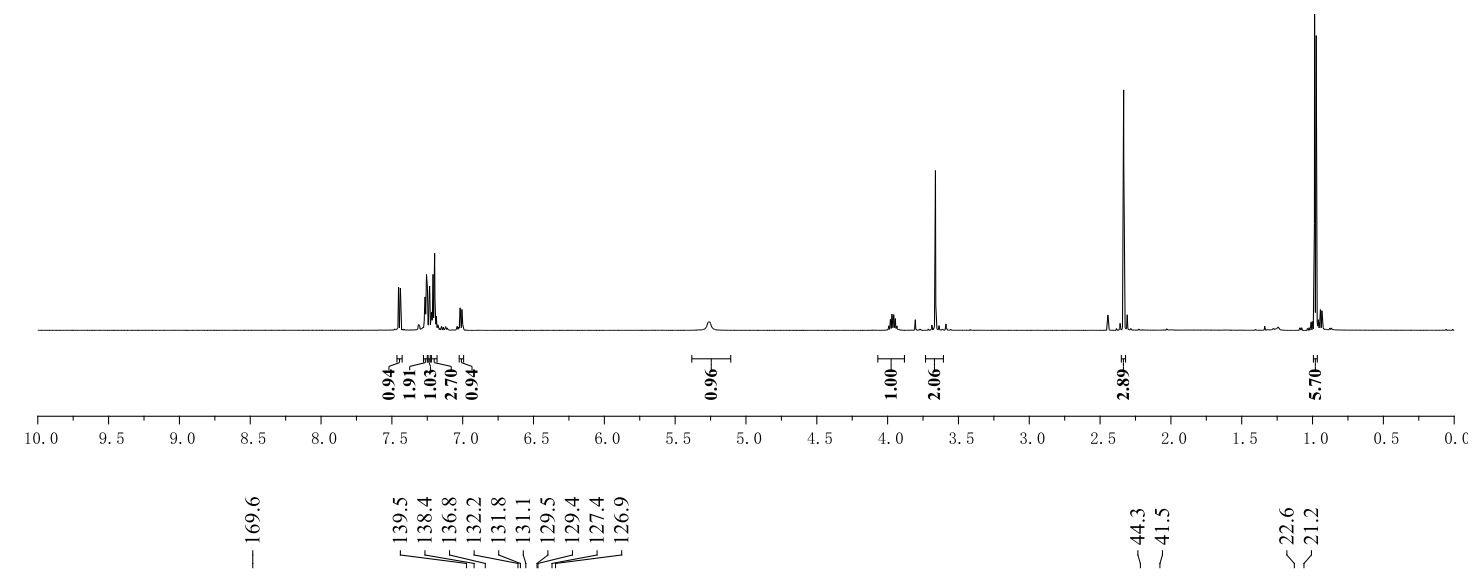<smiles>Cc1ccc(CC(=O)NC(C)C)c([Se]c2ccccc2)c1</smiles>

3 aa

$\left(\mathrm{CDCl}_{3}, 150 \mathrm{MHz}\right)$
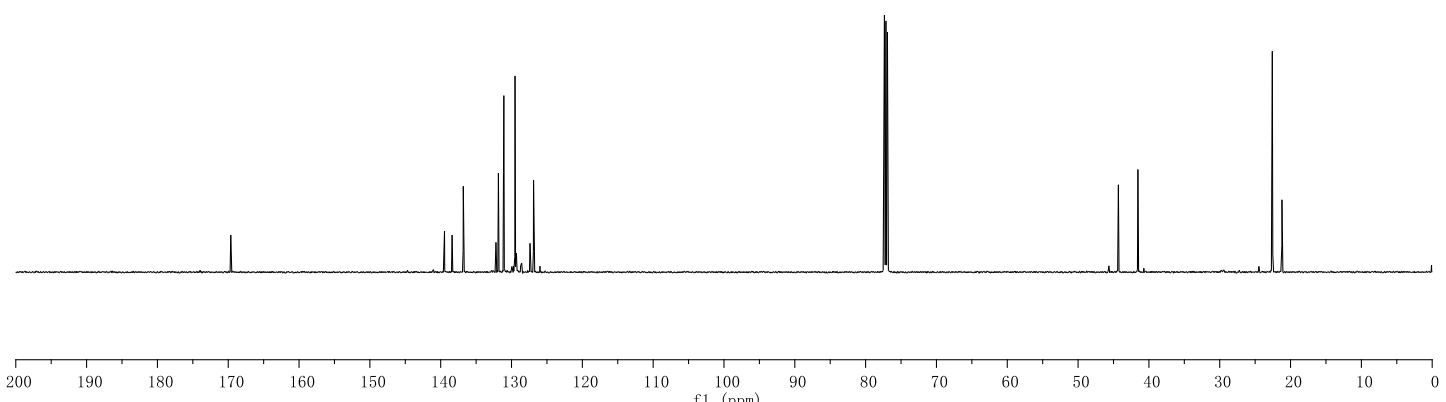
<smiles>CCNC(=O)Cc1ccc(C)cc1[Se]c1ccccc1</smiles>

3 ba

$(\mathrm{CDCl} 3,600 \mathrm{MHz})$

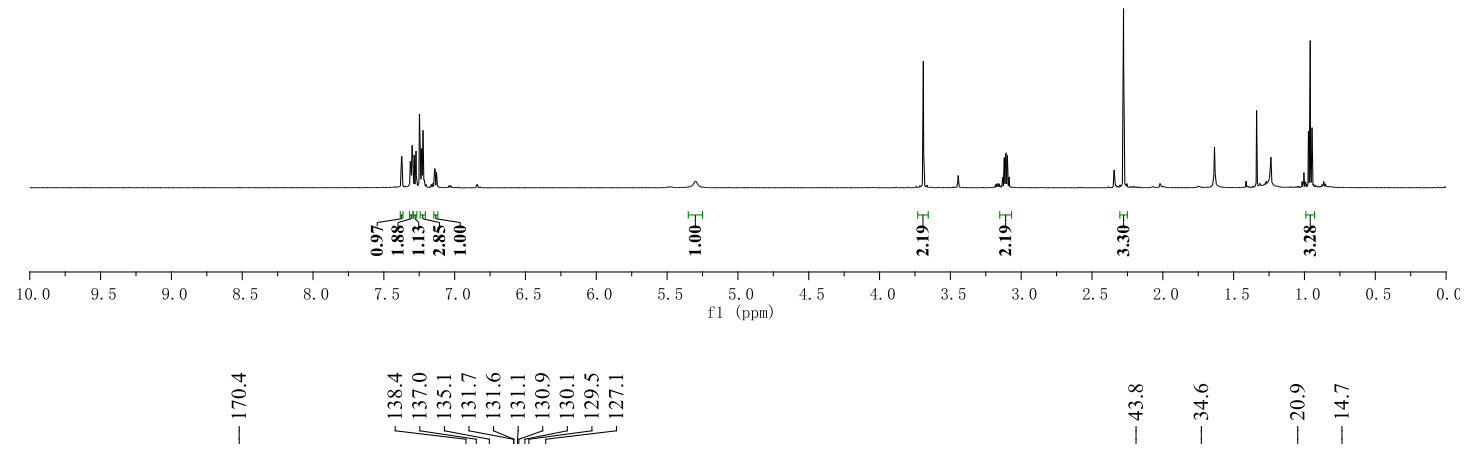<smiles>CCNC(=O)Cc1ccc(C)cc1[Se]c1ccccc1</smiles>

3 ba

(CDCl3, $150 \mathrm{MHz}$ )

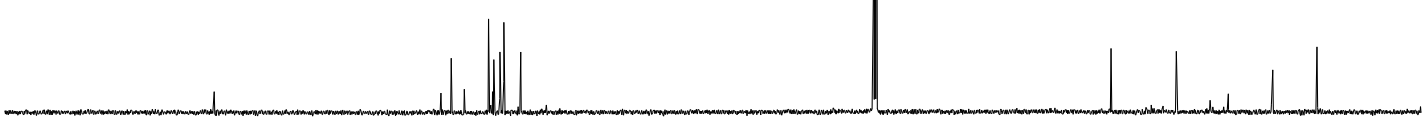

\begin{tabular}{llllllllllllllllllllll}
\hline 200 & 190 & 180 & 170 & 160 & 150 & 140 & 130 & 120 & 110 & 100 & 90 & 80 & 70 & 60 & 50 & 40 & 30 & 10 & 10 & 1 \\
\hline
\end{tabular} 

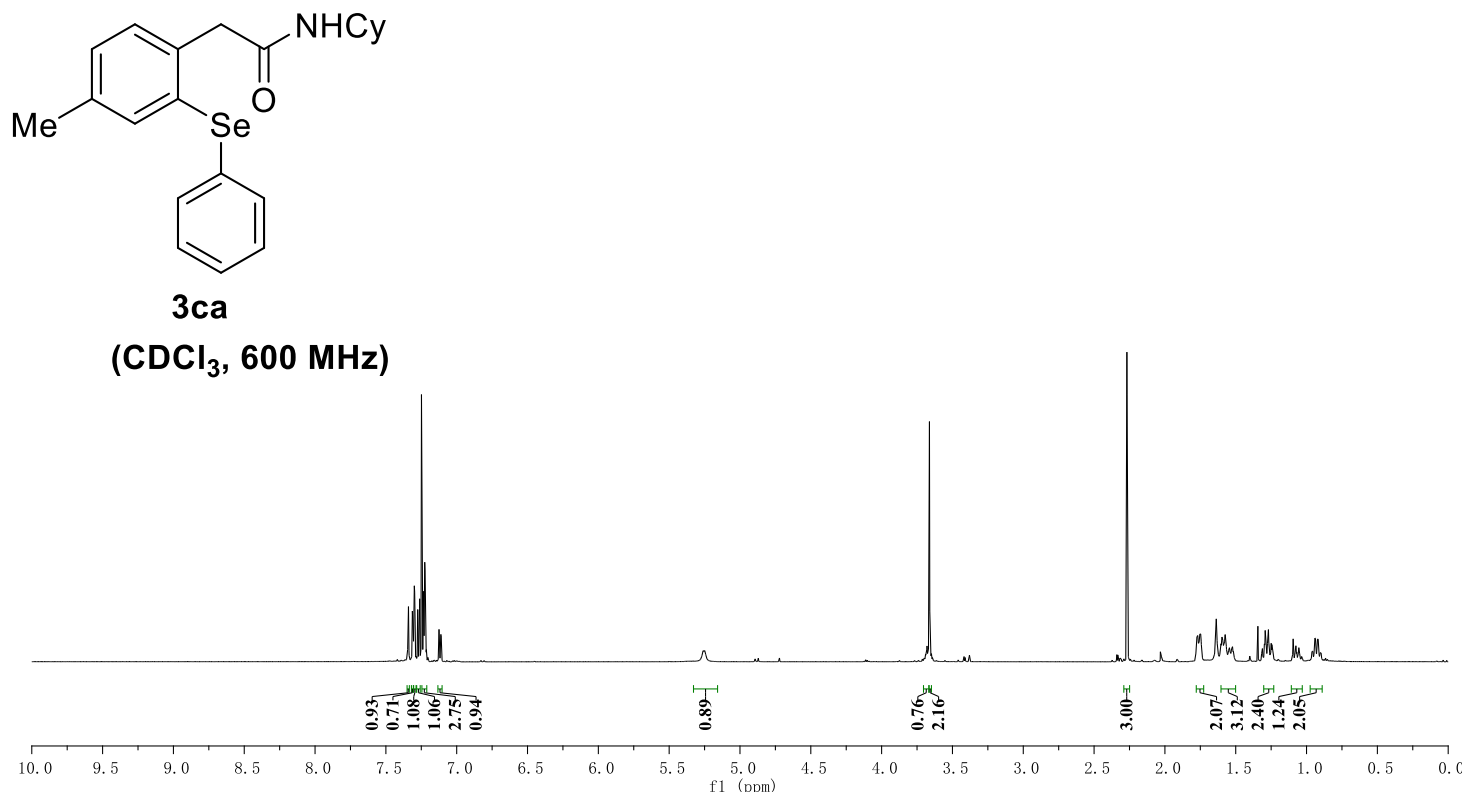

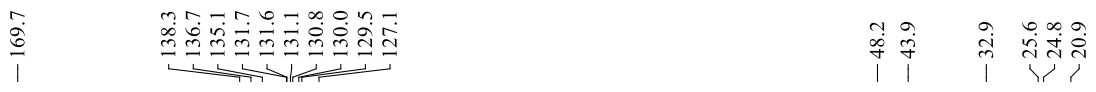

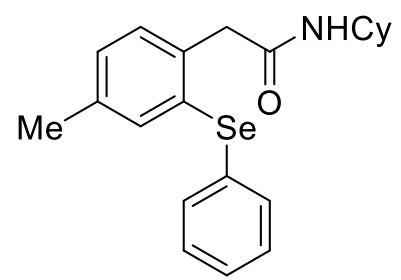

$3 \mathrm{ca}$

$\left(\mathrm{CDCl}_{3}, 150 \mathrm{MHz}\right)$

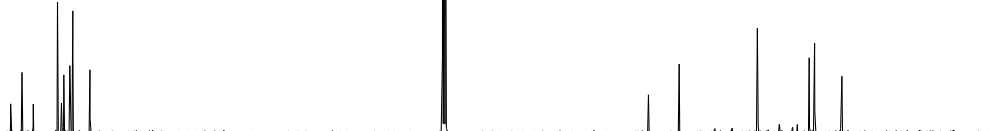

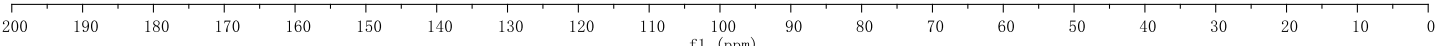


<smiles>Cc1ccc(CC(=O)NCC(C)C)c([Se]c2ccccc2)c1</smiles>

3 da

$\left(\mathrm{CDCl}_{3}, 600 \mathrm{MHz}\right)$

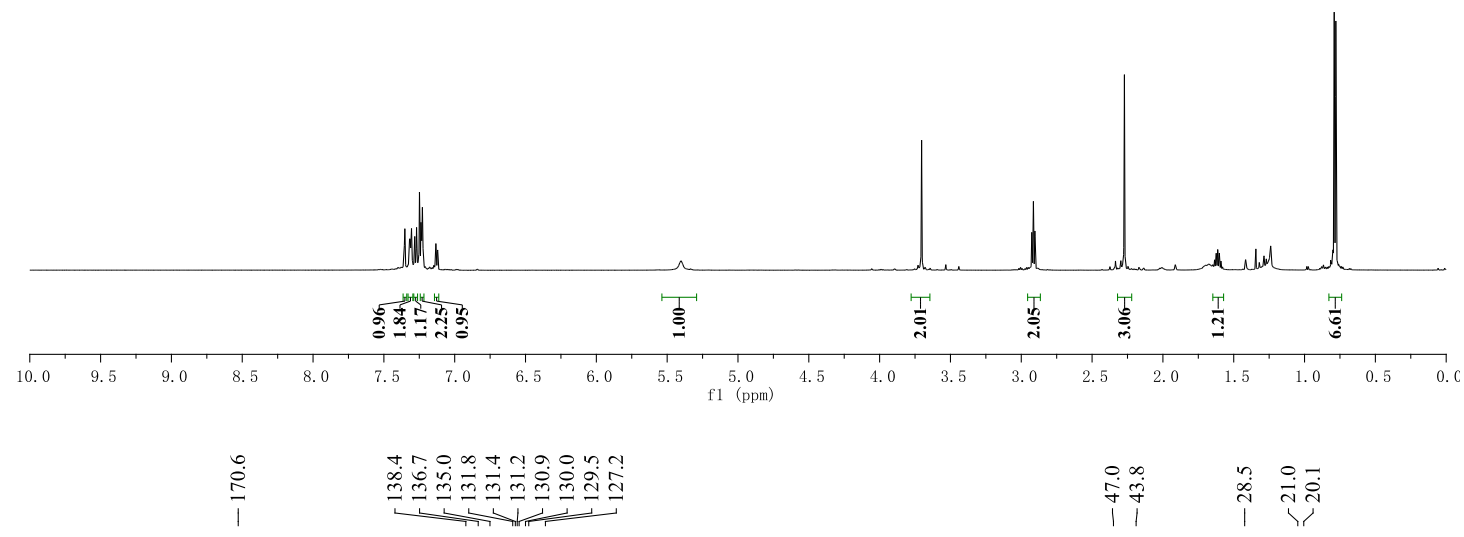<smiles>Cc1ccc(CC(=O)NCC(C)C)c([Se]c2ccccc2)c1</smiles>

3 da

$\left(\mathrm{CDCl}_{3}, 150 \mathrm{MHz}\right)$

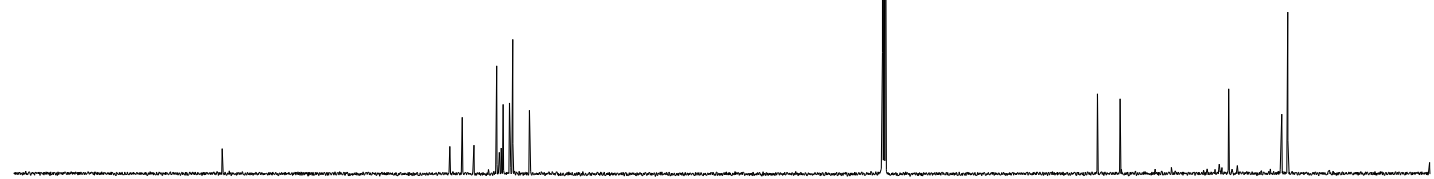

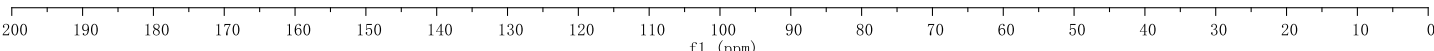


<smiles>Cc1ccc(CC(=O)NC(C)C(=O)OC(C)C)c([Se]c2ccccc2)c1</smiles>

3ea

$\left(\mathrm{CDCl}_{3}, 600 \mathrm{MHz}\right)$
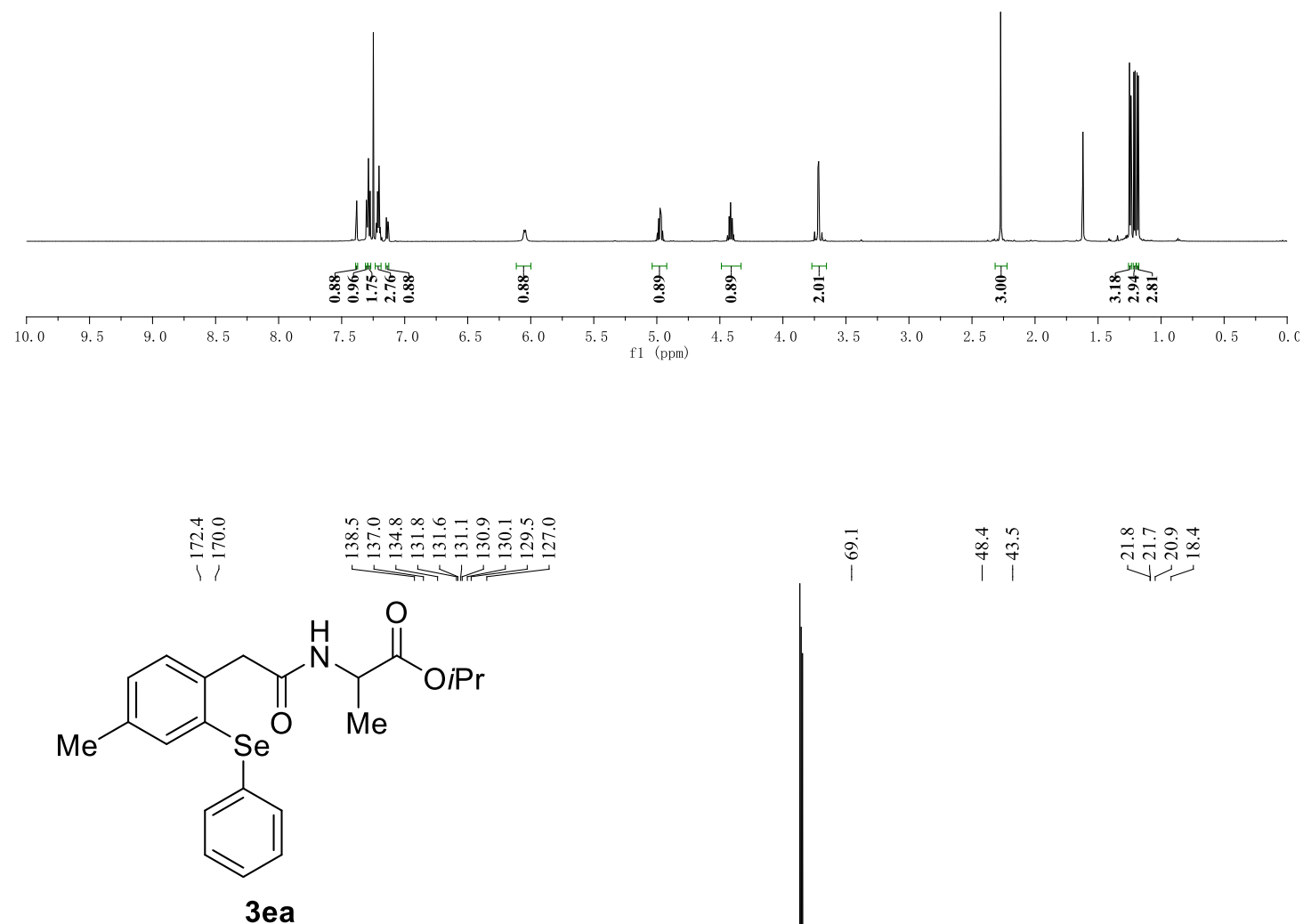

$\left(\mathrm{CDCl}_{3}, 150 \mathrm{MHz}\right)$

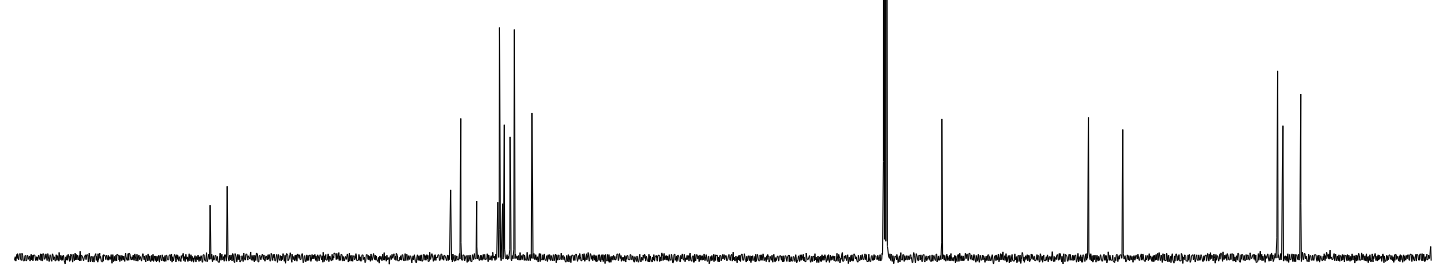

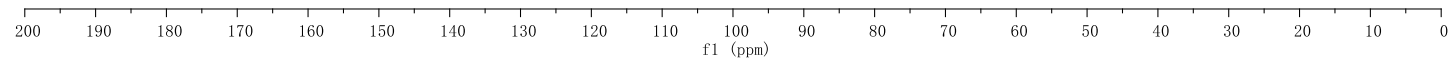


<smiles>Cc1ccc(CC(=O)N2CCCCC2)c([Se]c2ccccc2)c1</smiles>

$3 \mathrm{fa}$

$\left(\mathrm{CDCl}_{3}, 600 \mathrm{MHz}\right)$

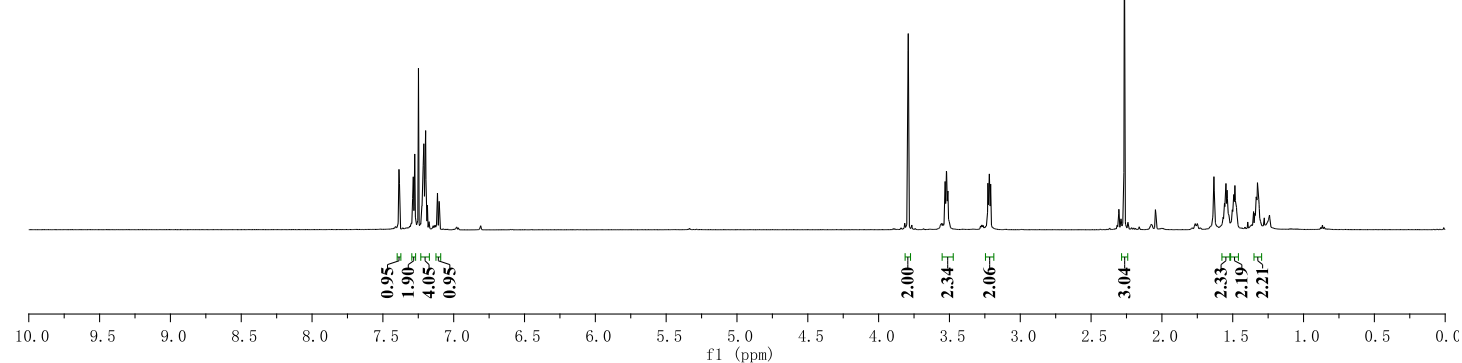

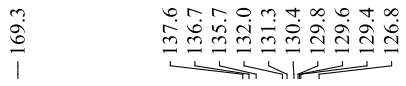

1)<smiles>Cc1ccc(CC(=O)N2CCCCC2)c([Se]c2ccccc2)c1</smiles>

$3 \mathrm{fa}$

$\left(\mathrm{CDCl}_{3}, 150 \mathrm{MHz}\right)$

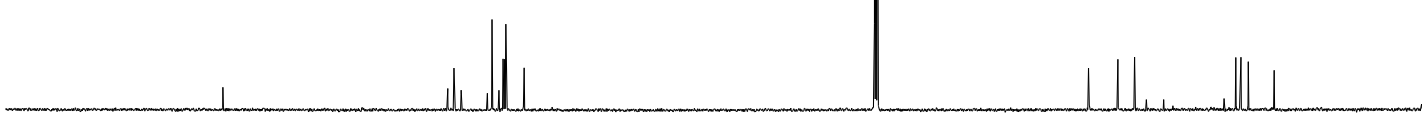

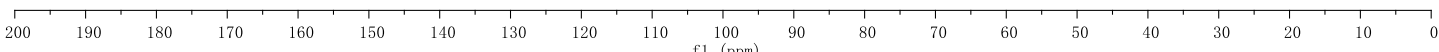


<smiles>NC(=O)Cc1ccccc1[Se]c1ccccc1</smiles>

3ga

$\left(\mathrm{CDCl}_{3}, 600 \mathrm{MHz}\right)$

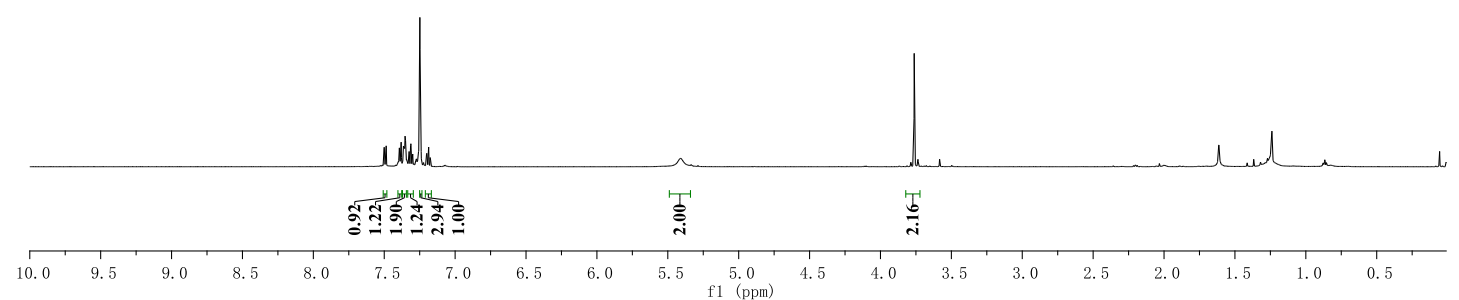

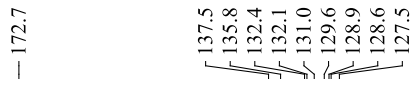<smiles>NC(=O)Cc1ccccc1[Se]c1ccccc1</smiles>

3 ga

$\left(\mathrm{CDCl}_{3}, 150 \mathrm{MHz}\right)$

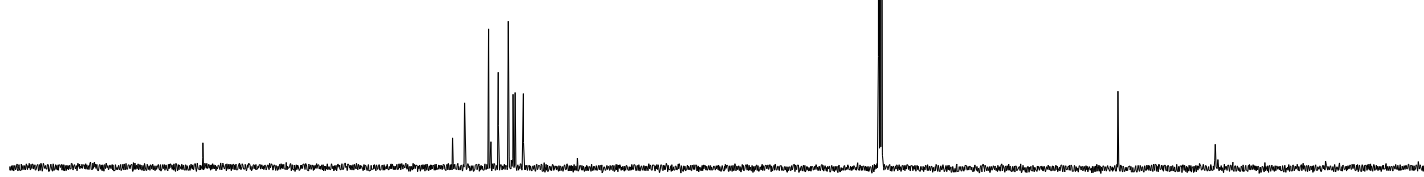

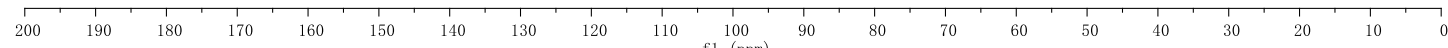


<smiles>CC(C)NC(=O)CCc1ccccc1Oc1ccccc1</smiles>

3ha

$\left(\mathrm{CDCl}_{3}, 600 \mathrm{MHz}\right)$

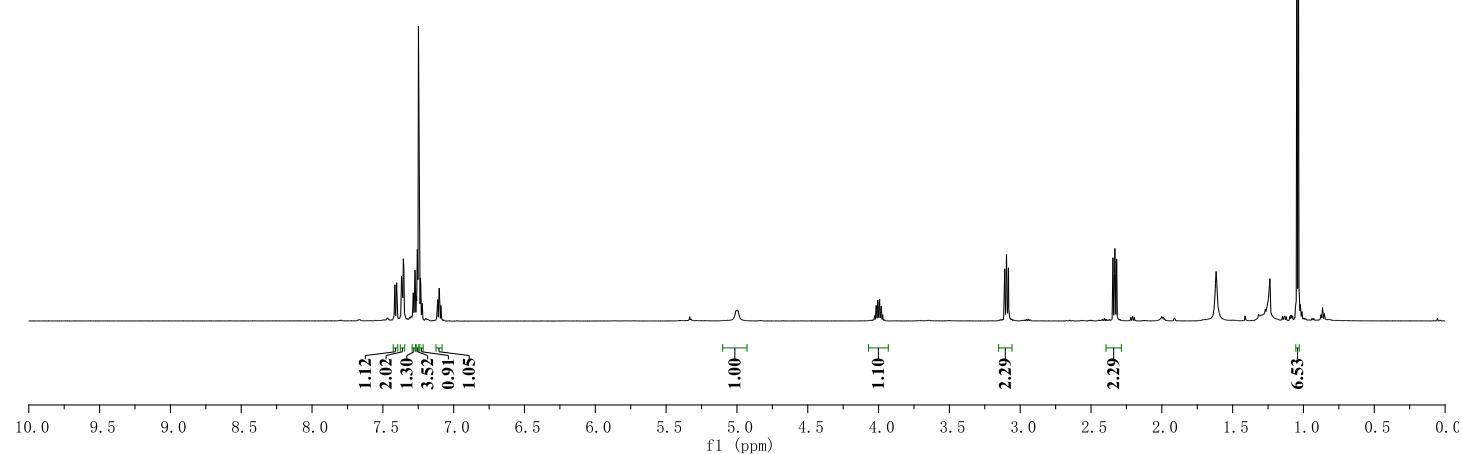

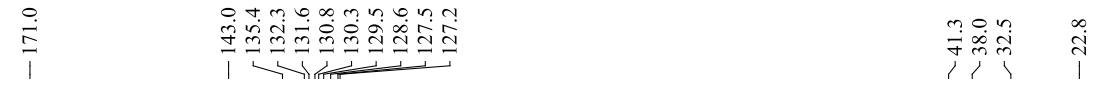<smiles>CCCNC(=O)CCc1ccccc1[Se]c1ccccc1</smiles>

(CDCl $3,150 \mathrm{MHz}$ )

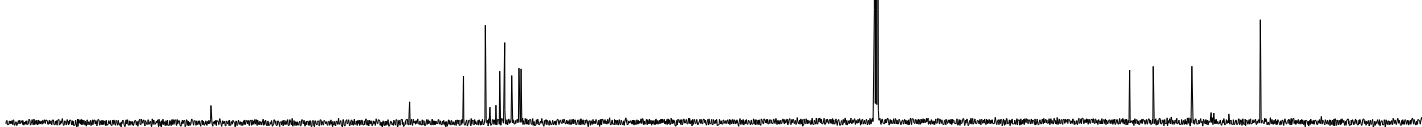

\begin{tabular}{lllllllllllllllllllll}
\hline 200 & 190 & 180 & 170 & 160 & 150 & 140 & 130 & 120 & 110 & 100 & 90 & 80 & 70 & 60 & 50 & 40 & 30 & 20 & 10 & 0 \\
\hline
\end{tabular} 


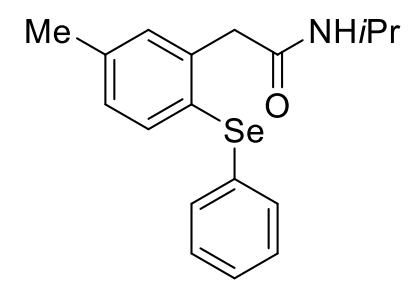

3 ia

$\left(\mathrm{CDCl}_{3}, 600 \mathrm{MHz}\right)$

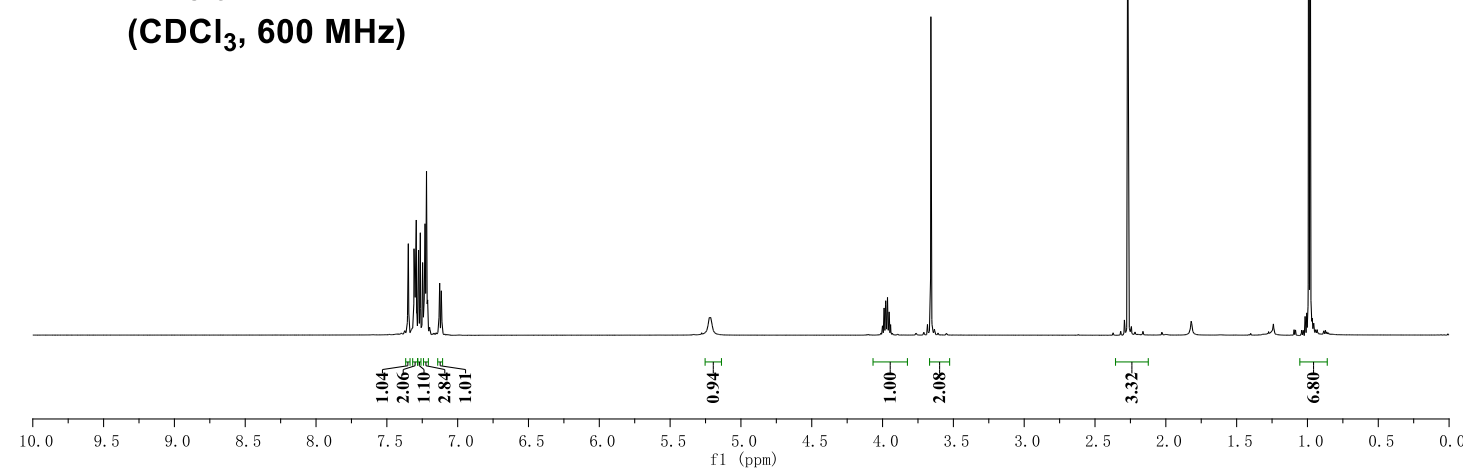

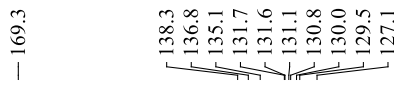<smiles>CCCNC(=O)Cc1cc(C)ccc1Oc1ccccc1</smiles>

3 ia

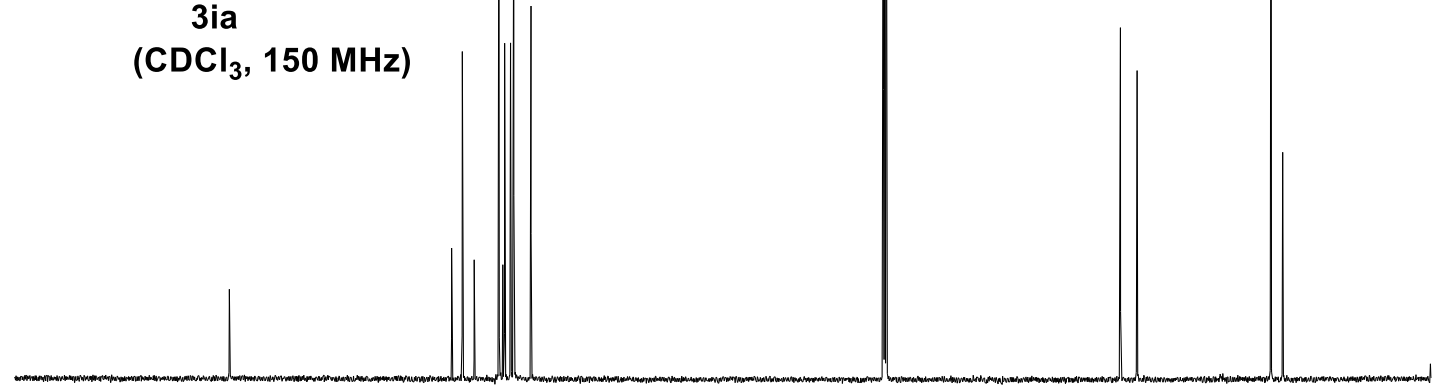

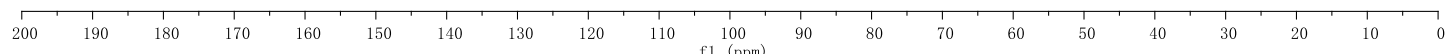


<smiles>CCCNC(=O)Cc1cc(OC)ccc1Oc1ccccc1</smiles>

3ja (CDCl $3,600 \mathrm{MHz}$ )

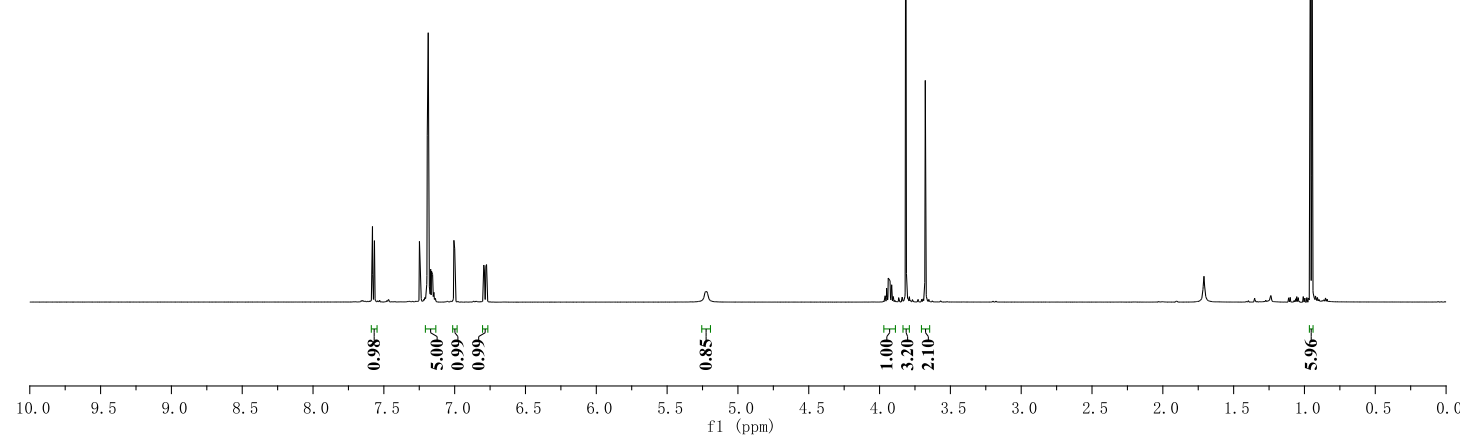

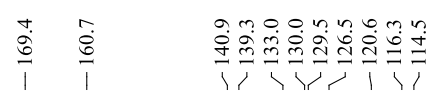<smiles>COc1ccc([Se]c2ccccc2)c(CC(=O)NC(C)C)c1</smiles>

3 ja $\left(\mathrm{CDCl}_{3}, 150 \mathrm{MHz}\right)$
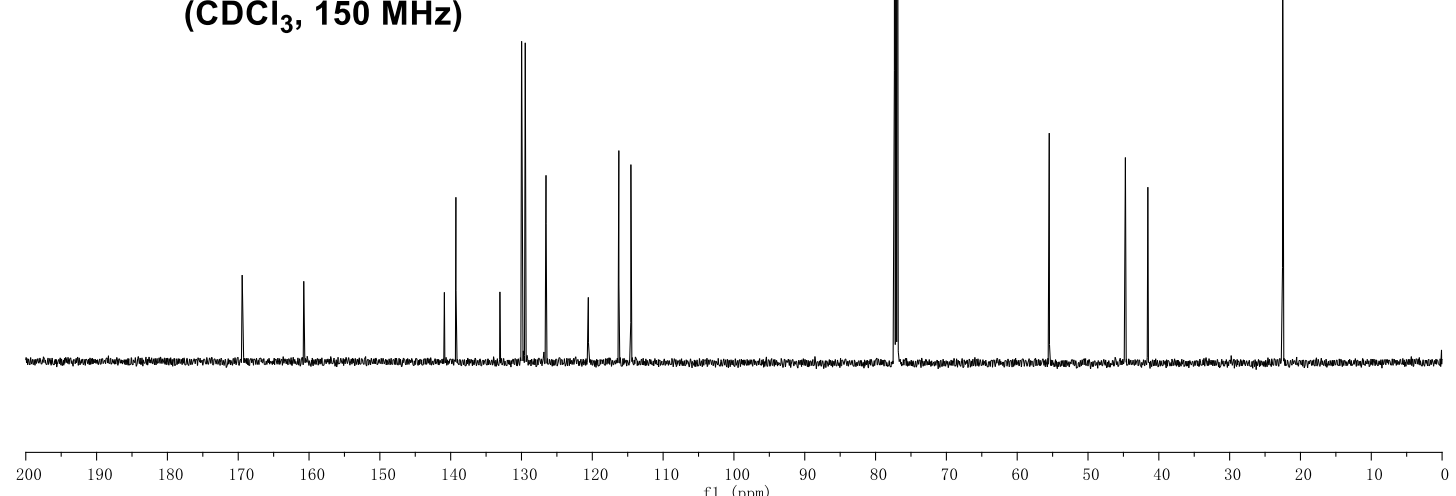


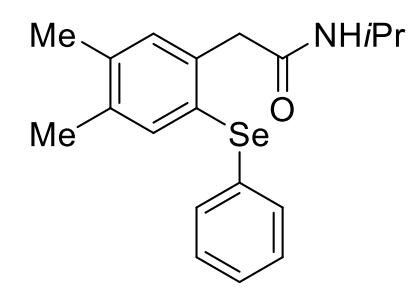

3 ka

(CDCl, $600 \mathrm{MHz}$ )

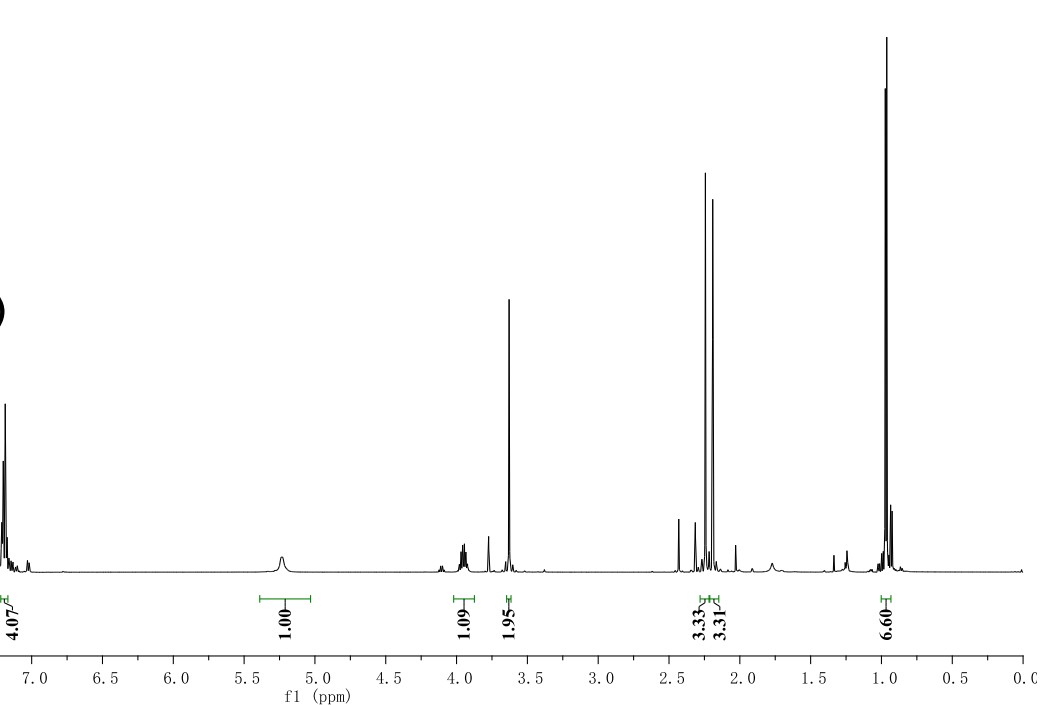

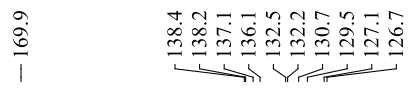

भु?<smiles>Cc1cc(CC(=O)NC(C)C)c([Se]c2ccccc2)cc1C</smiles>

3 ka

$\left(\mathrm{CDCl}_{3}, 150 \mathrm{MHz}\right)$

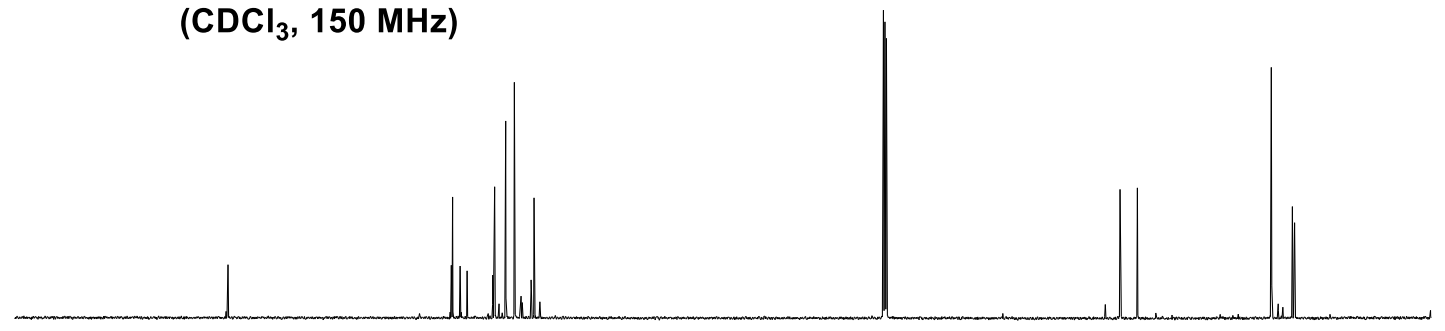

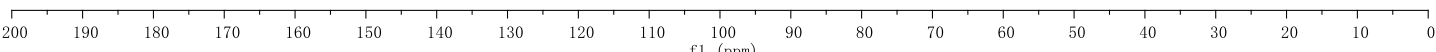




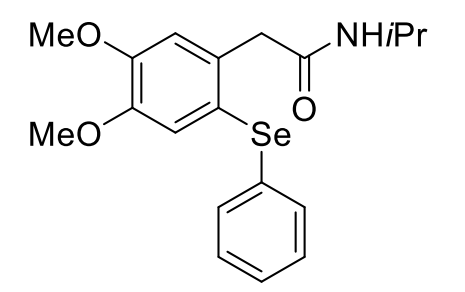

$31 \mathrm{a}$

$\left(\mathrm{CDCl}_{3}, 600 \mathrm{MHz}\right)$

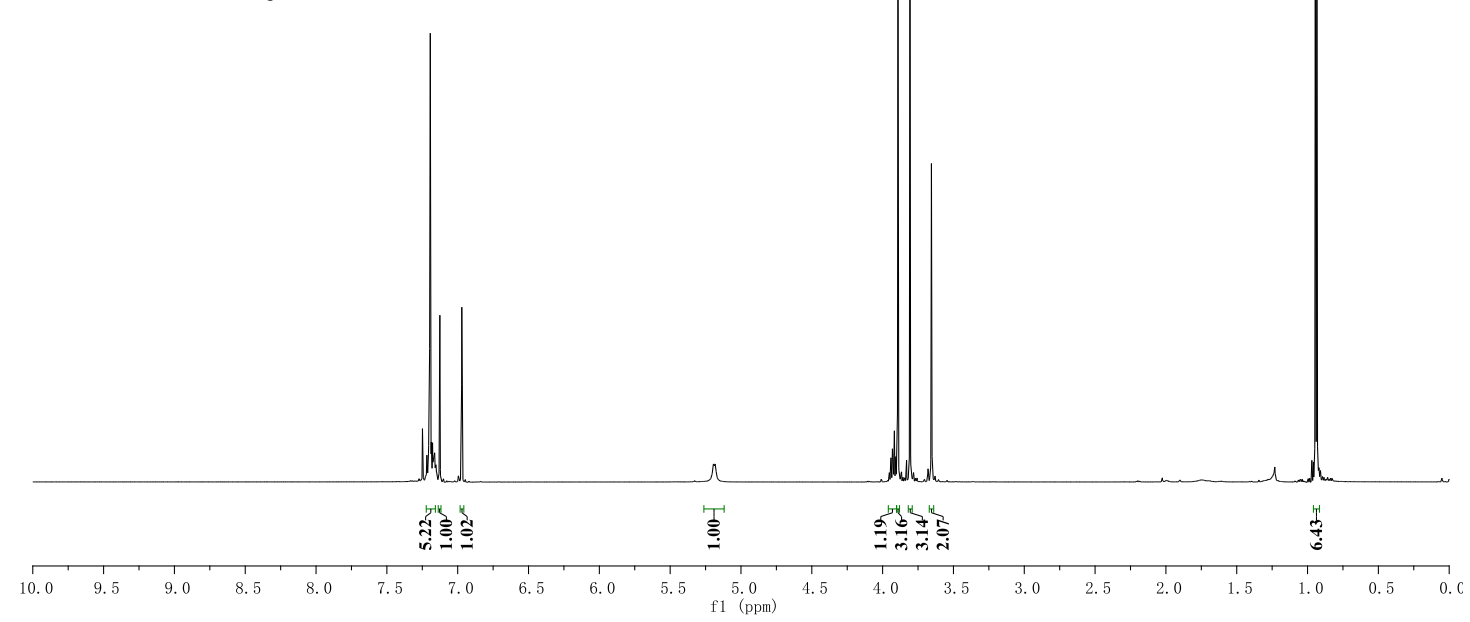

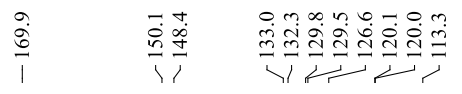

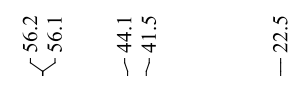

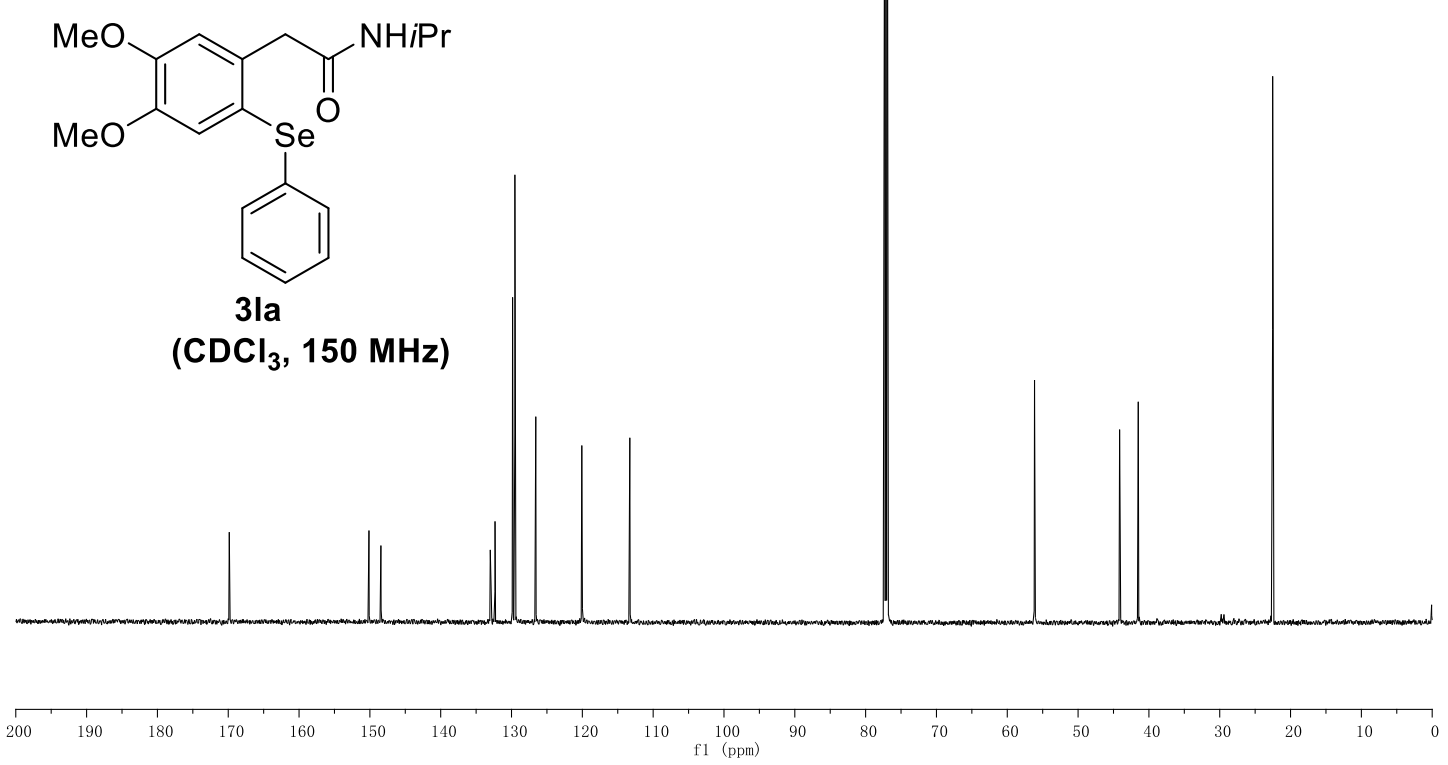




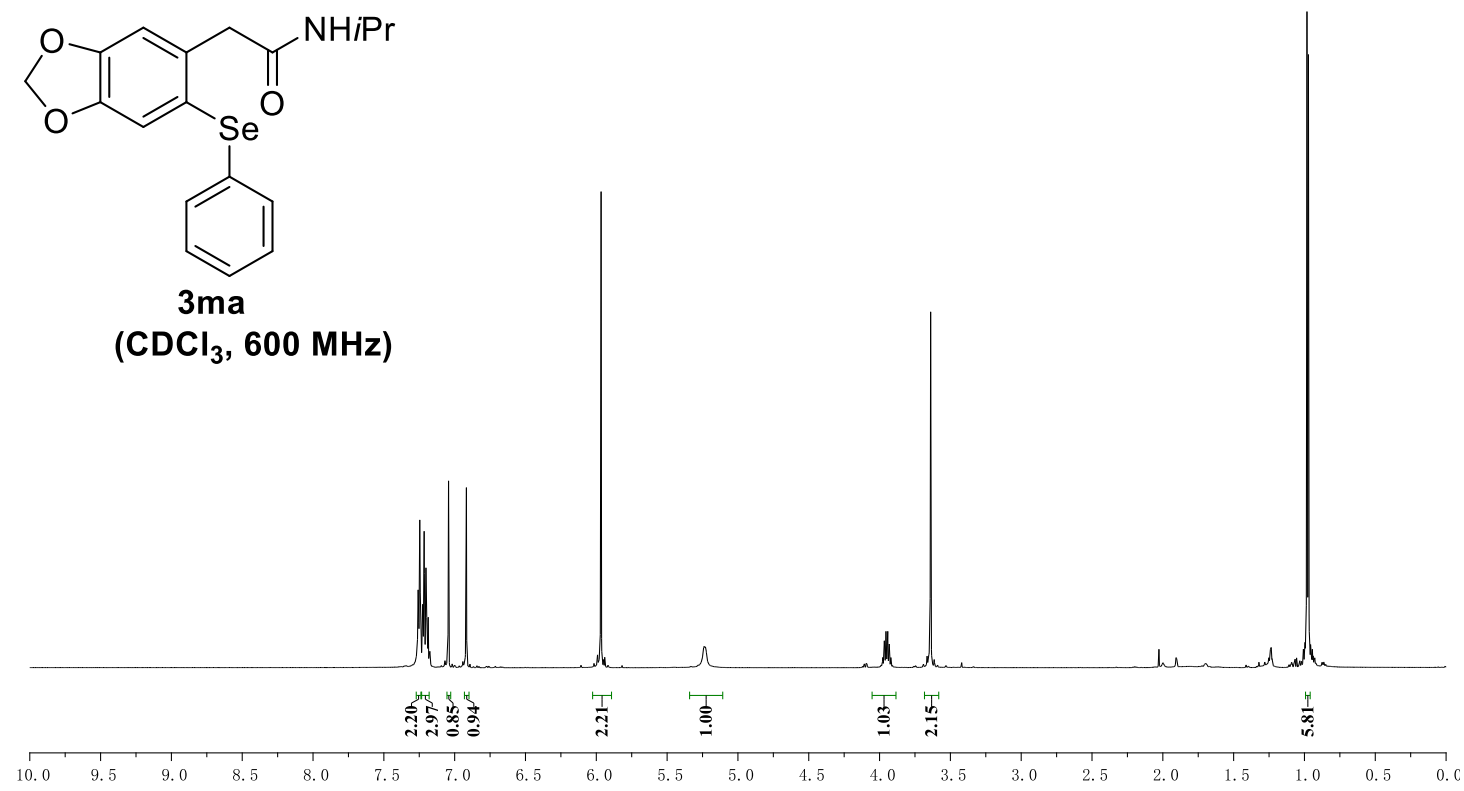

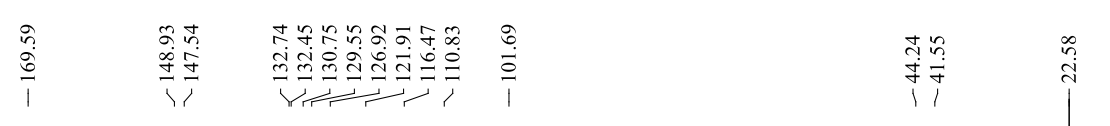

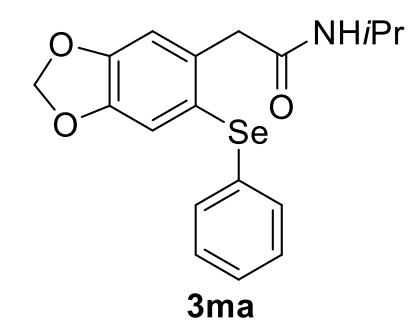

(CDCl $3,150 \mathrm{MHz}$ )

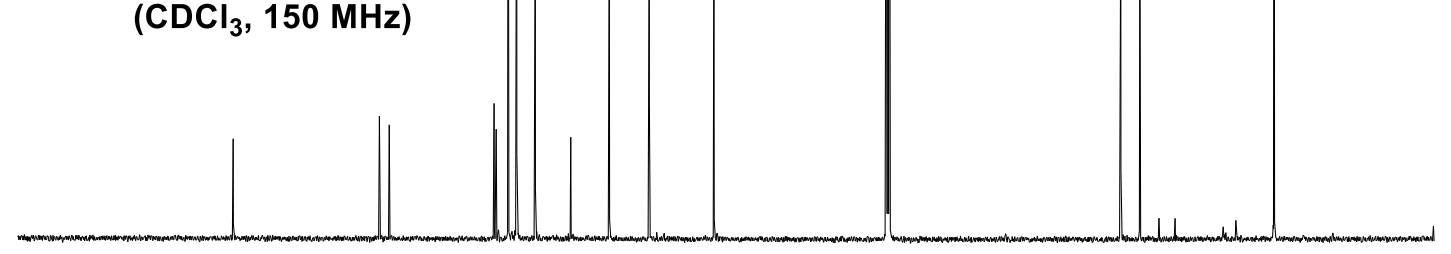

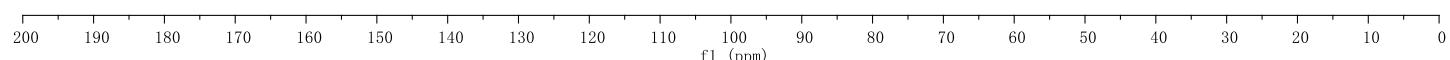


<smiles>CC(C)NC(=O)Cc1ccccc1[Se]c1ccccc1</smiles>

3na

$\left(\mathrm{CDCl}_{3}, 600 \mathrm{MHz}\right)$

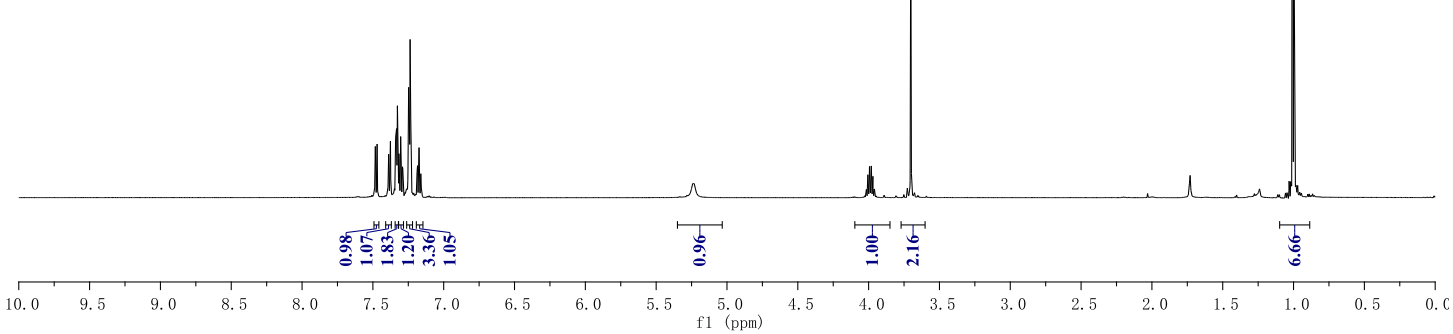

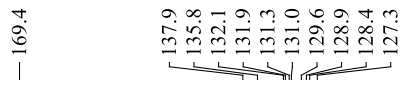<smiles>CC(C)NC(=O)Cc1ccccc1[Se]c1ccccc1</smiles>

3na

$\left(\mathrm{CDCl}_{3}, 150 \mathrm{MHz}\right)$

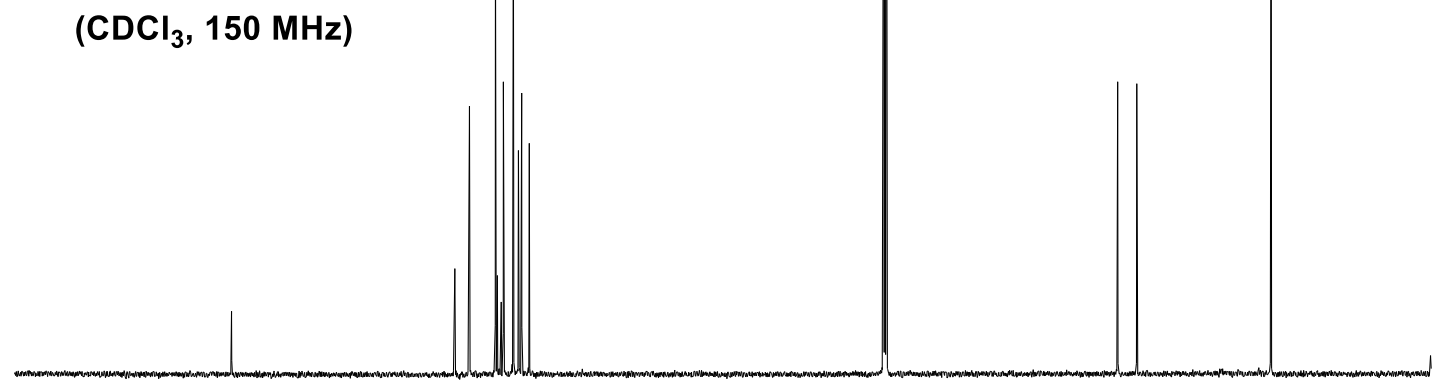

200
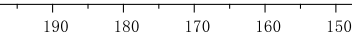

$\underset{\substack{1 \\ j}}{\substack{4 \\ 0}}$

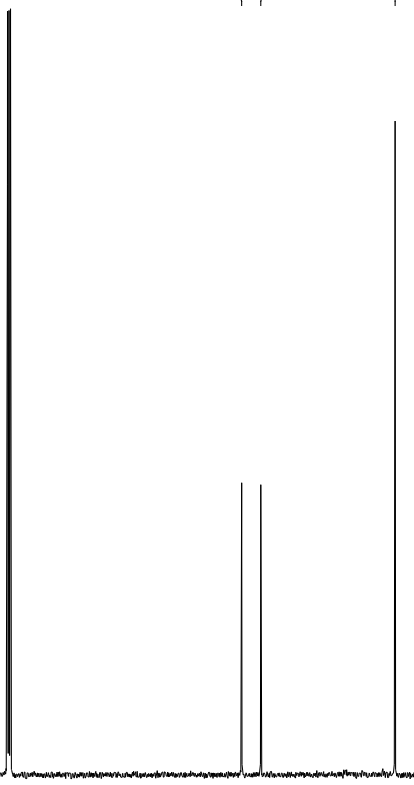


<smiles>CCCNC(=O)Cc1ccc(C(C)(C)C)cc1[Se]c1ccccc1</smiles>

30 a

$\left(\mathrm{CDCl}_{3}, 600 \mathrm{MHz}\right)$

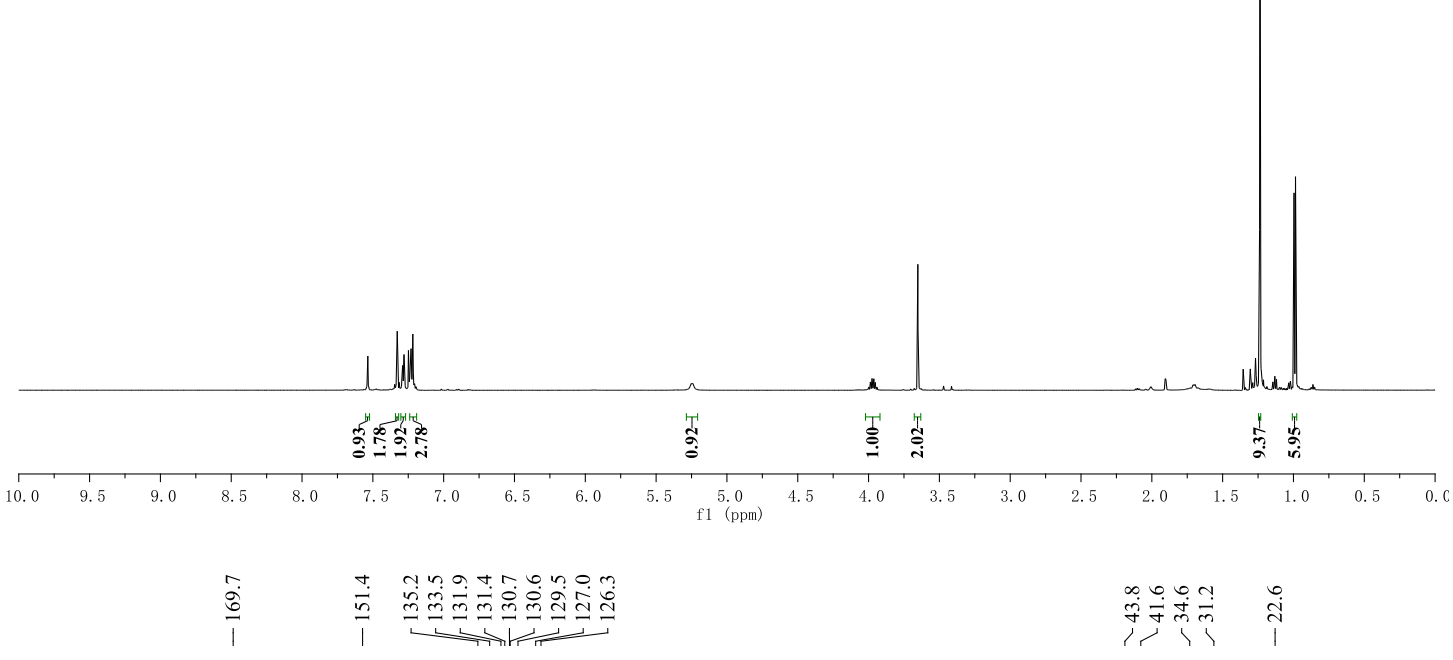<smiles>CCCNC(=O)Cc1ccc(C(C)(C)C)cc1[Se]c1ccccc1</smiles>

30 a

(CDCl $3,150 \mathrm{MHz}$ )

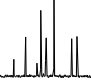

200
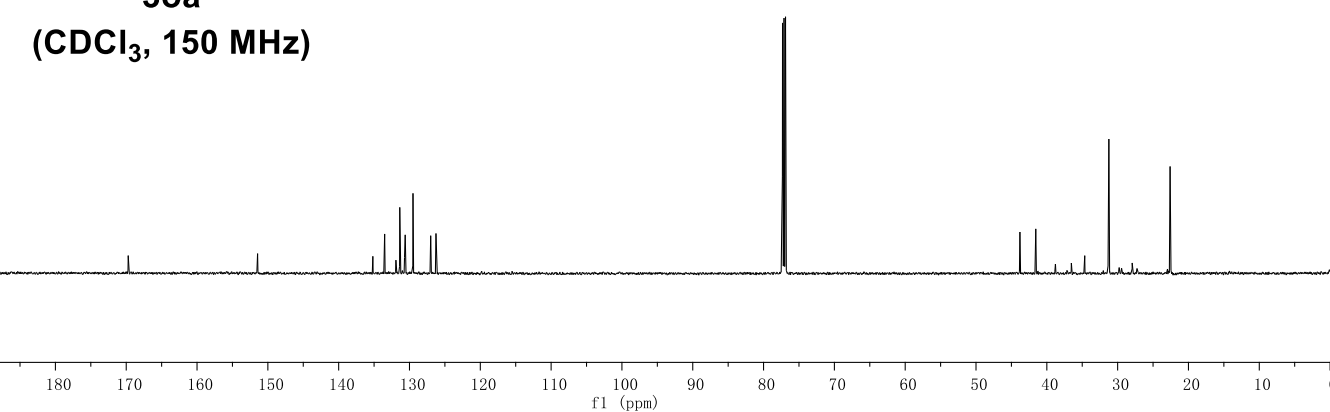
<smiles>CC(C)NC(=O)Cc1ccc(Br)cc1[Se]c1ccccc1</smiles>

3 pa

$\left(\mathrm{CDCl}_{3}, 600 \mathrm{MHz}\right)$

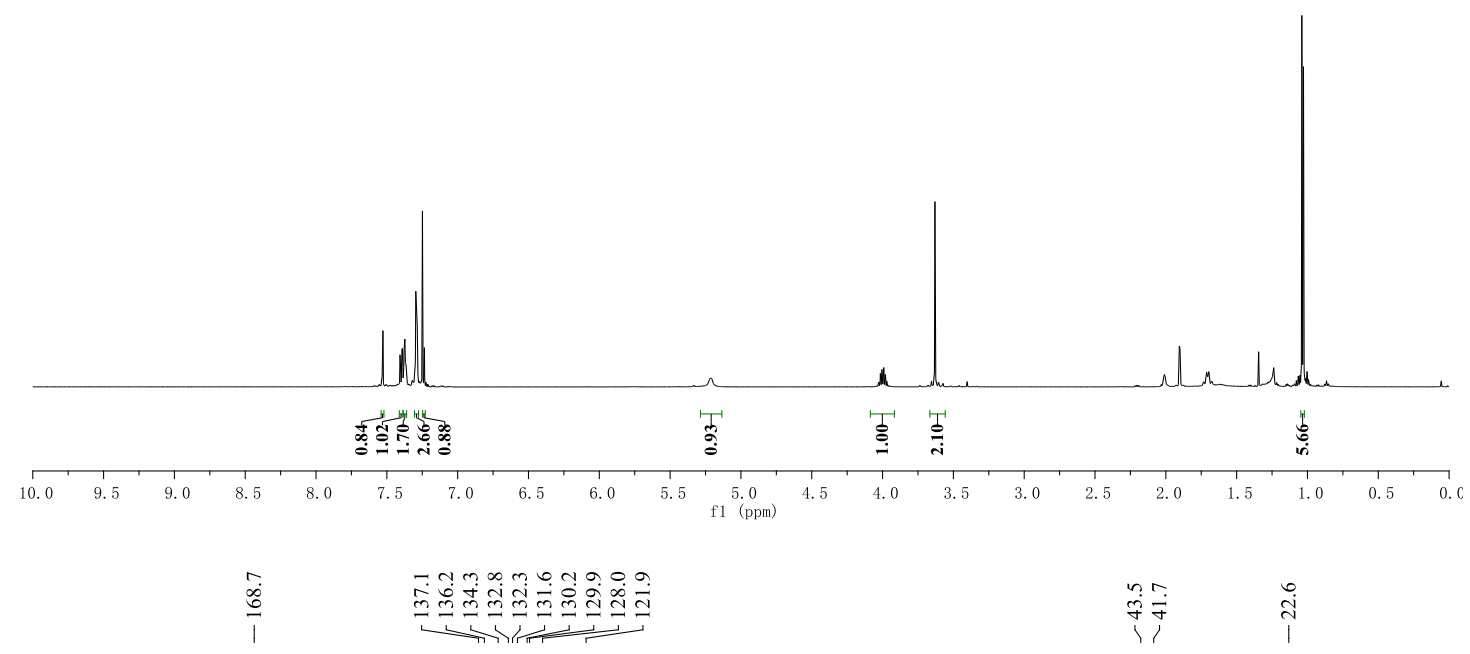<smiles>CC(C)NC(=O)Cc1ccc(Br)cc1[Se]c1ccccc1</smiles>

$3 p a$

(CDCl $3,150 \mathrm{MHz}$ )

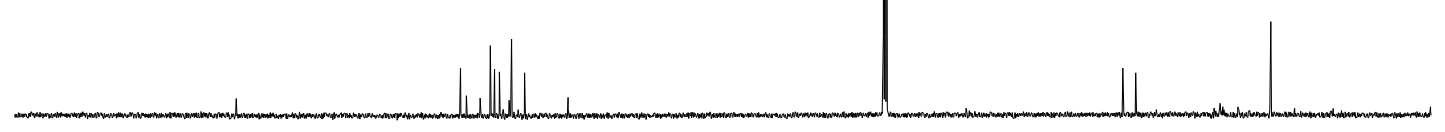

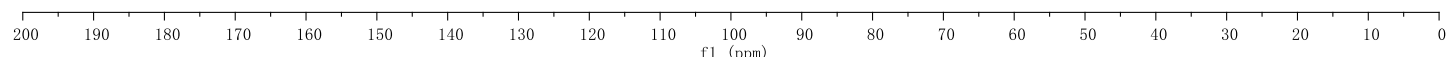




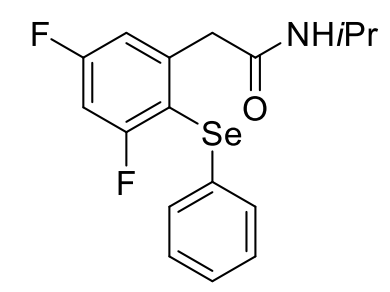

$3 q a$

$\left(\mathrm{CDCl}_{3}, 600 \mathrm{MHz}\right)$

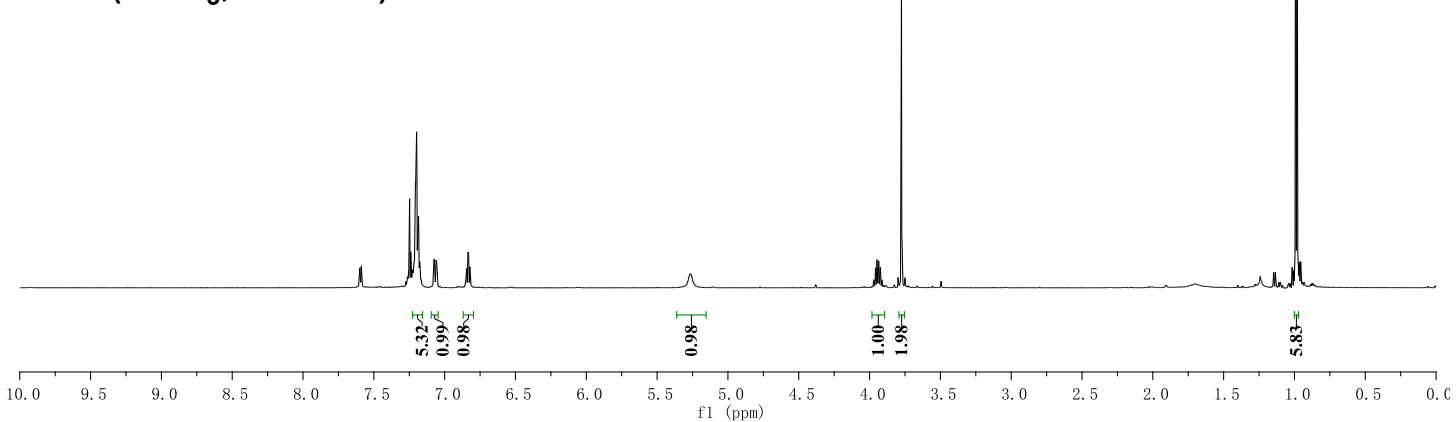

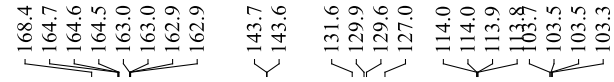<smiles>CC(C)NC(=O)Cc1cc(F)cc(F)c1[Se]c1ccccc1</smiles>

3qa

$\left(\mathrm{CDCl}_{3}, 150 \mathrm{MHz}\right)$

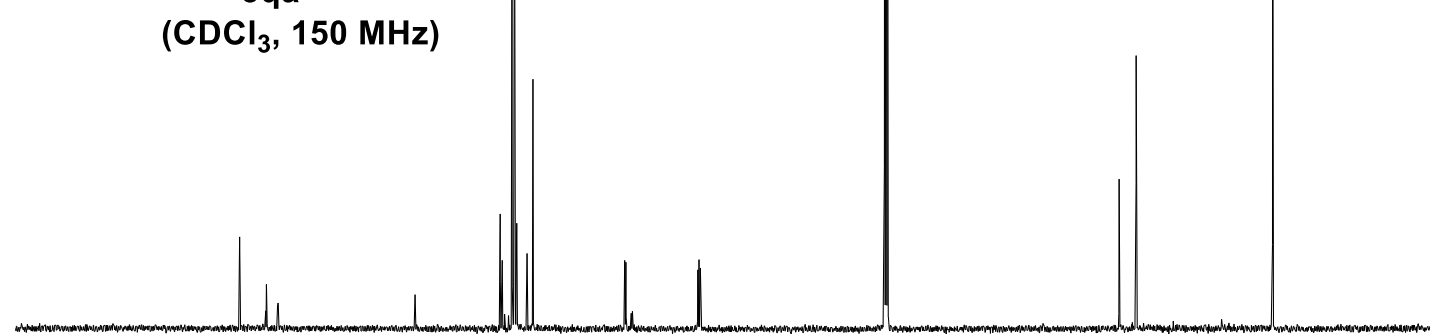

200

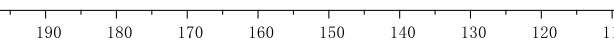

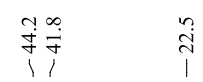

$\begin{array}{lllll}140 & 130 & 120 & 110 & 100 \\ \mathrm{f} 1(\mathrm{ppm}) & 90\end{array}$ 


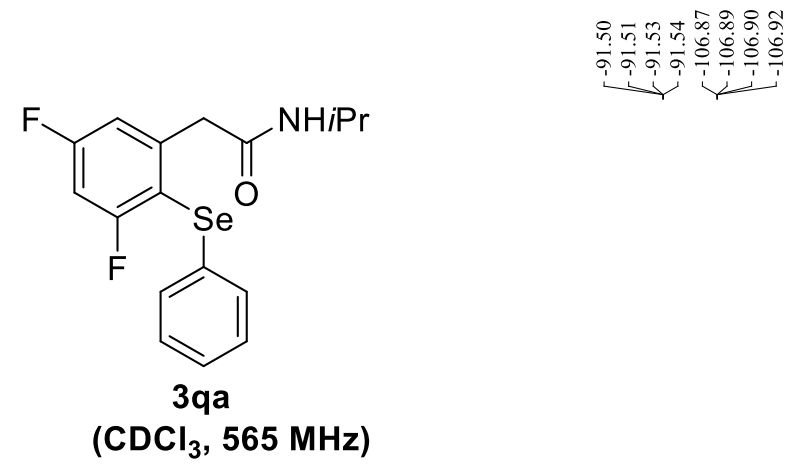

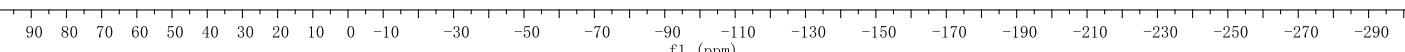



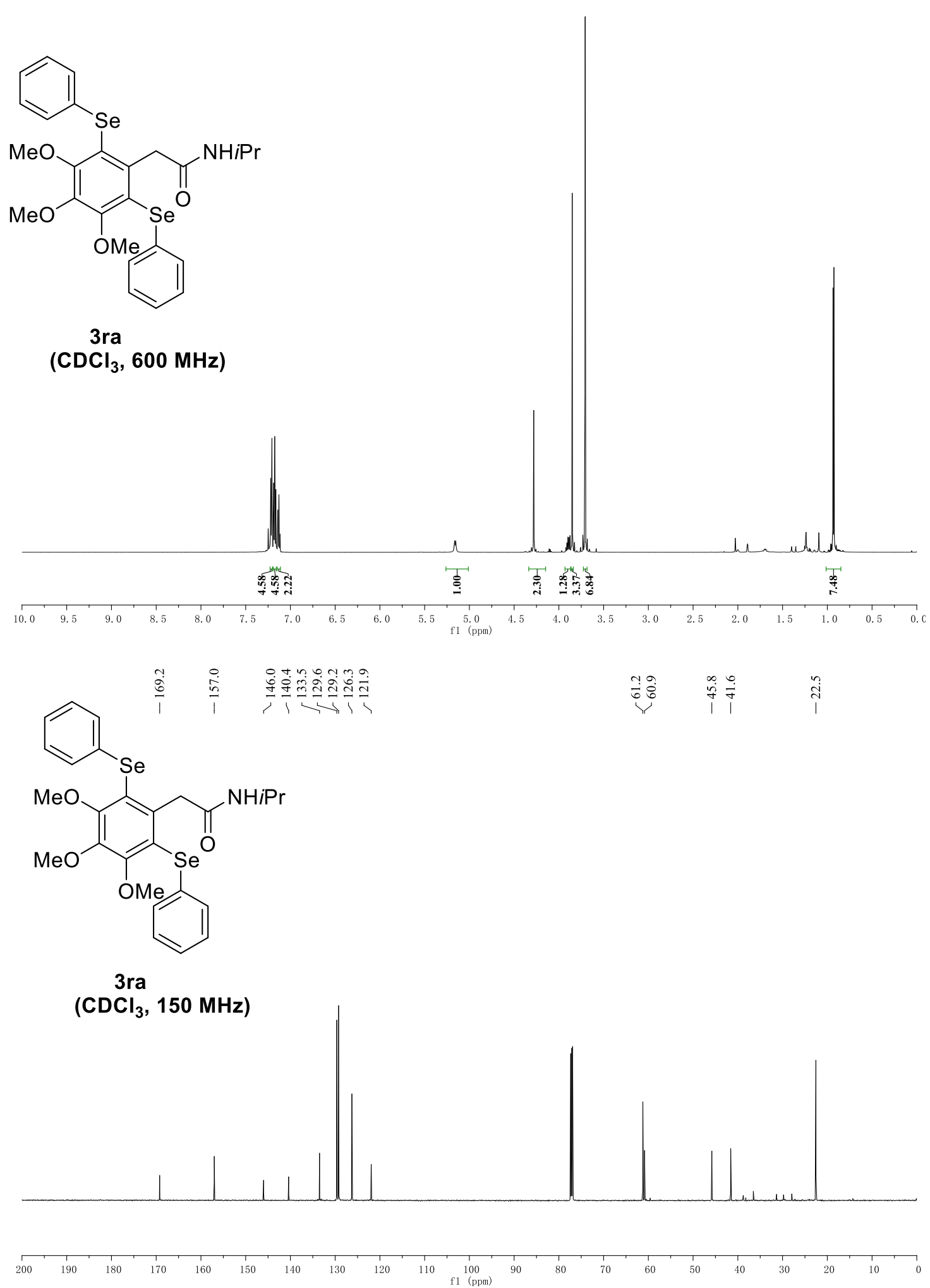
<smiles>Cc1ccc([Se]c2ccc(F)cc2)c(CC(=O)NC(C)C)c1</smiles>
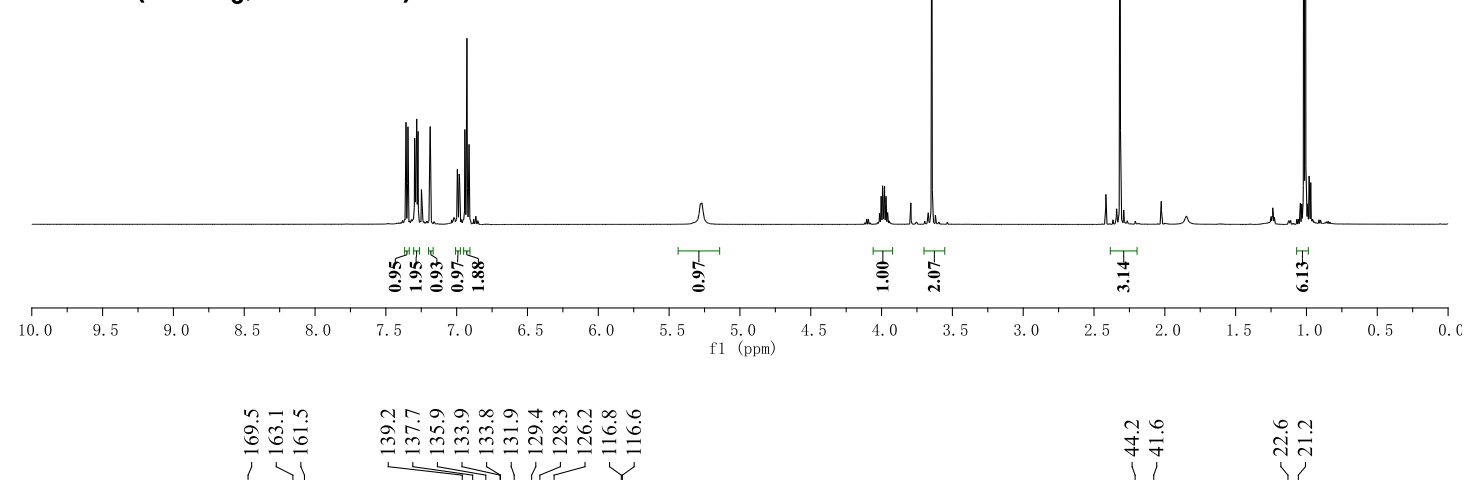<smiles>Cc1ccc([Se]c2ccc(F)cc2)c(CC(=O)NC(C)C)c1</smiles>

3ib

$\left(\mathrm{CDCl}_{3}, 150 \mathrm{MHz}\right)$

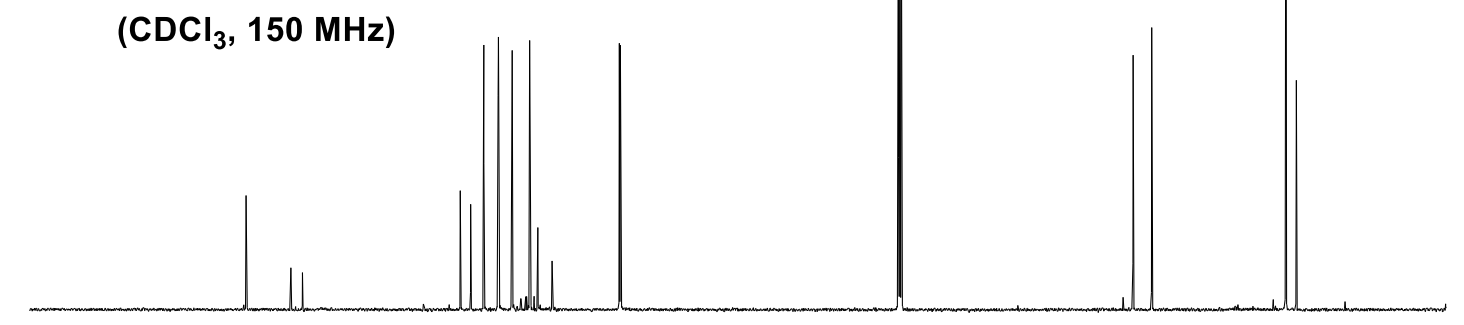

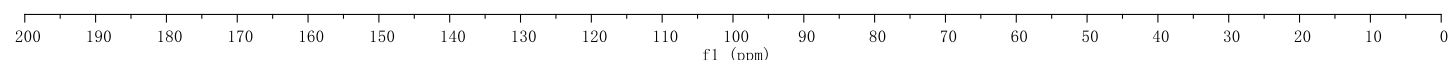




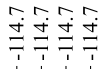

(NHiPr

$3 i b$

$\left(\mathrm{CDCl}_{3}, 565 \mathrm{MHz}\right)$

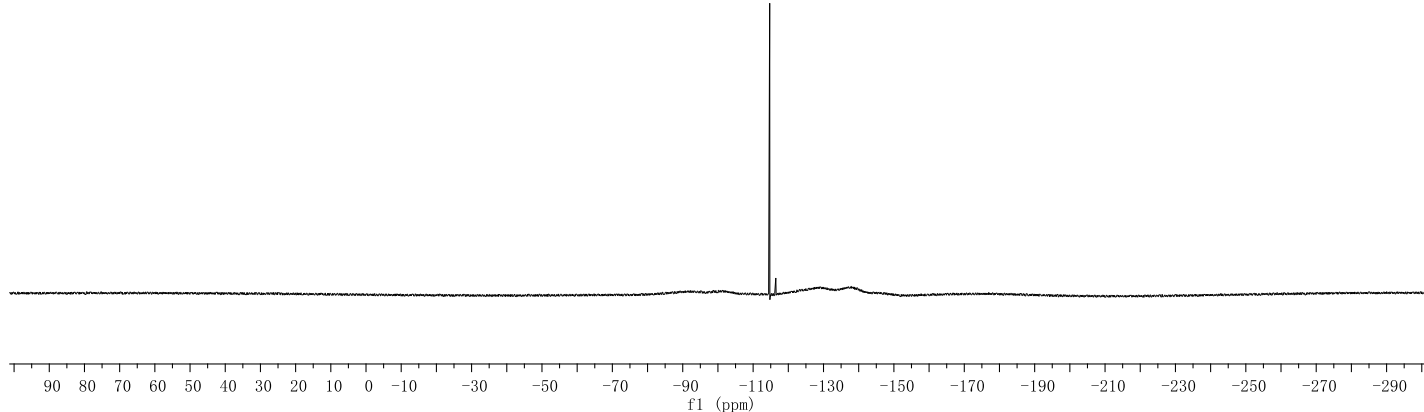




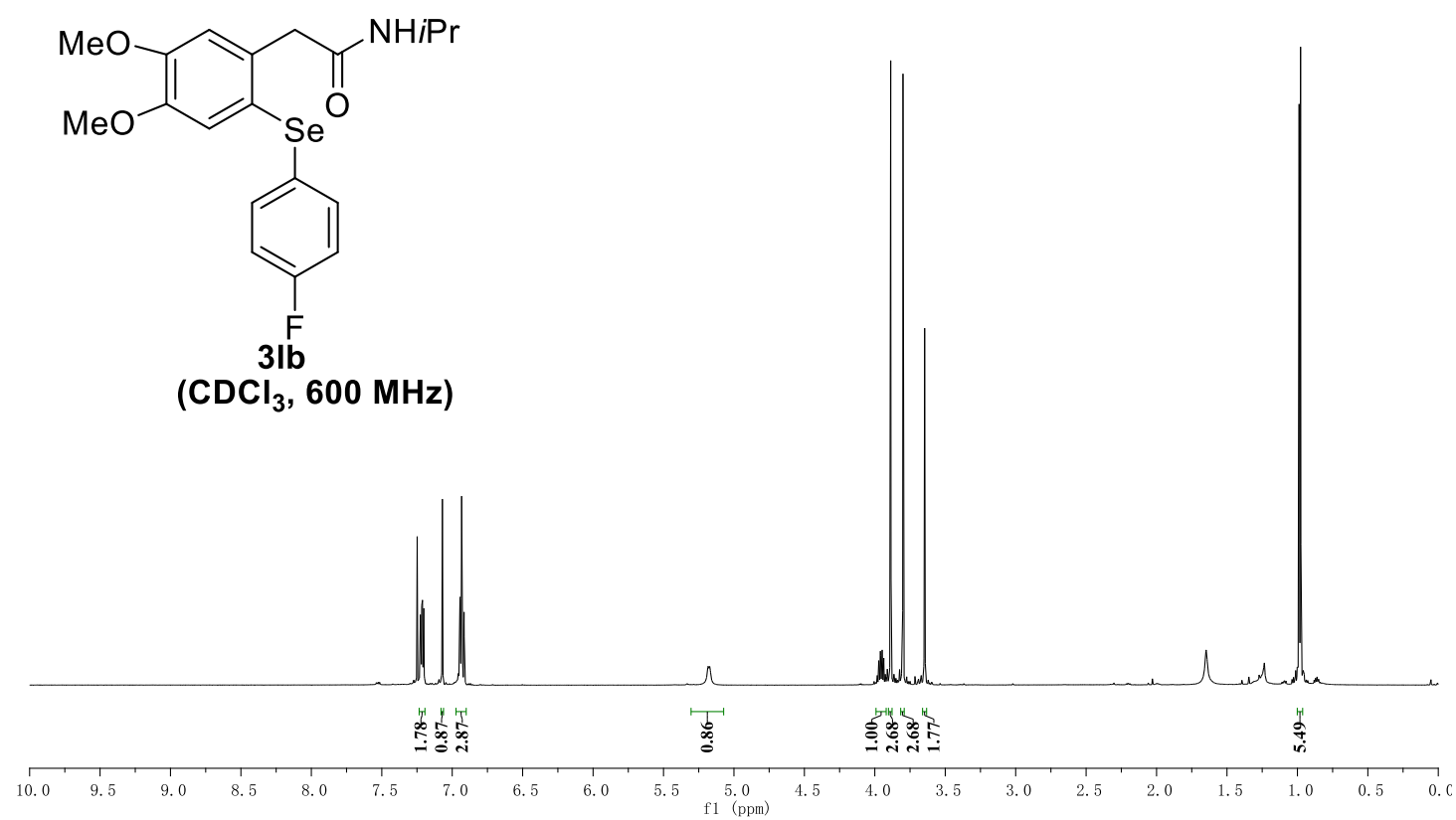

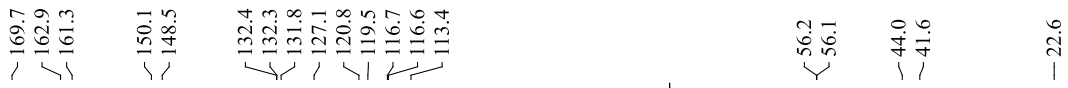<smiles>COc1cc([Se])c(CC(=O)NC(C)C)cc1OC</smiles>

$\left(\mathrm{CDCl}_{3}, 150 \mathrm{MHz}\right)$

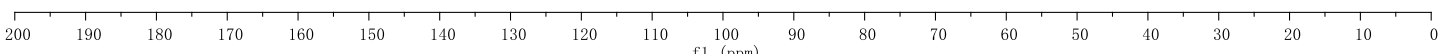




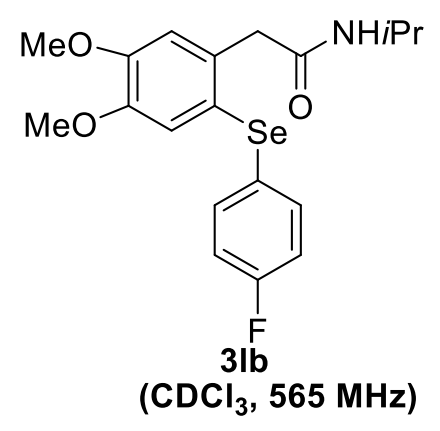

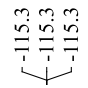

$\left(\mathrm{CDCl}_{3}, 565 \mathrm{MHz}\right.$ )

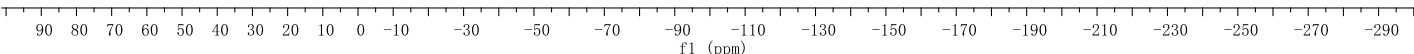



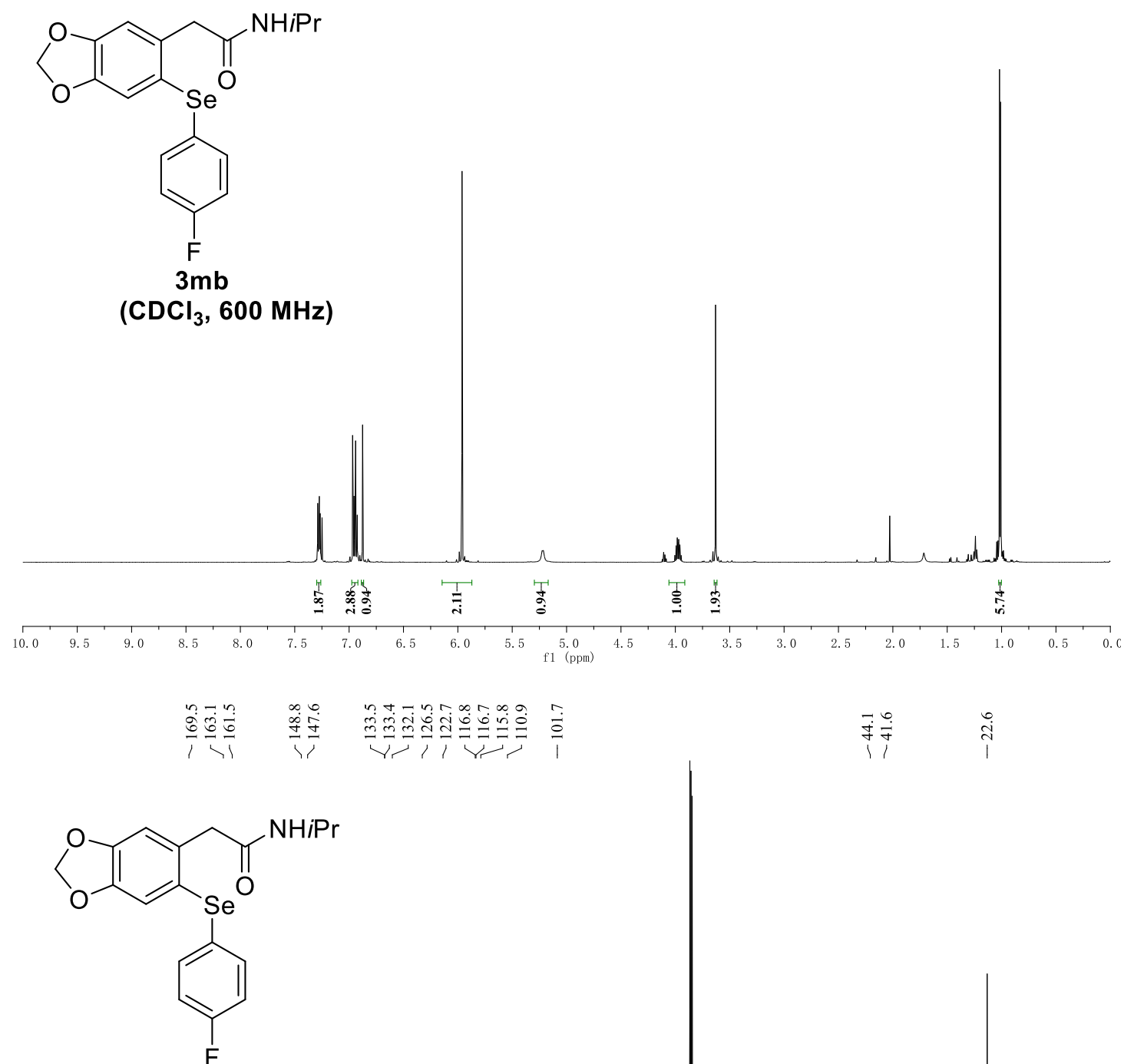

$3 \mathrm{mb}$

$\left(\mathrm{CDCl}_{3}, 150 \mathrm{MHz}\right)$

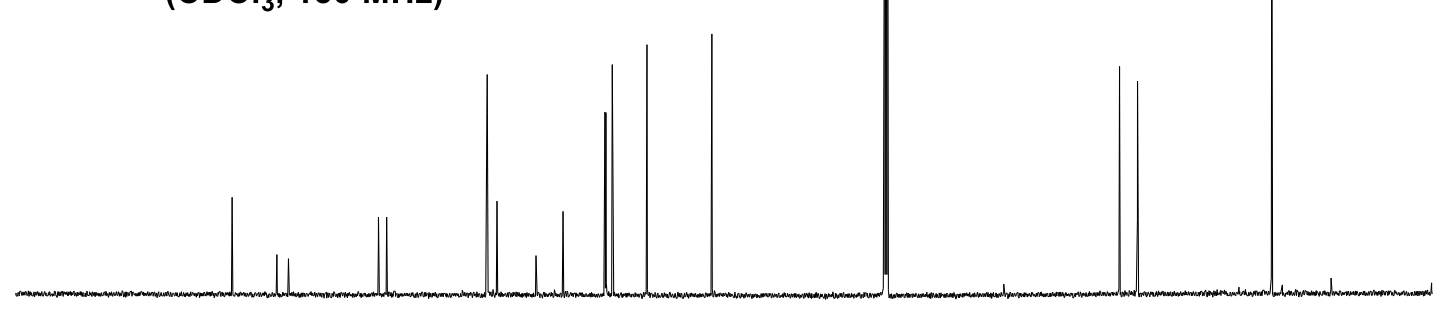

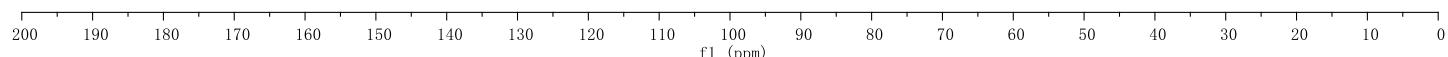




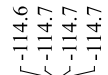

77

$y$<smiles>CC(C)NC(=O)Cc1cc2c(cc1[Se])OCO2</smiles>

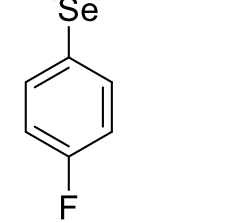

$3 \mathrm{mb}$

$\left(\mathrm{CDCl}_{3}, 565 \mathrm{MHz}\right)$

$\begin{array}{llllllllllllllllllllllllllll}90 & 80 & 70 & 60 & 50 & 40 & 30 & 20 & 10 & 0 & -10 & -30 & -50 & -70 & -90 & -110 & -130 & -150 & -170 & -190 & -210 & -230 & -250 & -270 & -290\end{array}$ 

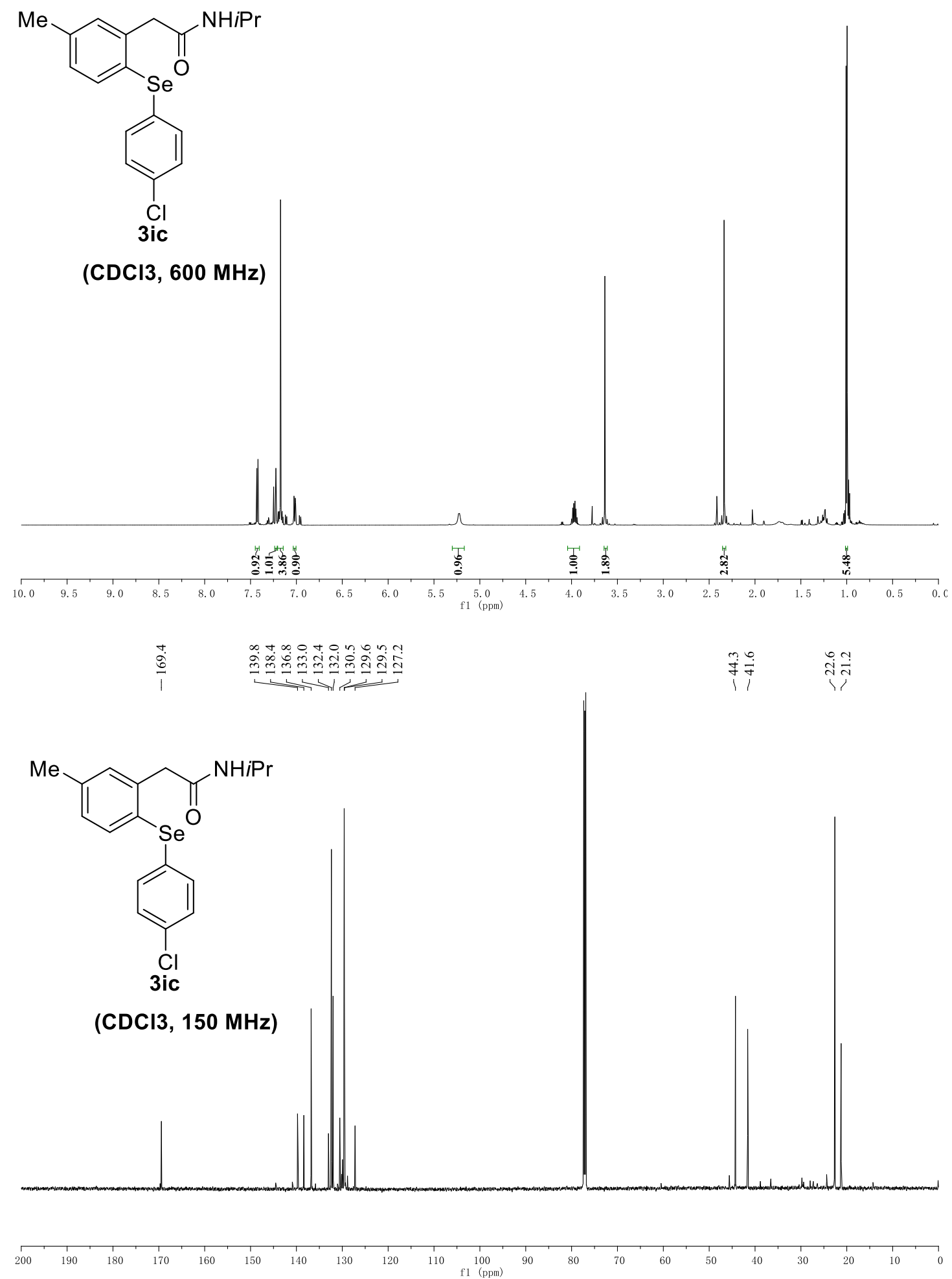
<smiles>Cc1ccc([Se]c2ccc(C(F)(F)F)cc2)c(CC(=O)NC(C)C)c1</smiles>

3id

(CDCl $3,600 \mathrm{MHz}$ )

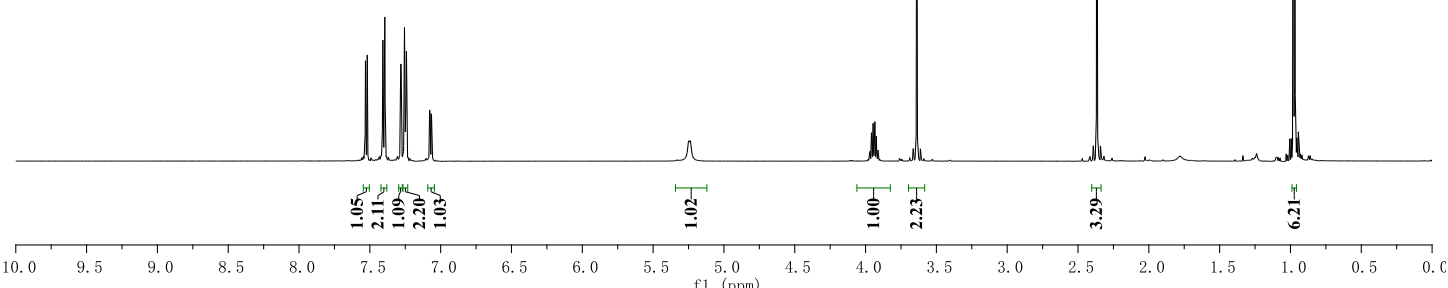

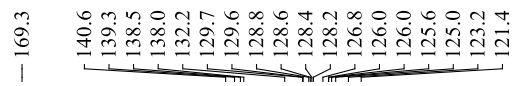<smiles>Cc1ccc([Se])c(CC(=O)NC(C)C)c1</smiles><smiles>Cc1ccc(C(F)(F)F)cc1</smiles>

3id

$\left(\mathrm{CDCl}_{3}, 150 \mathrm{MHz}\right)$

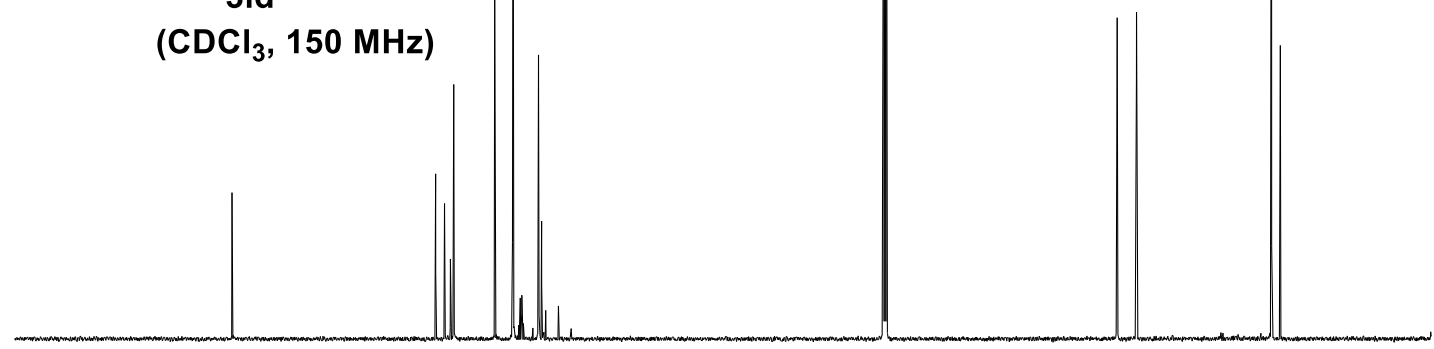

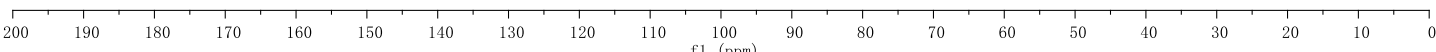


(CDCl, $565 \mathrm{MHz})$

$\begin{array}{llllllllllllllllllllllllllllll}90 & 80 & 70 & 60 & 50 & 40 & 30 & 20 & 10 & 0 & -10 & -30 & -50 & -70 & -90 & -110 & -130 & -150 & -170 & -190 & -210 & -230 & -250 & -270 & -290\end{array}$ 


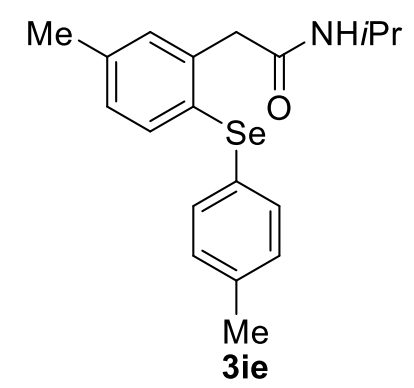

(CDCl $3,600 \mathrm{MHz}$ )
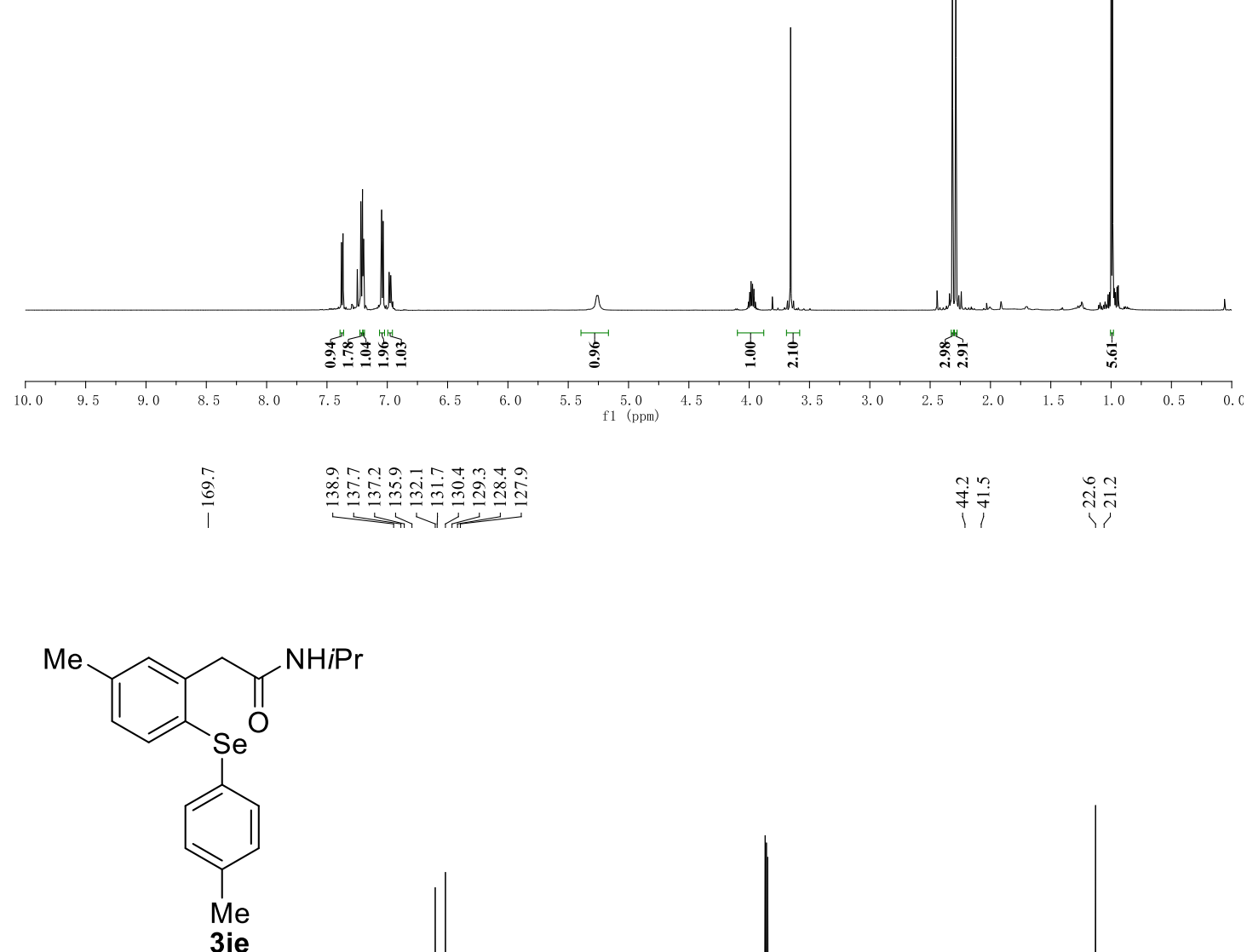

$\left(\mathrm{CDCl}_{3}, 150 \mathrm{MHz}\right)$

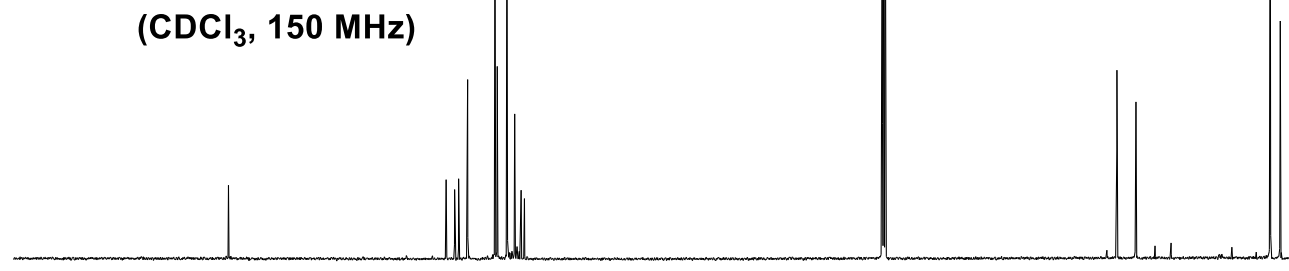

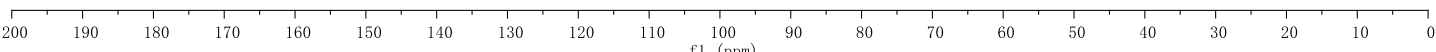



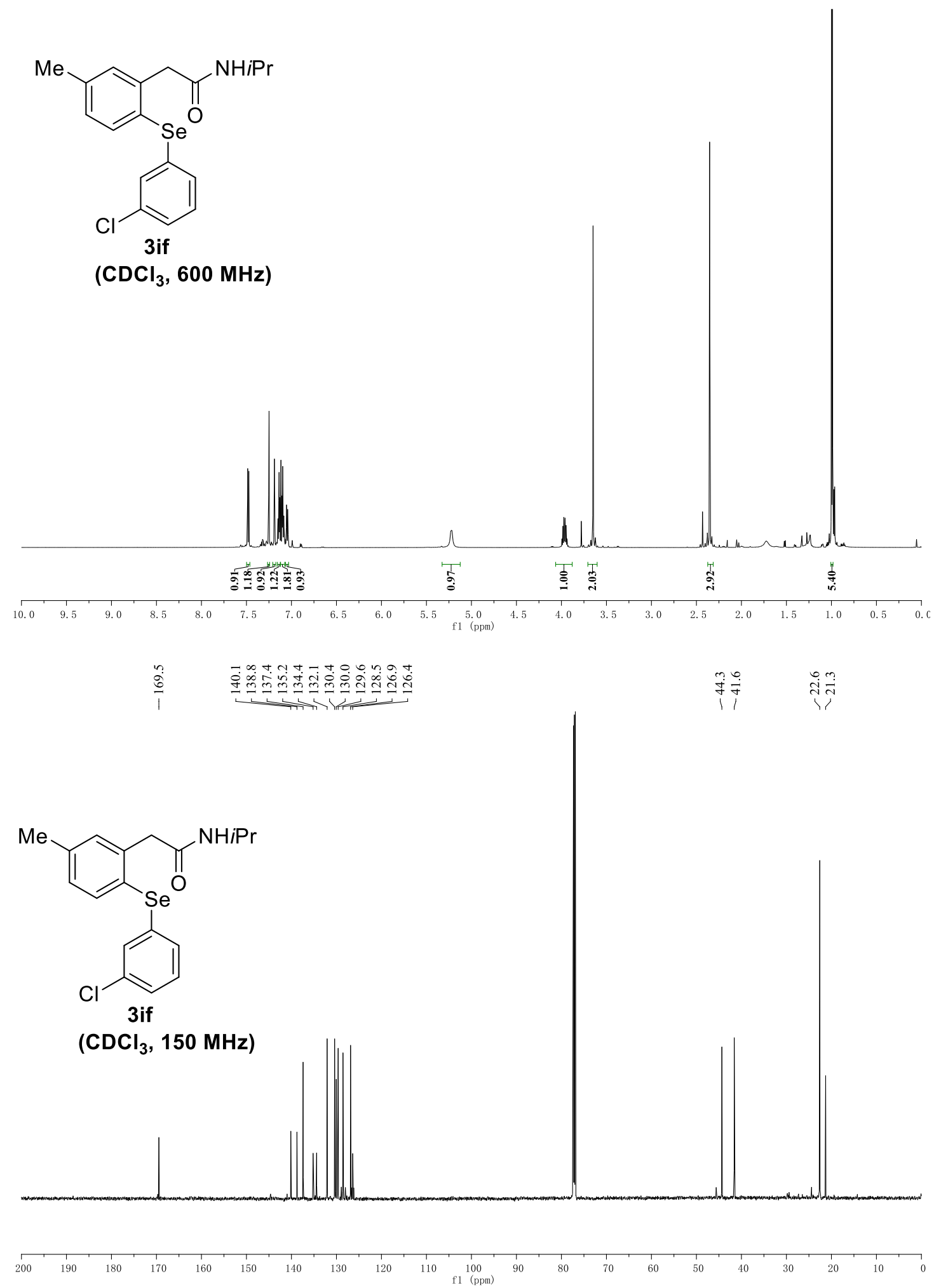

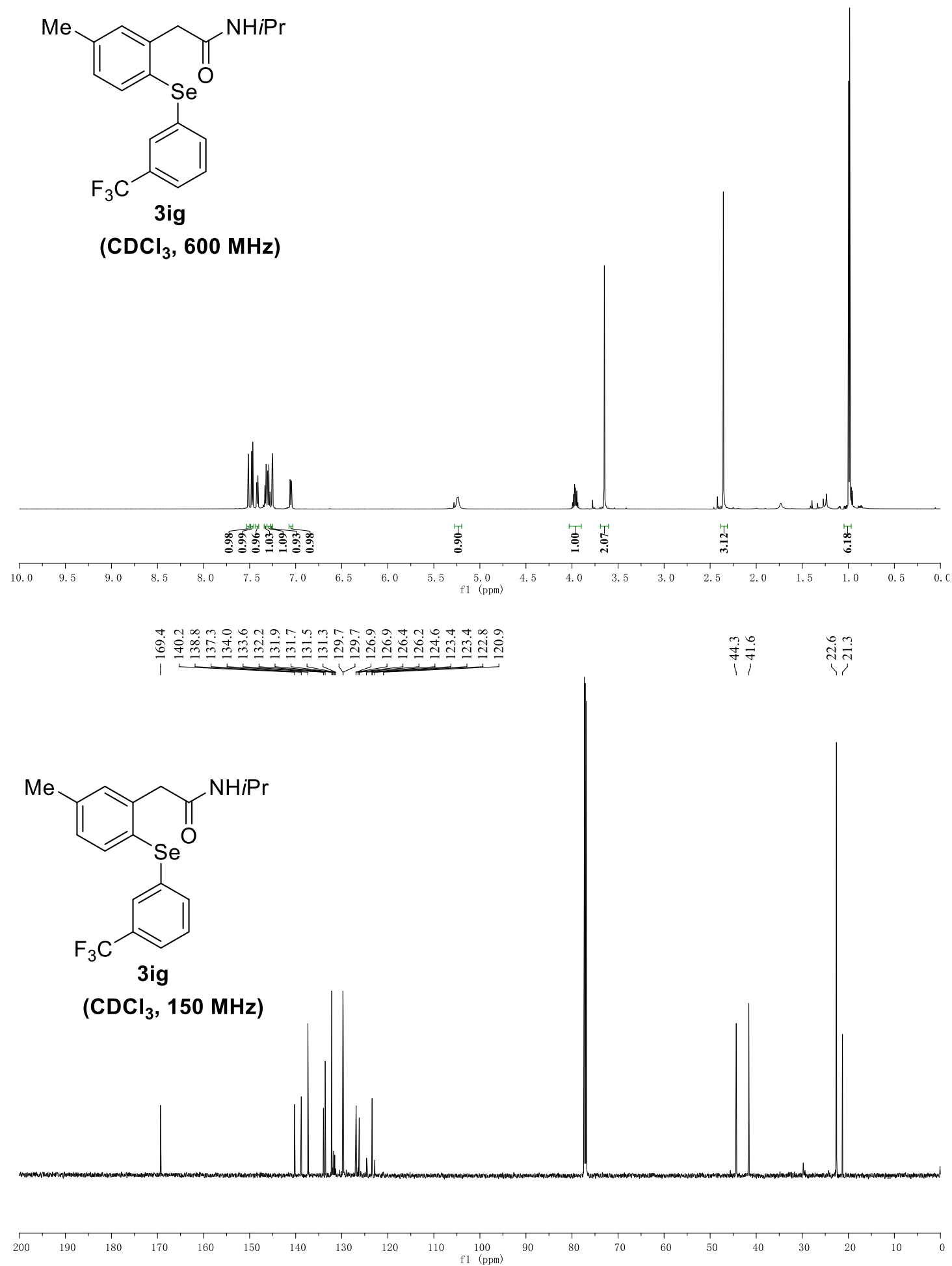


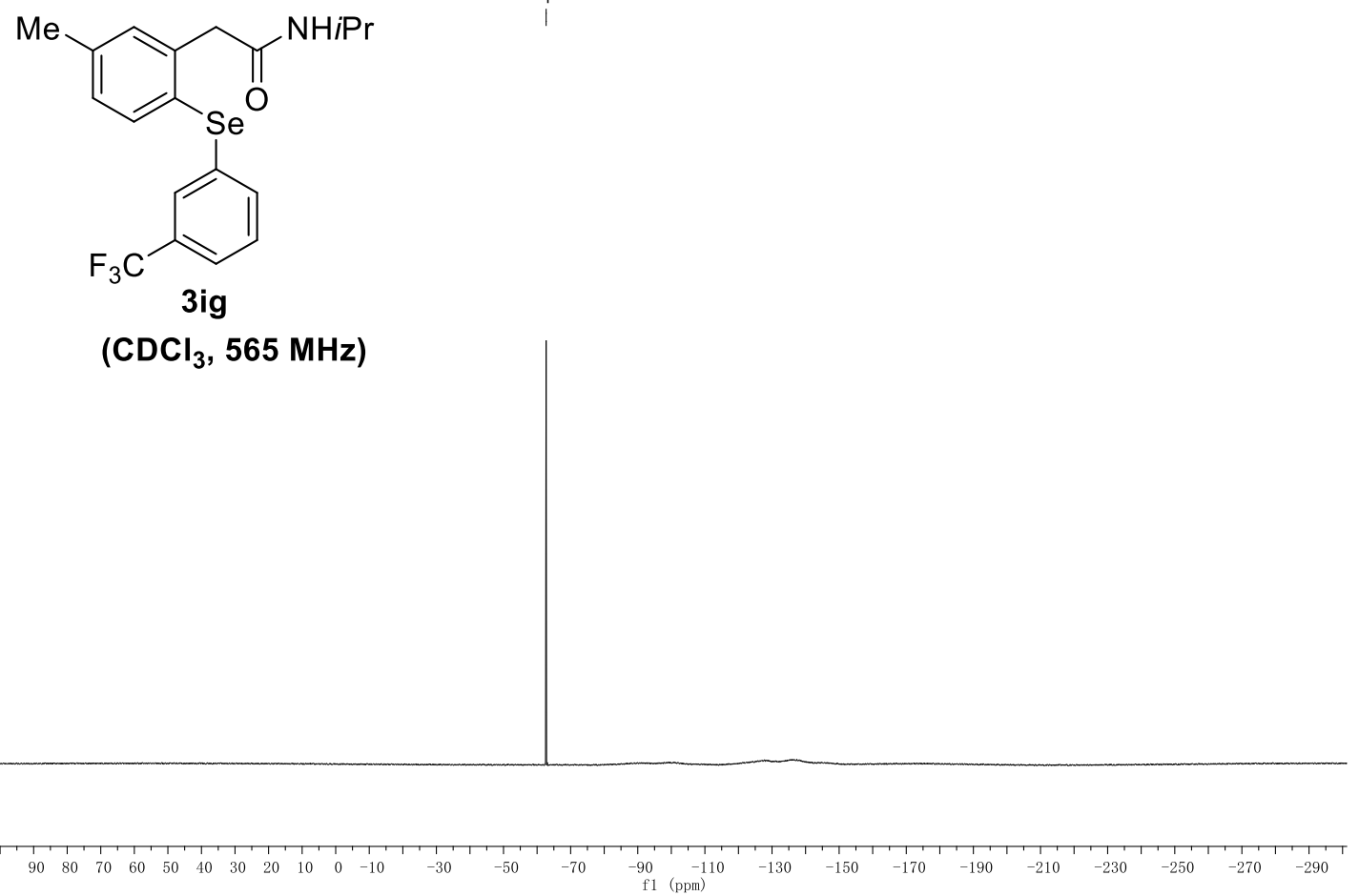


<smiles>Cc1ccc([Se]c2ccccc2F)c(CC(=O)NC(C)C)c1</smiles>

3 ih

$\left(\mathrm{CDCl}_{3}, 600 \mathrm{MHz}\right)$

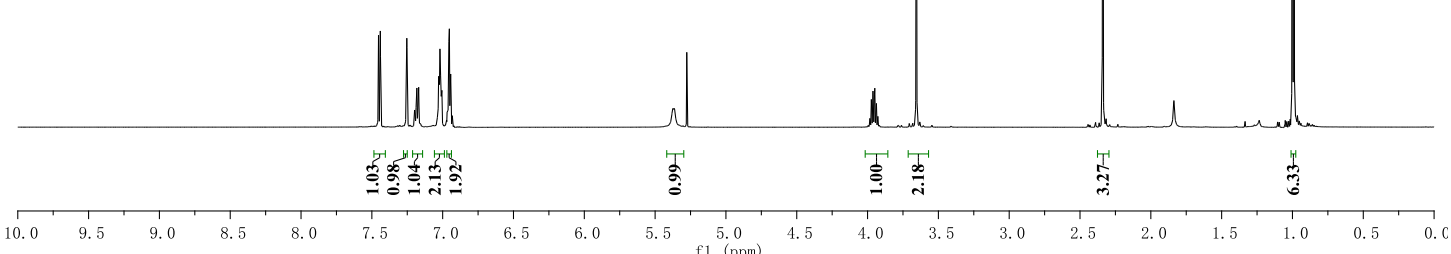

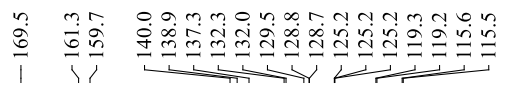

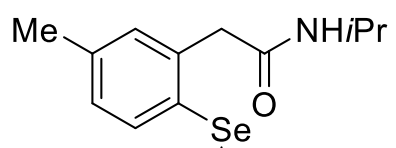<smiles>Cc1ccccc1F</smiles>

3ih

$\left(\mathrm{CDCl}_{3}, 150 \mathrm{MHz}\right)$

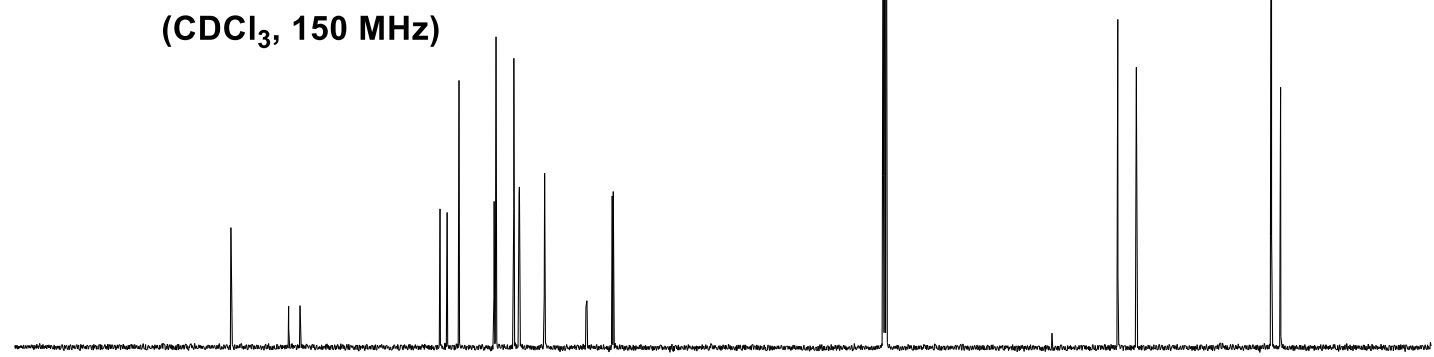

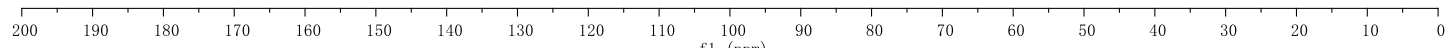


(NHiPr

3 ih

$\left(\mathrm{CDCl}_{3}, 565 \mathrm{MHz}\right)$

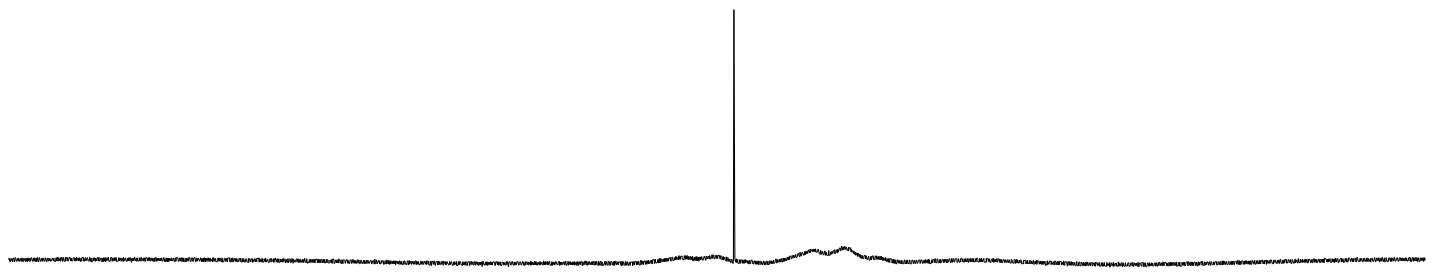

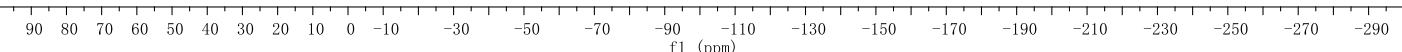




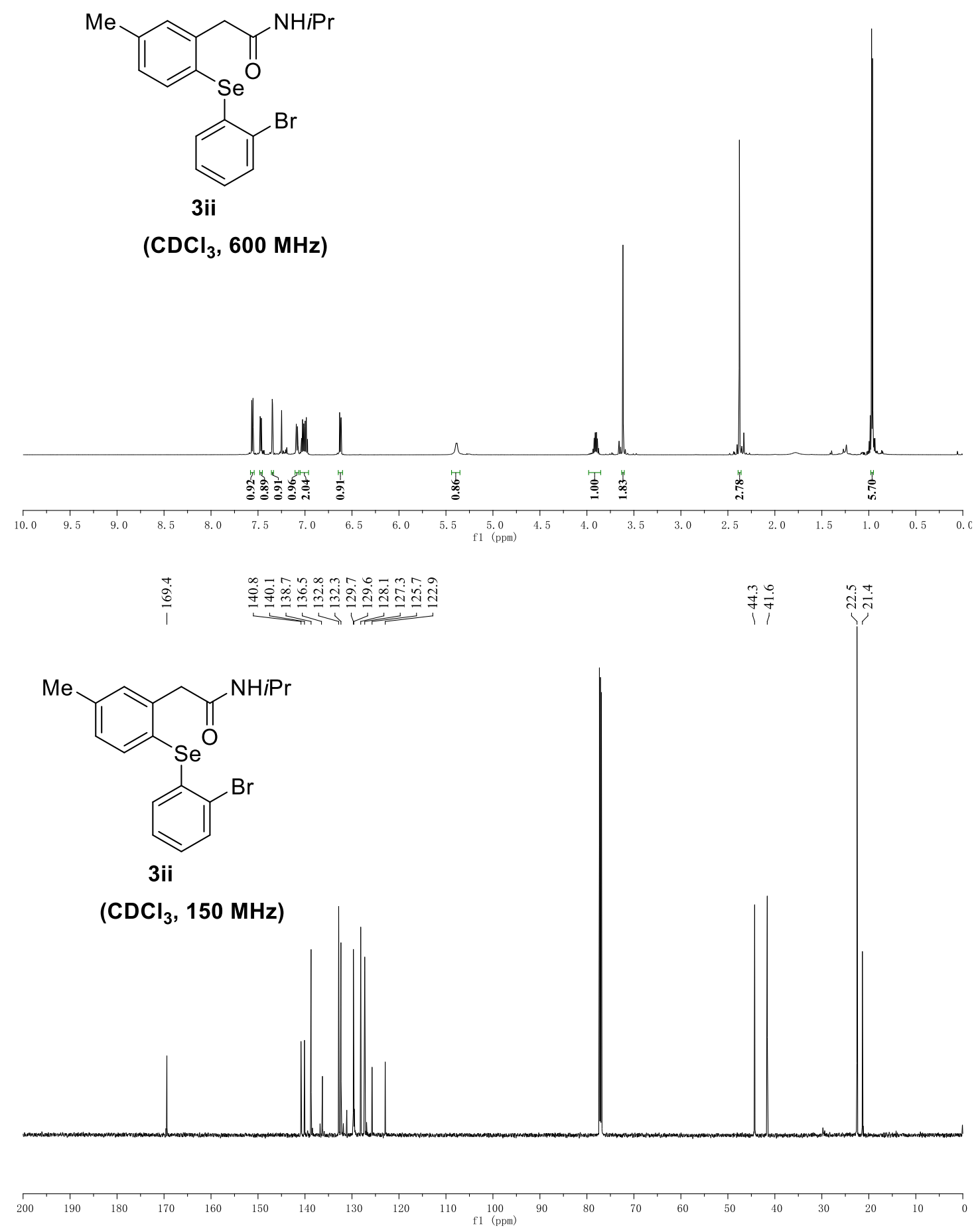




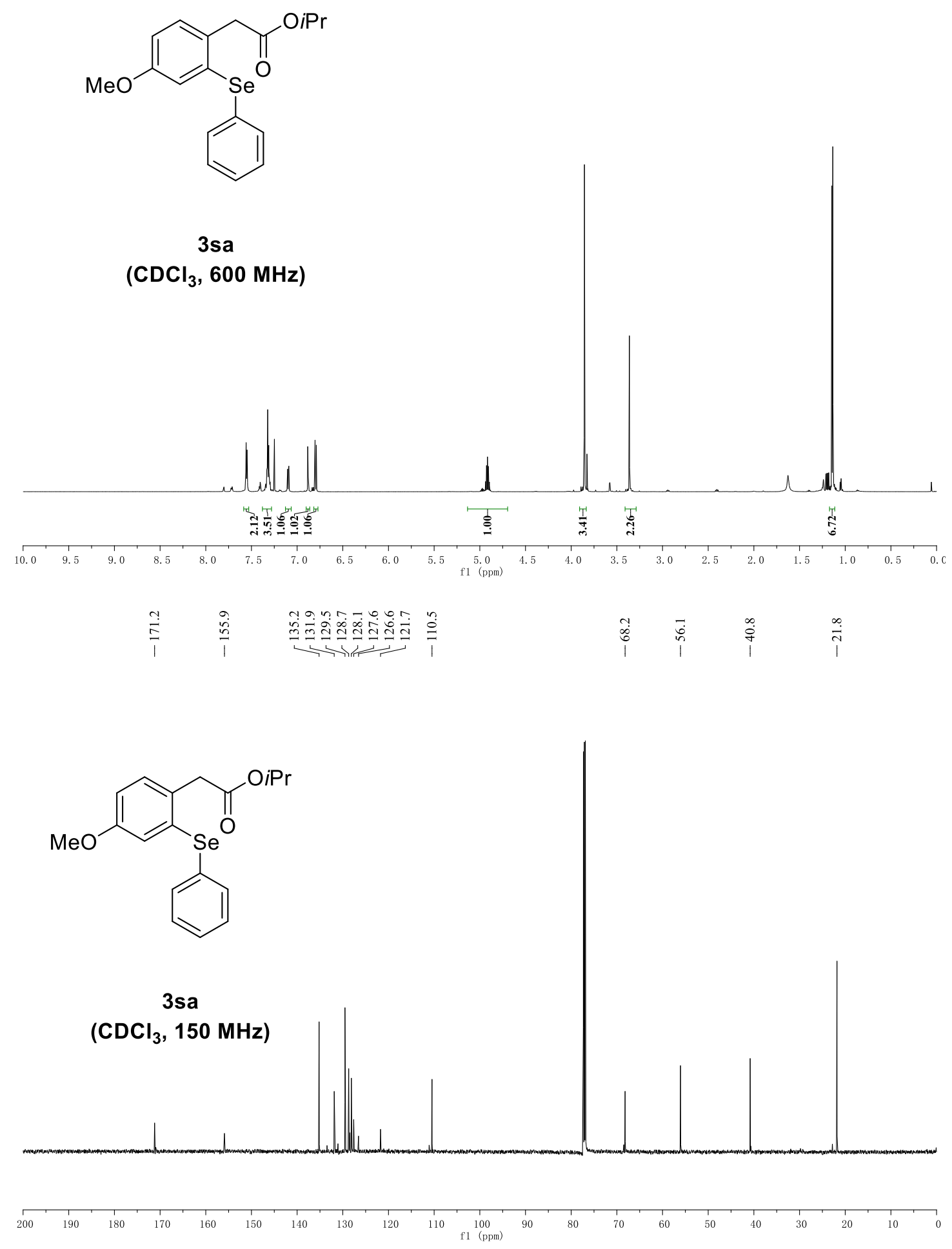




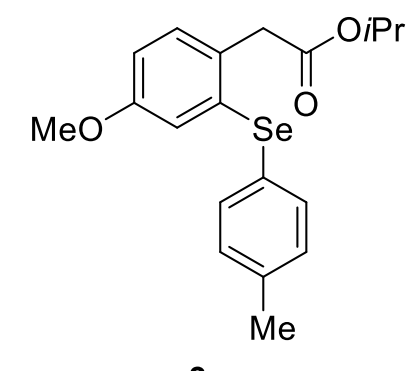

( $\mathrm{CDCl}_{3}, 600 \mathrm{MHz}$ )

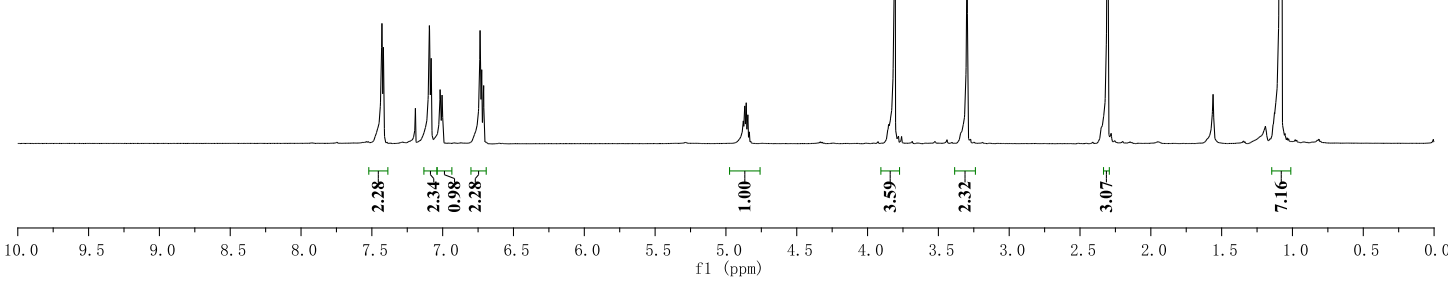

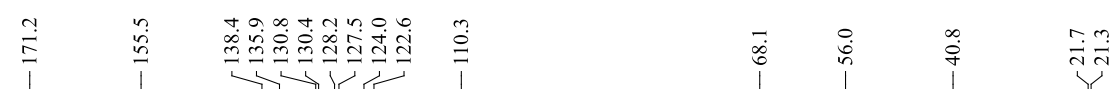

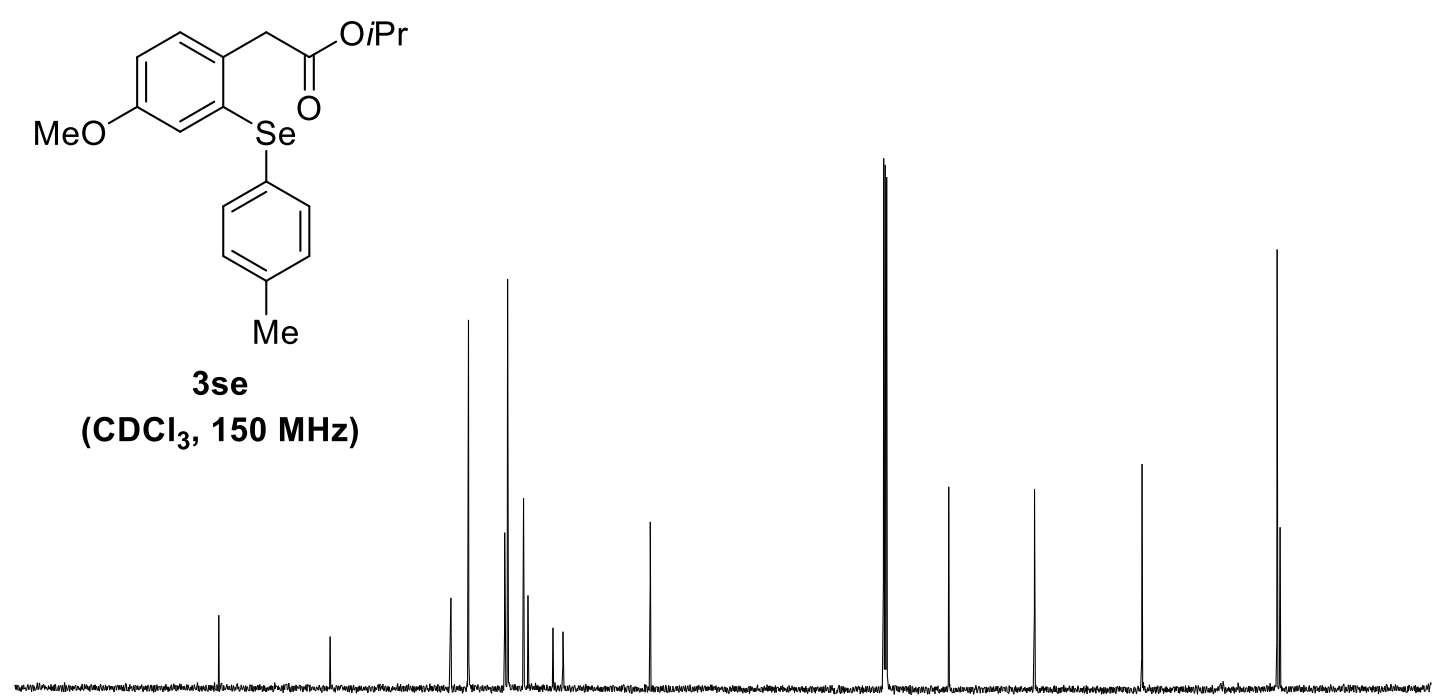

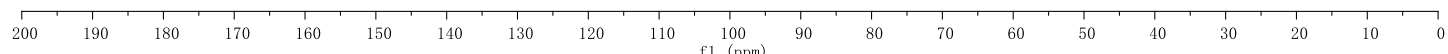


<smiles>CCCOC(=O)Cc1ccc(OC)cc1[Se]c1ccc(Br)cc1</smiles>

3sj

$\left(\mathrm{CDCl}_{3}, 600 \mathrm{MHz}\right)$

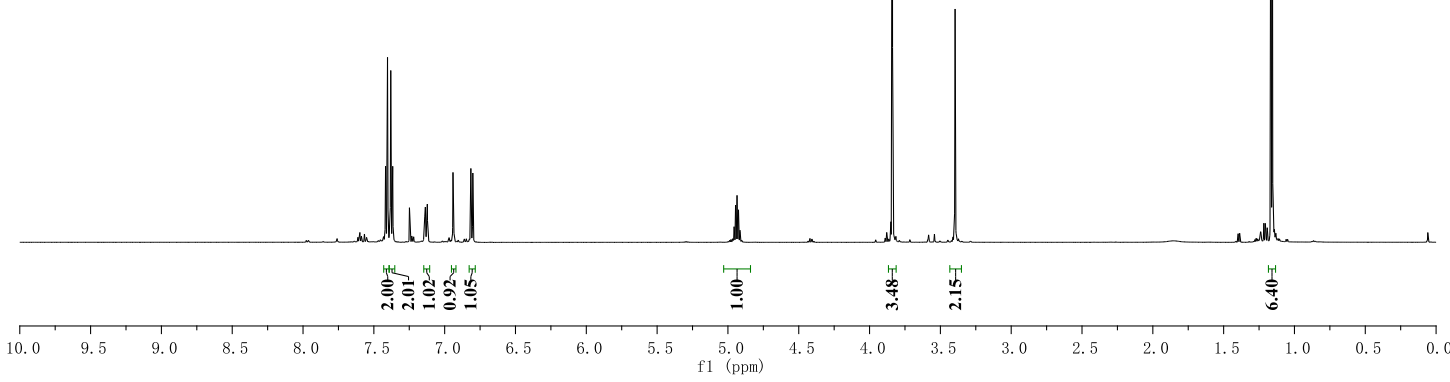

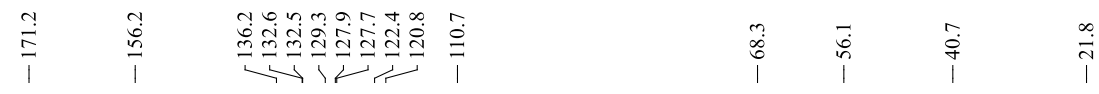<smiles>COc1ccc(CC(=O)OC(C)C)c([Se]c2ccc(Br)cc2)c1</smiles>

3sj

$\left(\mathrm{CDCl}_{3}, 150 \mathrm{MHz}\right)$

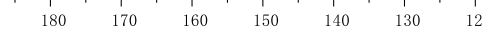

$10 \quad \begin{aligned} & 100 \\ & \mathrm{f} 1\end{aligned}$ 

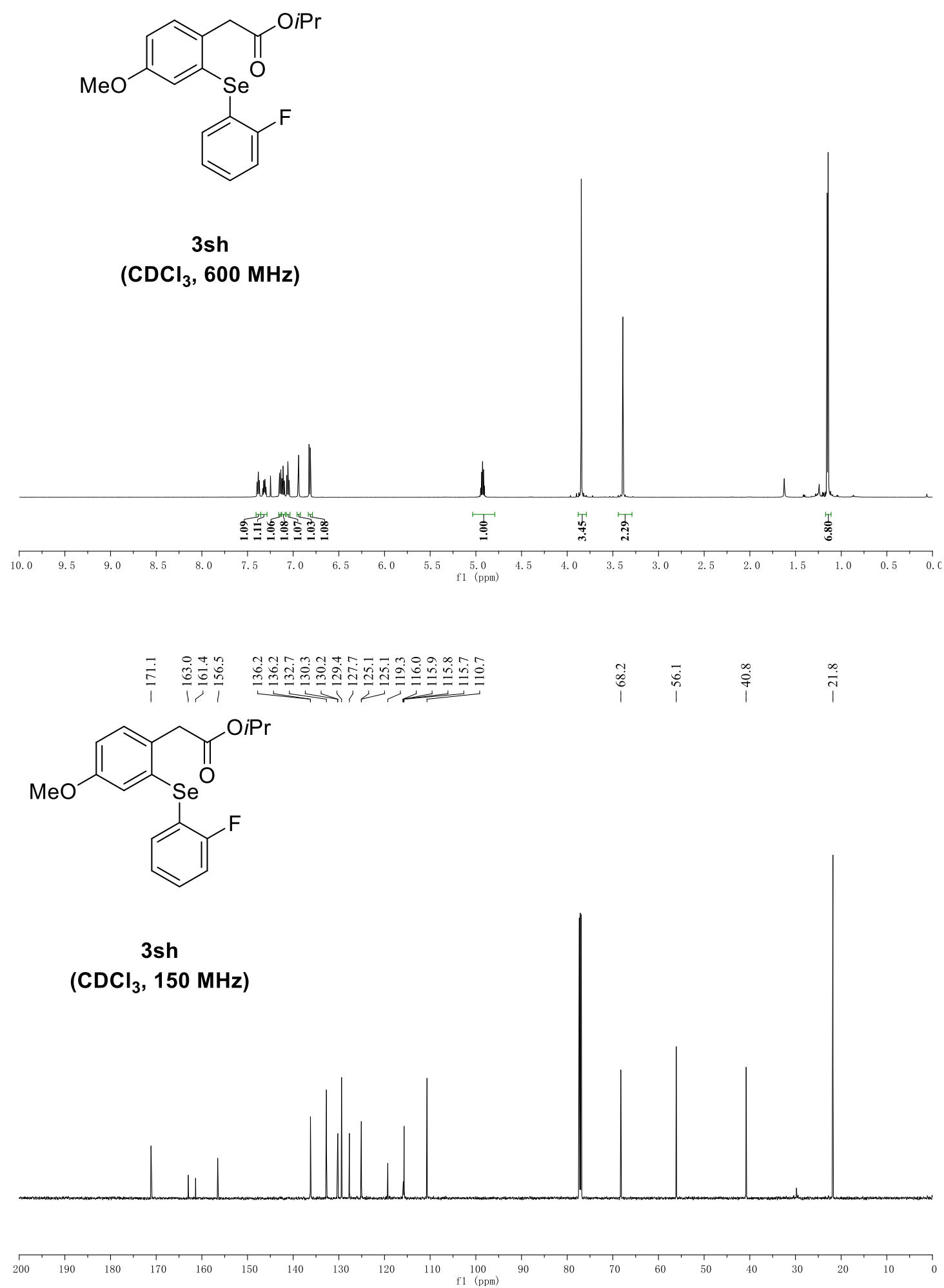
<smiles>CCOC(=O)Cc1ccc(OC)cc1OC(C)C</smiles>

3sh

$\left(\mathrm{CDCl}_{3}, 565 \mathrm{MHz}\right)$

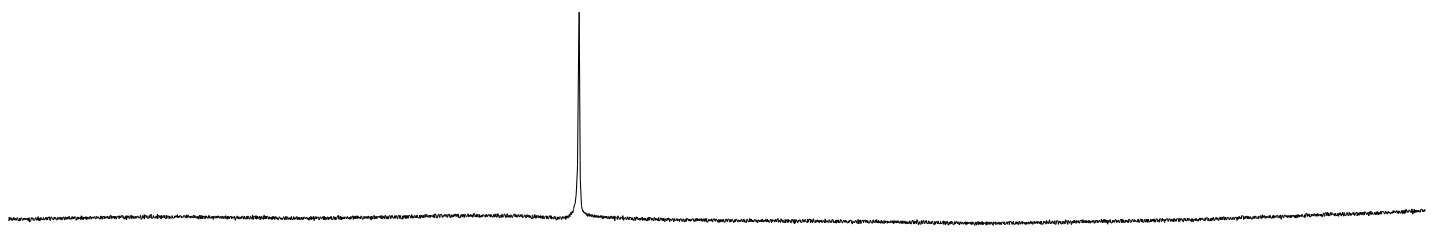

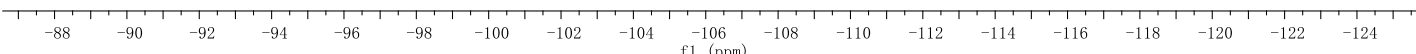




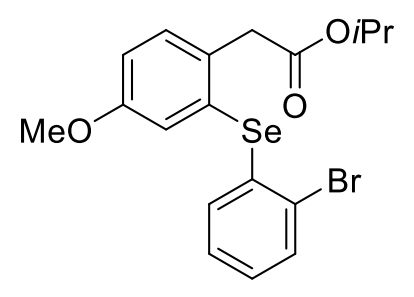

3 si

$\left(\mathrm{CDCl}_{3}, 600 \mathrm{MHz}\right)$

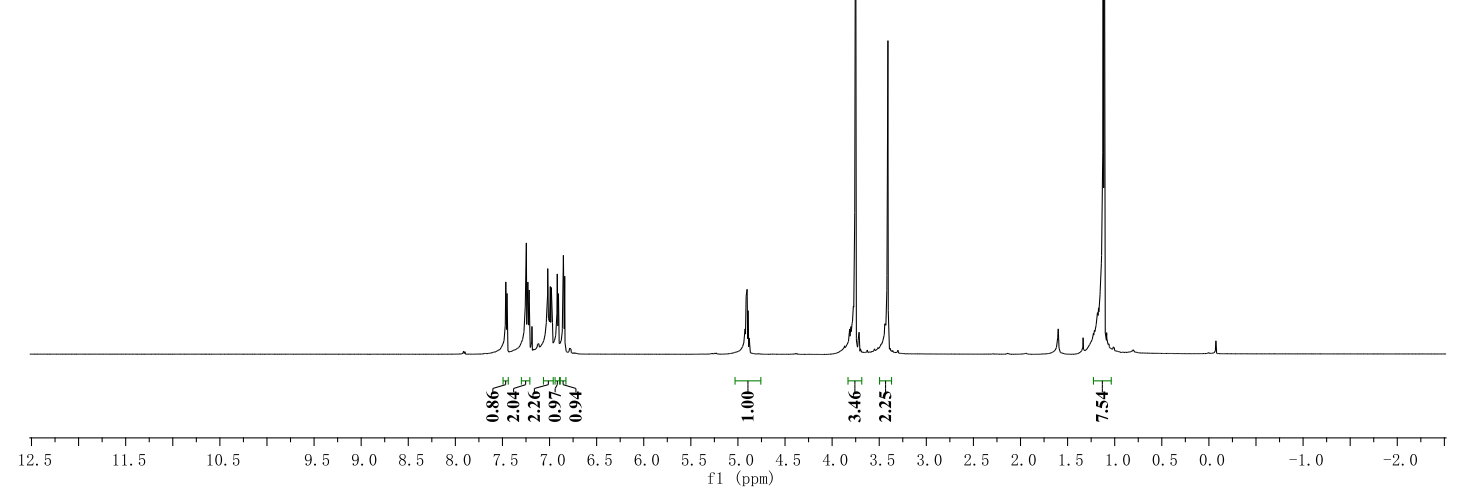

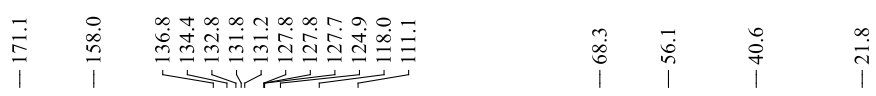<smiles>CCCOC(=O)Cc1ccc(OC)cc1[Se]c1ccccc1Br</smiles>

$3 s \mathrm{i}$

$\left(\mathrm{CDCl}_{3}, 150 \mathrm{MHz}\right)$

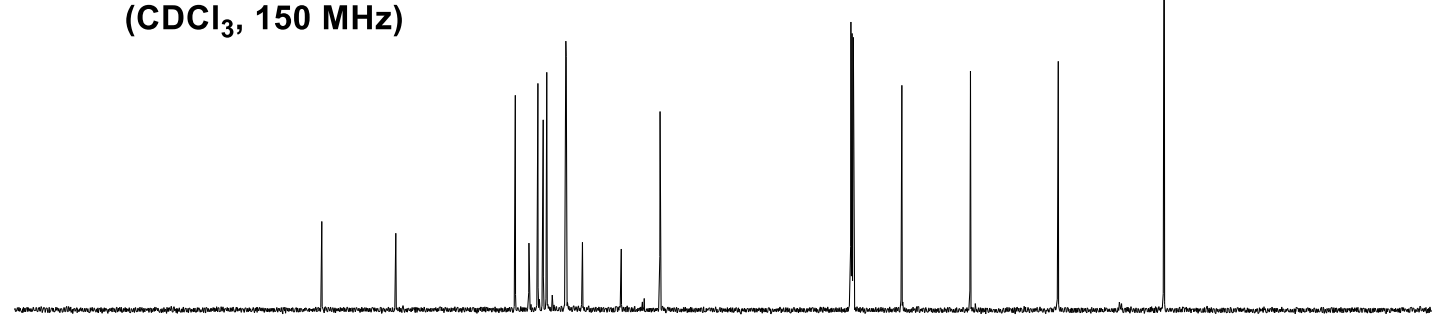

$\begin{array}{lllllllllllllllllllllllllllll}1 & 1 \\ 220 & 210 & 200 & 190 & 180 & 170 & 160 & 150 & 140 & 130 & 120 & 110 & 100 & 90 & 80 & 70 & 60 & 50 & 40 & 30 & 20 & 10 & 0 & -10 & -20\end{array}$ 


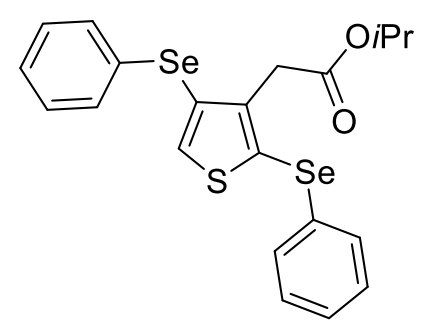

3ta

$\left(\mathrm{CDCl}_{3}, 600 \mathrm{MHz}\right)$

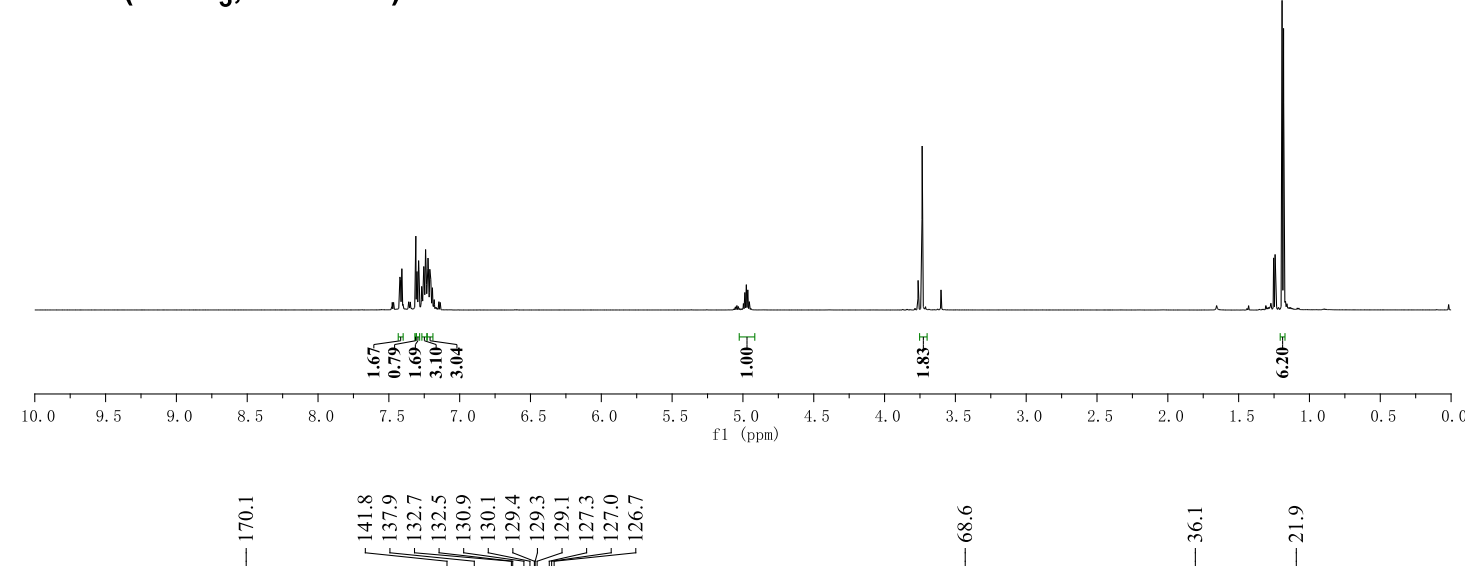<smiles>CC(C)OC(=O)Cc1c([Se]c2ccccc2)csc1[Se]c1ccccc1</smiles>

3 ta

$\left(\mathrm{CDCl}_{3}, 150 \mathrm{MHz}\right)$

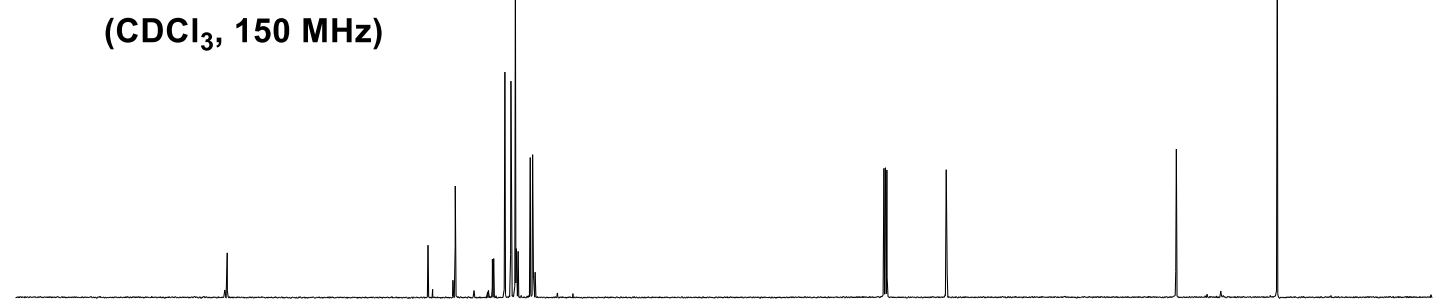

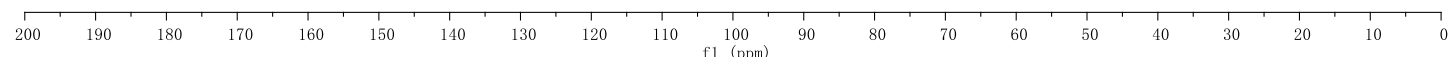


<smiles>CCCOC(=O)Cc1ccc(OC)cc1Sc1ccccc1</smiles>

3sk

$\left(\mathrm{CDCl}_{3}, 600 \mathrm{MHz}\right)$

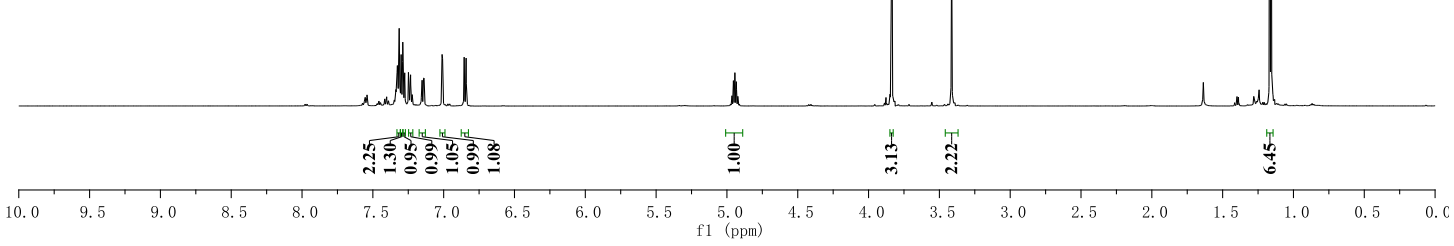

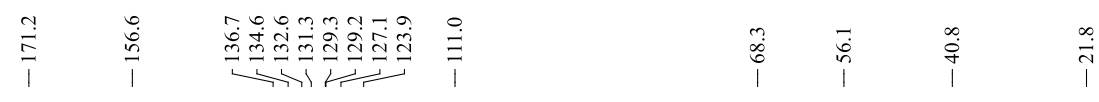<smiles>CCCOC(=O)Cc1ccc(OC)cc1Sc1ccccc1</smiles>

3sk

$\left(\mathrm{CDCl}_{3}, 150 \mathrm{MHz}\right)$

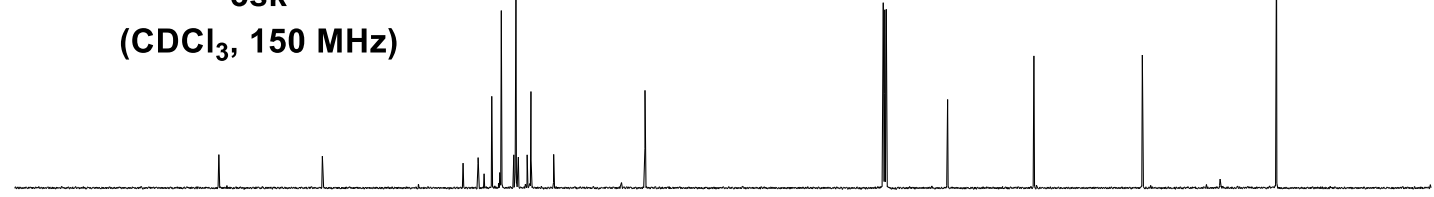

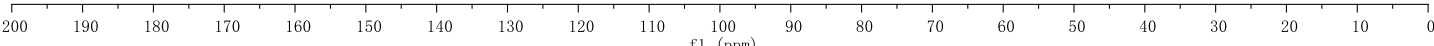




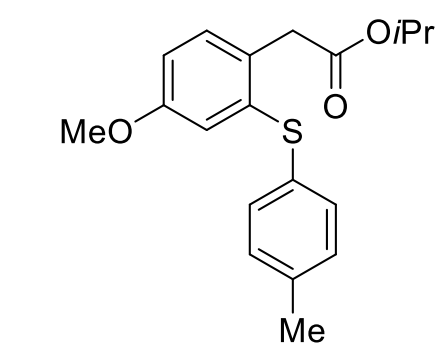

$3 s 1$

$\left(\mathrm{CDCl}_{3}, 600 \mathrm{MHz}\right)$

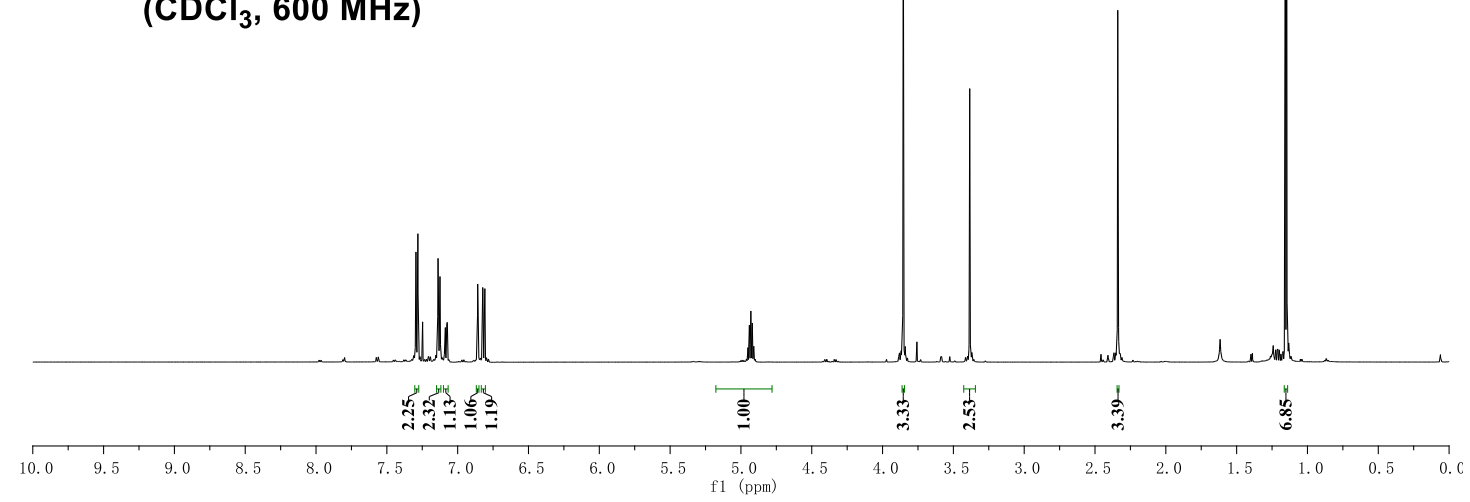

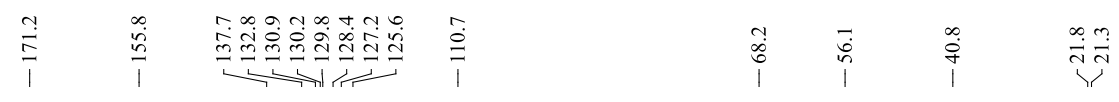

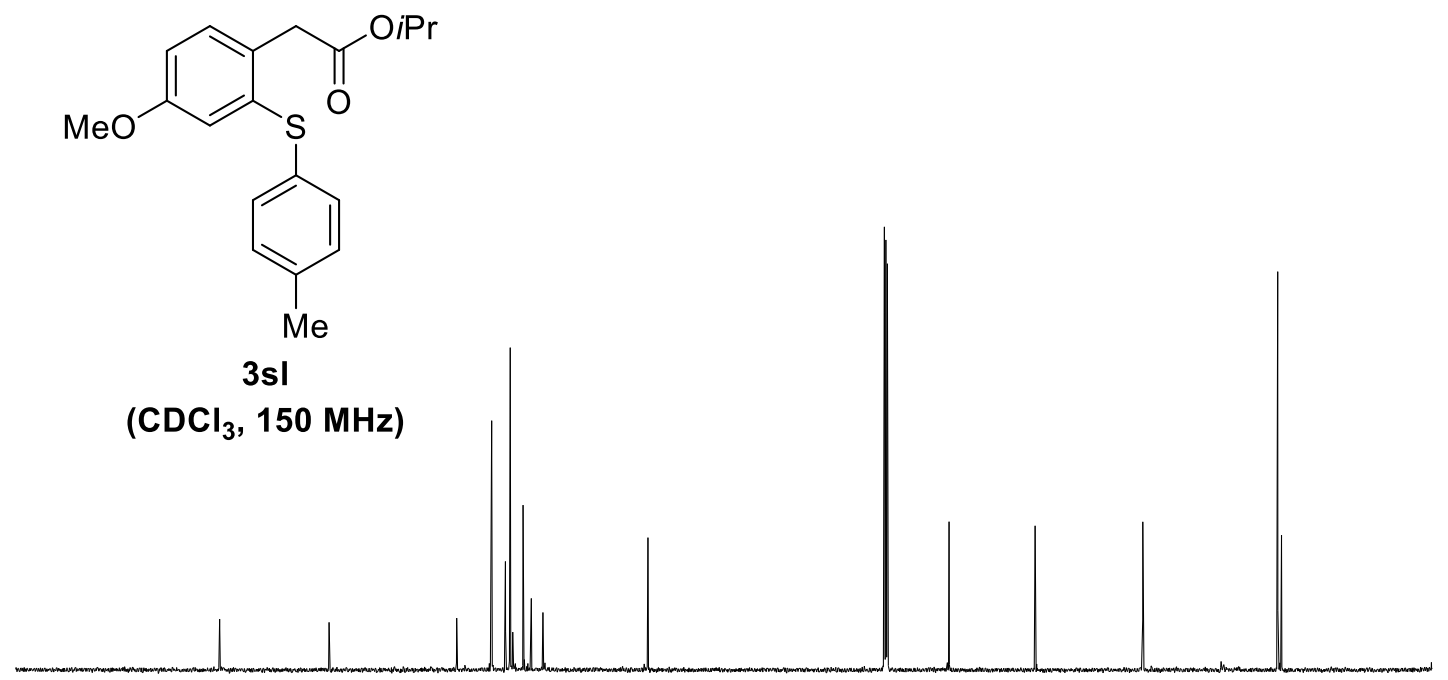

\begin{tabular}{lllllllllllllllllllll}
\hline 200 & 190 & 180 & 170 & 160 & 150 & 140 & 130 & 120 & 110 & 100 & 90 & 80 & 70 & 60 & 50 & 40 & 30 & 20 & 10 & 0 \\
\hline
\end{tabular} 
<smiles>CCCOC(=O)Cc1ccc(OC)cc1Sc1ccc(OC)cc1</smiles>

$3 \mathrm{sm}$

$\left(\mathrm{CDCl}_{3}, 600 \mathrm{MHz}\right)$

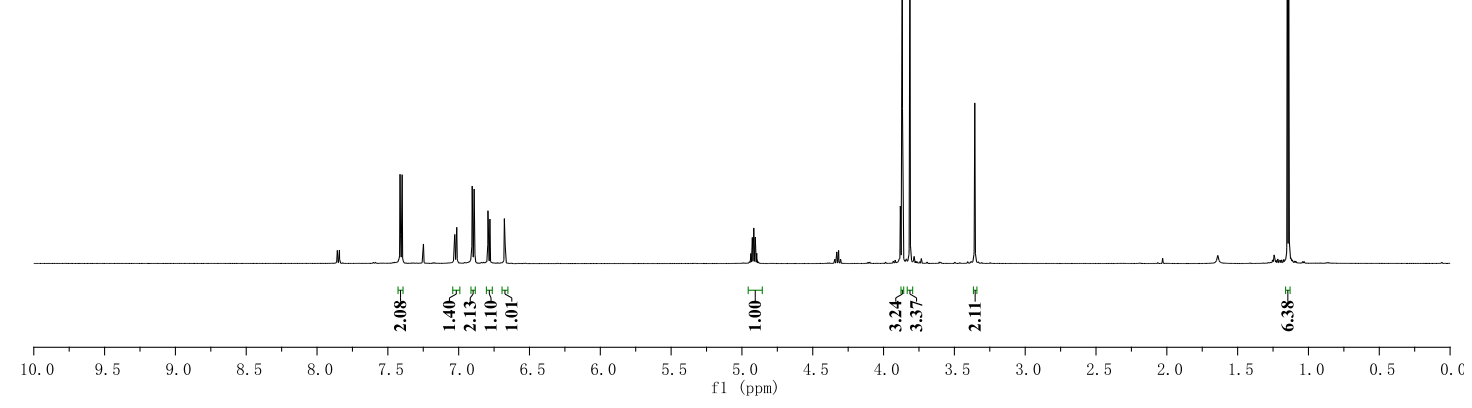

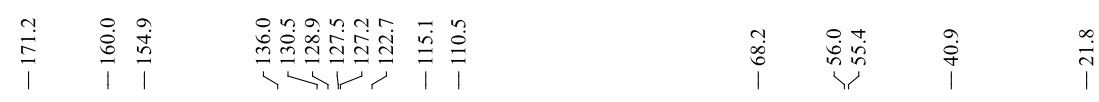<smiles>CCCOC(=O)Cc1ccc(OC)cc1Sc1ccc(OC)cc1</smiles>

$3 \mathrm{sm}$

$\left(\mathrm{CDCl}_{3}, 150 \mathrm{MHz}\right)$

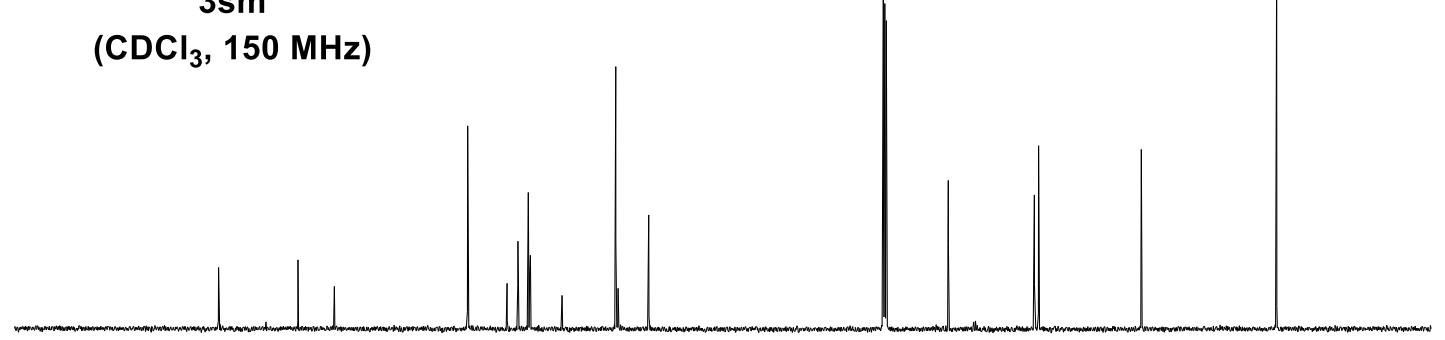

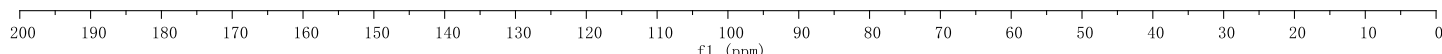




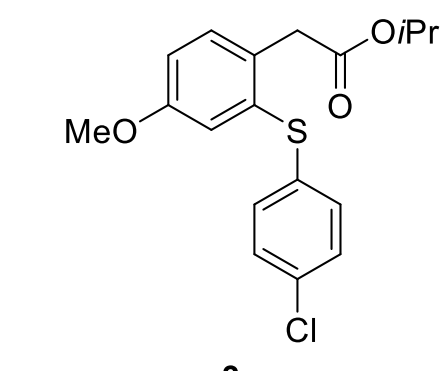

3sn

$\left(\mathrm{CDCl}_{3}, 600 \mathrm{MHz}\right)$

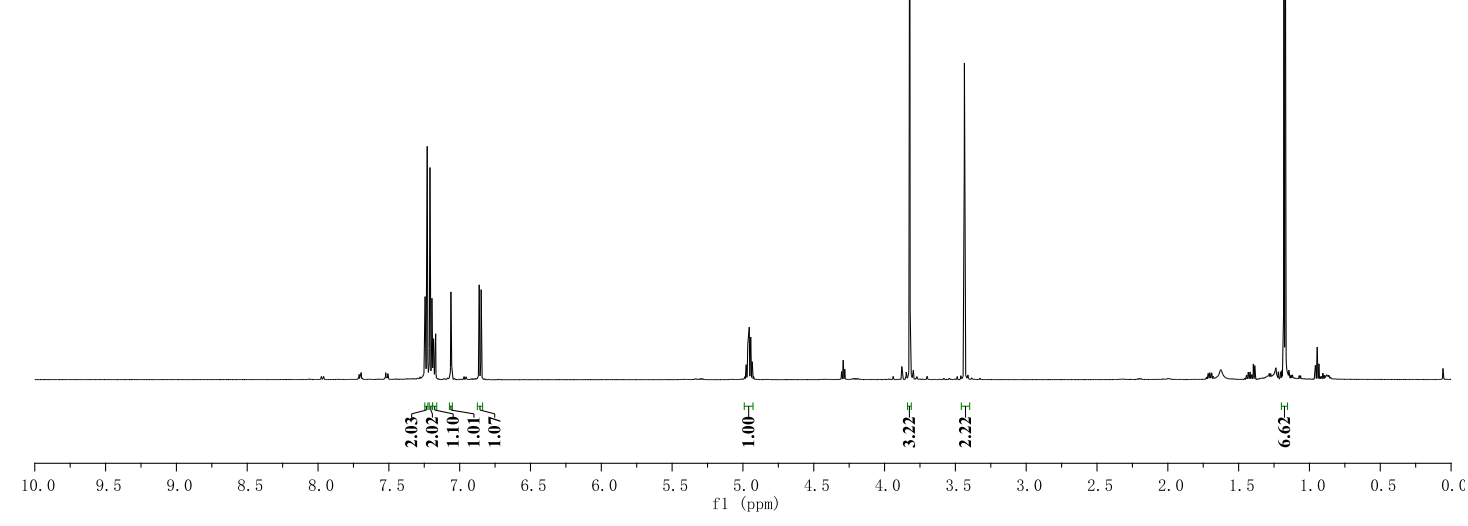

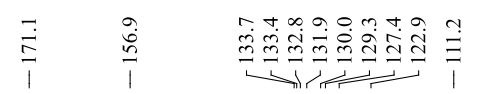

$\begin{array}{cccc}m & \overrightarrow{0} & \hat{+} & \stackrel{+}{i} \\ \substack{\infty \\ 0} & 1 & 1 & 1\end{array}$<smiles>COc1ccc(CC(=O)OC(C)C)c(Sc2ccc(Cl)cc2)c1</smiles>

3sn

$\left(\mathrm{CDCl}_{3}, 150 \mathrm{MHz}\right)$

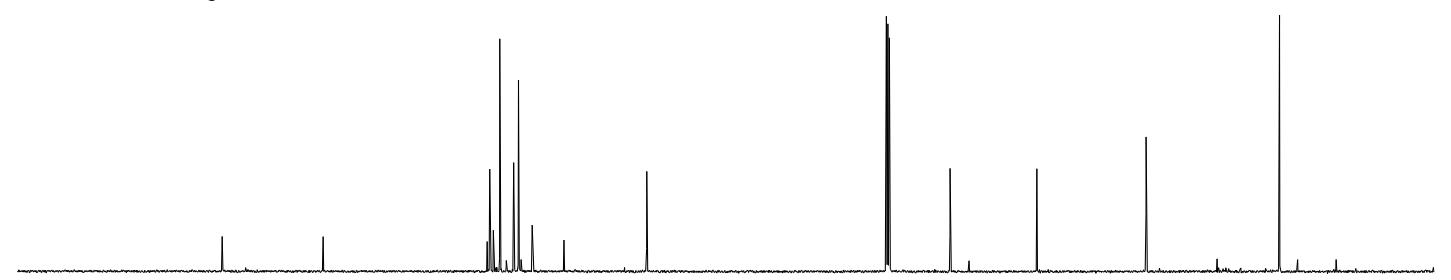

200

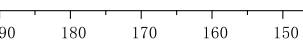

$140 \quad 130$

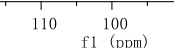


<smiles>Cc1ccc([Se]c2ccccc2)c(CC(=O)O)c1</smiles>

4

$\left(\mathrm{CDCl}_{3}, 600 \mathrm{MHz}\right)$
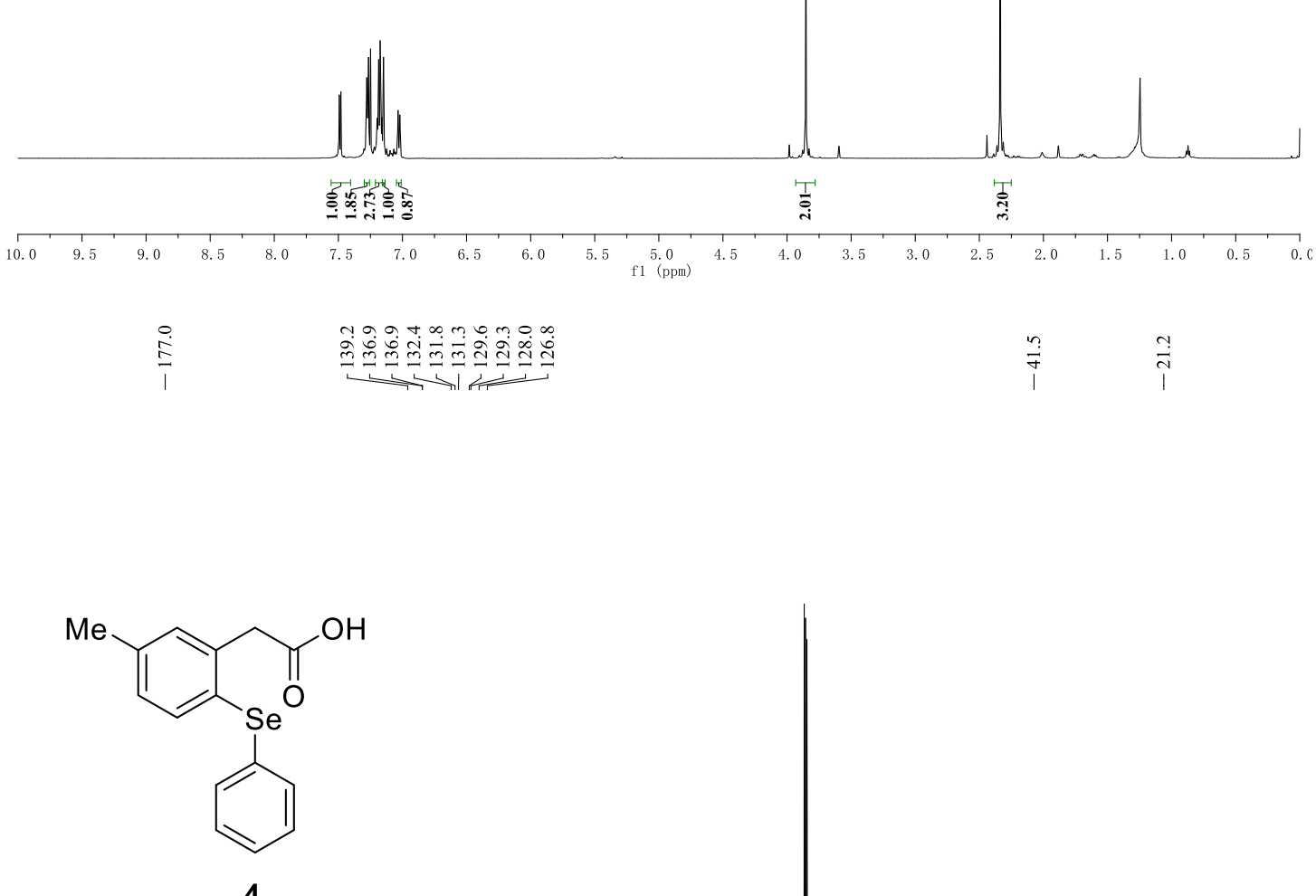

$\left(\mathrm{CDCl}_{3}, 600 \mathrm{MHz}\right)$

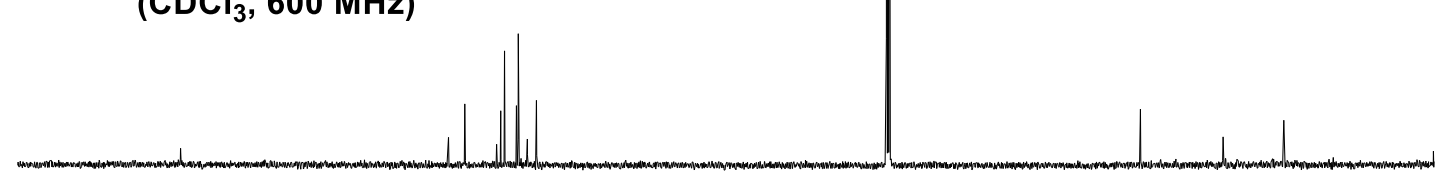

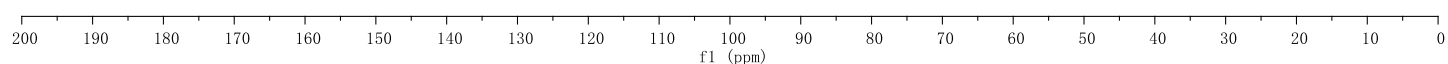


<smiles>Cc1ccc([Se](=O)(=O)c2ccccc2)c(CC(=O)NC(C)C)c1</smiles>

5

$\left(\mathrm{CDCl}_{3}, 600 \mathrm{MHz}\right)$

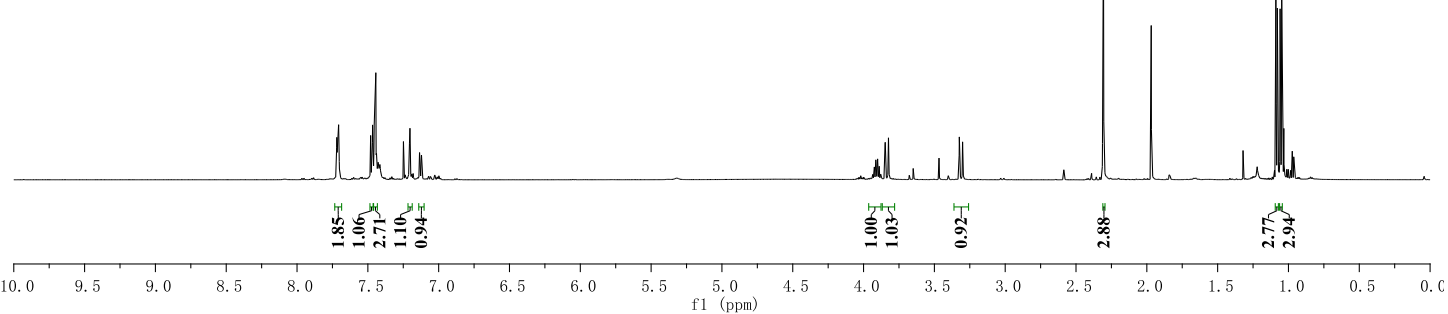

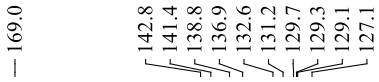

辛<smiles>Cc1ccc(S(=O)(=O)c2ccccc2)c(CC(=O)NC(C)C)c1</smiles>

5

$\left(\mathrm{CDCl}_{3}, 150 \mathrm{MHz}\right)$

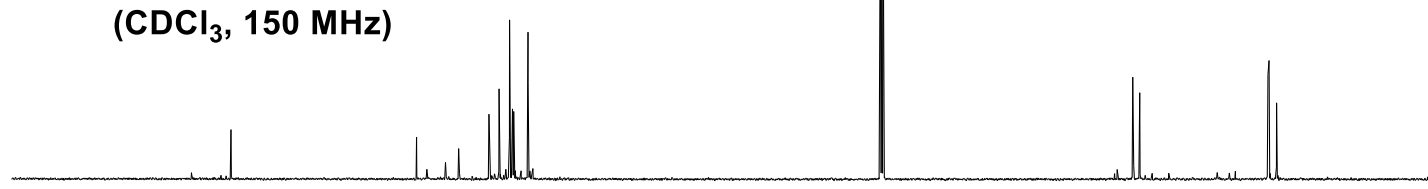

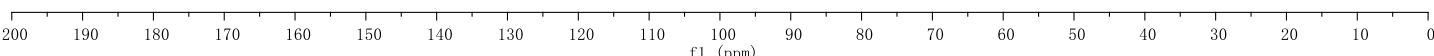




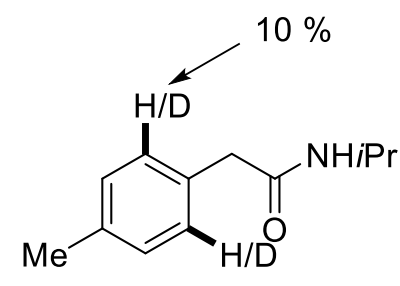

$[D]_{n}-1 a$

(CDCl3, $600 \mathrm{MHz}$ )

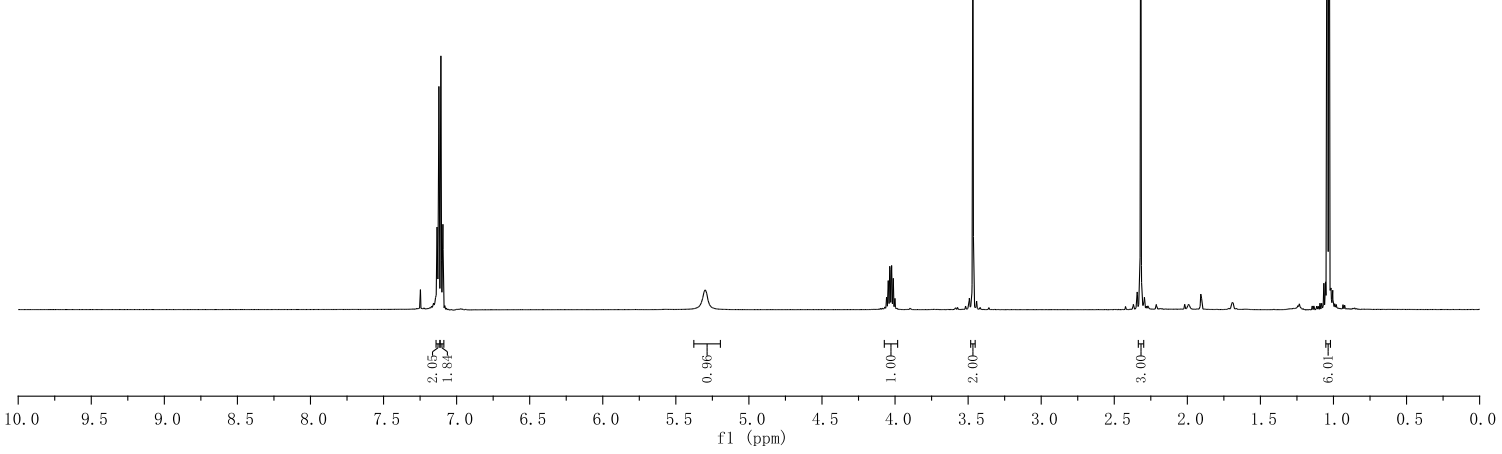

